\author{
UNIVERSIDADE DE SÃO PAULO \\ ESCOLA DE ARTES, CIÊNCIAS E HUMANIDADES \\ PROGRAMA DE PÓS-GRADUAÇÃO EM MODELAGEM DE SISTEMAS \\ COMPLEXOS
}

WAYNER DE SOUZA KLËN

Dinâmica relativística de partículas em torno de objetos ultracompactos

São Paulo

2019 


\section{Dinâmica relativística de partículas em torno de objetos ultracompactos}

Versão corrigida

Dissertação apresentada à Escola de Artes, Ciências e Humanidades da Universidade de São Paulo para obtenção do título de Mestre em Ciências pelo Programa de Pós-graduação em Modelagem de Sistemas Complexos.

Versão corrigida contendo as alterações solicitadas pela comissão julgadora em 29 de Julho de 2019. A versão original encontra-se em acervo reservado na Biblioteca da EACH/USP e na Biblioteca Digital de Teses e Dissertações da USP (BDTD), de acordo com a Resolução CoPGr 6018, de 13 de outubro de 2011.

Área de concentração: Sistemas Dinâmicos e Relatividade Geral

Orientador: Prof. Dr. Carlos Molina Mendes

São Paulo 
Autorizo a reprodução e divulgação total ou parcial deste trabalho, por qualquer meio convencional ou eletrônico, para fins de estudo e pesquisa, desde que citada a fonte.

CATALOGAÇÃO-NA-PUBLICAÇÃO

(Universidade de São Paulo. Escola de Artes, Ciências e Humanidades. Biblioteca) CRB 8 - 4936

Klën, Wayner de Souza

Dinâmica relativística de partículas em torno de objetos ultracompactos / Wayner de Souza Klën ; orientador, Carlos Molina Mendes - 2019.

$113 \mathrm{f}$. : il.

Dissertação (Mestrado em Ciências) - Programa de PósGraduação em Modelagem de Sistemas Complexos, Escola de Artes, Ciências e Humanidades, Universidade de São Paulo. Versão corrigida.

1. Sistemas dinâmicos. 2. Buracos negros. 3. Estabilidade de Lyapunov. I. Mendes, Carlos Molina. II. Título.

CDD 22.ed.- 530.131 
Dissertação de autoria de Wayner de Souza Klën, sob o título "Dinâmica relativística de partículas em torno de objetos ultracompactos", apresentada à Escola de Artes, Ciências e Humanidades da Universidade de São Paulo, para obtenção do título de Mestre em Ciências pelo Programa de Pós-graduação em Modelagem de Sistemas Complexos, na área de Sistemas Dinâmicos e Relatividade Geral, aprovada em 29 de Julho de 2019 pela comissão julgadora constituída pelos doutores:

Prof. Dr. Carlos Molina Mendes

Instituição: EACH - USP

Presidente

Prof. Dr. Alberto Vazquez Saa

Instituição: IMECC - UNICAMP

Prof. Dr. Iberê Luiz Caldas

Instituição: IF - USP

Prof. Dr. Masayuki Oka Hase

Instituição: EACH - USP 
Dedico este trabalho à minha Mãe, Maria Délza Duarte de Souza, e à todos de minha família. 


\section{AGRADECIMENTOS}

Gostaria de agradecer primeiramente a minha família, em especial à minha mãe Maria Délza, pessoa a quem tenho profunda admiração e amor, gostaria de agradecer ao meu irmão Wesley pelo companheirismo e paciência durante a minha formação. Deixo aqui o meu agradecimento a minha prima, Priscila Souza por ter me orientado e ensinado inúmeras coisas durante o meu período como docente. Gostaria de deixar expressa a minha gratidão ao meu orientador, o Prof. Dr. Carlos Molina Mendes, por sua paciência e dedicação no decorrer do mestrado, sempre ímpares. Agradeço ao Prof. Dr. Masayuki Oka Hase pelo curso de métodos matemáticos e pelas conversas sobre a vida acadêmica, bem como agradeço ao Prof. Dr. Fernando Fagundes Ferreira pelas intuições sobre o problema de pesquisa.

Um agradecimento especial para a Rayssa Viana, que tem sido uma grande amiga e companheira durante a minha formação, em especial nesta fase, meine liebe.

Aos companheiros de mestrado, em especial ao Matheus Moreira, com quem aprendi muito sobre programação. Gostaria de agradecer também ao companheiro Georges Stavrakas quem me auxilou com a sua dedicação em nos ensinar os fundamentos da linguagem Python.

Agradeço aos colegas do LACITE (Laboratório de Ciências da Terra) por sempre me receberem de braços abertos, obrigado pelos bons cafés e pelos momentos de descontração, em especial a Prof. ${ }^{a}$ Dr. ${ }^{a}$ Wânia Duleba por me proporcionar a oportunidade de conhecer o LNLS (Laboratório Nacional de Luz Síncrotron).

Por fim, gostaria de agradecer à Coordenação de Aperfeiçoamento de Pessoal de Nível Superior - CAPES, pelo apoio financeiro durante o meu mestrado, o que sem dúvida foi fundamental para a conclusão desta pesquisa. O presente trabalho foi realizado com apoio da Coordenação de Aperfeiçoamento de Pessoal de Nível Superior - Brasil (CAPES) - Código de Financiamento 001. 
"So very wise in their own eyes the world's great minds will one day find that for life they studied, worked, and pined but in wisdom made by man alone that a high IQ with low regard will be dethroned and from heaven barred." 


\section{RESUMO}

\section{KLËN, Wayner de Souza. Dinâmica relativística de partículas em torno de}

objetos ultracompactos. 2019. 113 f. Dissertação (Mestrado em Ciências) - Escola de Artes, Ciências e Humanidades, Universidade de São Paulo, São Paulo, 2019.

Nesta dissertação de mestrado o problema da estabilidade de geodésicas do tipo luz e do tipo tempo é estudado sobre o ponto de vista do formalismo de sistemas dinâmicos. Uma breve revisão bibliográfica sobre aspectos importantes de sistemas dinâmicos contínuos no tempo é realizada, bem como uma sucinta revisão de tópicos de interesse em relatividade geral. As equações de movimento para as geodésicas são deduzidas para geometrias com simetria esférica, e o caso Schwarzschild é inicialmente analisado. Em seguida, analisamos o caso das geometrias proposta por Casadio, Fabbri e Mazzacurati e um caso de buraco de minhoca assintoticamente de Sitter. A caracterização dos pontos fixos dos sistemas de interesse é feita, e a sua estabilidade é analisada sob a ótica dos métodos de Lyapunov e Jacobi, assim como bifurcações foram mapeadas. A fotosfera é caracterizada como um ciclo limite, sendo um ponto fixo estritamente instável no espaço de estados de buracos negros. A análise dos buracos de minhoca revelam a existência de uma fotosfera estável em determinadas regiões do espaço de parâmetros do sistema.

Palavras-chaves: Buracos Negros. Sistemas Dinâmicos. Estabilidade de Lyapunov. Estabilidade de Jacobi. Bifurcação Bogdanov-Takens. 


\begin{abstract}
KLËN, Wayner de Souza. Relativistic dynamics of particles around

ultracompact objects. 2019. 113 p. Dissertation (Master of Science) - School of Arts, Sciences and Humanities, University of São Paulo, São Paulo, 2019.

In this dissertation, the problem associated with the stability of timelike and null geodesics is studied from the dynamical system point of view. A succinct bibliographical review covering important aspects of time-continuous dynamical systems is made, and a short review about some topics of interest of general relativity is also presented. The geodesic equations of motion are shown for geometries with spherical symmetry, and the Schwarzschild case is first analyzed. In the following, we analyze the geometries proposed by Casadio, Fabbri, and Mazzacurati and an asymptotically de Sitter wormhole case. The characterization of the fixed points of the system is performed, and their stability is studied from the perspective of the Lyapunov and Jacobi methods, as well as the bifurcation analysis. The photon sphere is characterized as a limit cycle, being a strictly unstable fixed point in the state space of the system. The wormhole analysis reveals the existence of a stable photon sphere in certain regions of the parameter space of the system.
\end{abstract}

Keywords: Black Hole. Dynamical Systems. Lyapunov Stability. Jacobi Stability. BogdanovTakens Bifurcation. 


\section{LISTA DE FIGURAS}

Figura 1 - Possíveis tipos de órbitas entre pontos fixos. . . . . . . . . . . . . 25

Figura 2 - Curva inicial $S$ do espaço de configuração em linha contínua e região deslocada do espaço de configuração após a passagem de um $\delta t$. . . . 31

Figura 3 - Elemento infinitesimal de volume no espaço de configurações. . . . . . . 32

Figura 4 - Regiões da análise de estabilidade linear. . . . . . . . . . . . . . . . 40

Figura 5 - Esquema simbólico de um homeomorfismo $h: \mathbb{R}^{n} \mapsto \mathbb{R}^{n}$. . . . . . 41

Figura 6 - Diagrama completo da bifurcação Bogdanov-Takens. . . . . . . . . . . 44

Figura 7 - Campo de Killing associado à coordenada $\phi . . . . . . .50$

Figura 8 - Curvas para o potencial de Schwarzschild $V(r)$ associado à geodésicas do tipo tempo com vários valores de $L / M \ldots$. . . . . . . . . . . . 62

Figura 9 - Espaço de estados para o sistema com $L / M=4$ para diversos valores

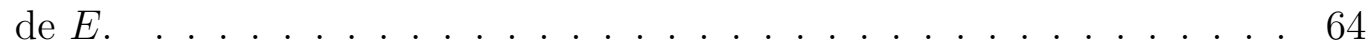

Figura 10 - Potenciais de Schwarzschild para geodésicas do tipo luz. . . . . . . . . 66

Figura 11 - Espaço de estados gerado via integral de linha convolucional do sistema definido por (167)-(168) . . . . . . . . . . . . . . . 68

Figura 12 - Trecho de órbita homoclínica (plano orbital) para partículas massivas com $L / M=12 \sqrt{3 / 35} \ldots \ldots \ldots \ldots \ldots$. . . . . . . . . . . . . . . . .

Figura 13 - Geodésicas nulas com $D=D_{c} \ldots \ldots \ldots \ldots \ldots$. . . . . . 73

Figura 14 - Coeficientes de Lyapunov no espaço de parâmetros do sistema. . . . . . 77

Figura 15 - Bifurcação fold do sistema com o parâmetros $\Lambda=3$ e $C=2 \times 10^{-4}$. . 78

Figura 16 - Gráfico do raio da garganta do buraco de minhoca em função do parâmetro $C \ldots \ldots \ldots$. . . . . . . . . . . . . . 81

Figura 17 - Órbitas radiais se aproximando e se afastando do buraco negro. A região em preto é a região do horizonte de eventos em $r=2 M$. . . . . . . 82

Figura 18 - Retrato de fase do sistema com $C=0.01, M=1, D_{c}=3 \sqrt{3} M$ e $E=1.84$ Figura 19 - Função $f_{\text {CFM }}(r)$ plotada para alguns valores de parâmetro de impacto $D .85$ Figura 20 - Possíveis casos a partir do parâmetro de bifurcação: (a) Parâmetro de impacto acima do valor crítico, (b) Parâmetro de impacto no valor crítico e (c) Parâmetro de impacto abaixo do valor crítico. . . . . . . . 86 
Figura 21 - Espaços de estados no plano orbital para diversos valores de $L$ e $\dot{r}$. A circunferência de preto representa a fotosfera em $r=3 M$ enquanto o

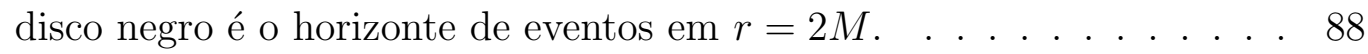

Figura 22 - Possíveis casos a partir do parâmetro de bifurcação $C$ : (a) Caso supercrítico $C=0$, (b) Caso supercrítico $C=-1$ (c) Caso crítico $C_{c}=-2$ e (d) Caso subcrítico $C=-3<C_{c} \ldots \ldots \ldots$. . . . . . . . . 90

Figura 23 - (a) Variedade de equilíbrio em função de $C$ com $D=3 \sqrt{3} M$, (b) variedade de equilíbrio do sistema em função de $D$ e (c) Variedade de equilíbrio em função de $C$ com $D=(C-4)^{3 / 2} M / 2 C^{1 / 2}$. . . . . . . . 91

Figura 24 - A esquerda, espaço de estados do sistema (270), a direita, espaço de estado do sistema (269) após a ocorrência da fold. . . . . . . . . . . . . 94

Figura 25 - Órbitas críticas de geodésica do tipo luz para $D_{c}=3 \sqrt{3} M$ : órbita homoclínica (esquerda) e região inacessível (direita). . . . . . . . . . 95

Figura 26 - Gráfico do potencial efetivo do buraco de minhoca CFM com $C=-0.1$ na figura de cima e $C=-2$ na figura de baixo. . . . . . . . . . . . 96 


\section{LISTA DE QUADROS}

Quadro 1 - Tabela da classificação dos pontos fixos segundo os autovalores associados para sistemas bidimensionais. . . . . . . . . . . . . . . . . 28

Quadro 2 - Quadro de comparação entre a estabilidade de Lyapunov e de Jacobi. 40

Quadro 3 - Possíveis casos para a solução CFM em termos do parâmetro $C$. . . . 60 


\section{LISTA DE TABELAS}

Tabela 1 - Coeficientes da forma normal da bifurcação Bogdanov-Takens. . . . . . 111 


\section{LISTA DE ABREVIATURAS E SIGLAS}

$\begin{array}{ll}\text { UCO } & \text { Objeto ultracompacto } \\ \text { EDO } & \text { Equação diferencial ordinária } \\ \text { RG } & \text { Relatividade geral } \\ \text { BN } & \text { Buraco negro } \\ \text { ISCO } & \text { Órbita circular estável mais interna } \\ \text { wh_dS } & \text { Buraco de minhoca assintoticamente de Sitter } \\ \text { CFM } & \text { Casadio-Fabbri-Mazzacurati } \\ \text { BBT } & \text { Bifurcação de Bogdanov-Takens }\end{array}$




\section{LISTA DE SÍMBOLOS}

\begin{tabular}{|c|c|}
\hline$\Lambda$ & Constante cosmológica \\
\hline$g_{\mu \nu}$ & Tensor métrico \\
\hline$T_{\mu \nu}$ & Tensor energia momento \\
\hline$G_{\mu \nu}$ & Tensor de Einstein \\
\hline$R_{\mu \nu}^{\sigma}$ & Tensor de Riemann-Christoffel \\
\hline$R_{\mu \nu}$ & Tensor de Ricci \\
\hline$\Gamma_{\mu \nu}^{\lambda}$ & Símbolo de Christoffel \\
\hline$\nabla_{\mu}$ & Derivada covariante \\
\hline $\mathcal{K}_{\mu}$ & Campo de Killing \\
\hline$\lambda$ & Parâmetro afim ao longo da geodésica \\
\hline$L$ & Momento angular \\
\hline$E$ & Energia total \\
\hline$\nu$ & Coeficiente de Lyapunov \\
\hline$M$ & Massa do corpo \\
\hline$D$ & Parâmetro de impacto \\
\hline$D_{c}$ & Parâmetro de impacto crítico \\
\hline$R$ & Escalar de Ricci \\
\hline$G$ & Constante gravitacional universal \\
\hline$c$ & Velocidade da luz no vácuo \\
\hline $\mathbb{R}$ & Conjunto dos números reais \\
\hline $\mathbb{C}$ & Conjunto dos números complexos \\
\hline$\Re(z)$ & Parte real do número complexo $z$ \\
\hline
\end{tabular}




$\begin{array}{ll}\Im(z) & \text { Parte imaginária do número complexo } z \\ \subset & \text { Subconjuto próprio } \\ \mathscr{L} & \text { Laplaciano } \\ \mathscr{H} & \text { Hamiltoniano } \\ \mathbf{J} & \text { Matriz Jacobiana } \\ \mathbf{H} & \text { Matriz Hessiana }\end{array}$




\section{SUMÁRIO}

Sistemas dinâmicos contínuos no tempo . . . . . . . . . . . . . . 22

Sistemas Hamiltonianos . . . . . . . . . . . . . . . . . . . . . . . 29

Buracos de minhoca assintoticamente de Sitter . . . . . . . . . . . 57 Limites extremo e quase-extremo da solução wh_dS . . . . . . . . . . 58 
4.6 Bifurcações do sistema e órbita homoclínica . . . . . . . . . . . 83

5 COMENTÁRIOS FINAIS . . . . . . . . . . 97

REFERENCIAS . . . . . . . . . . . . 99

APÊNDICE A - CÁLCULO DOS COEFICIENTES DA FORMA NORMAL DA BIFURCAÇÃO BOGDANOVTAKENS .................. 104

ÍNDICE REMISSIVO . . . . . . . . . . . . . . . 112 


\section{INTRODUÇÃO}

A relatividade geral tem se mostrado a teoria mais adequada para a descrição dos fenômenos gravitacionais em escala astrofísica. Ela é aplicada com sucesso numa vasta classe de situações, desde a descrição de estrelas isoladas, em distâncias típicas do sistema solar, até a cosmologia, que lida com as maiores distâncias possíveis de serem observadas. Em relatividade geral, a trajetória de partículas pontuais sob a ação exclusiva de um campo gravitacional é descrita por geodésicas em uma geometria pseudo-Riemaniana.

Para a obtenção de resultados analíticos envolvendo o tratamento da dinâmica geodésica, considerações de simplicidade são frequentemente assumidas. Por exemplo, a modelagem de objetos compactos é frequentemente feita através de soluções relativísticas esfericamente simétricas e estáticas. Espaços-tempos com estas simetrias são fenomenologicamente interessantes pois aproximam de forma razoável um grande conjunto de corpos astrofísicos reais. O foco deste trabalho será em espaços-tempos estáticos com simetria esférica. De fato, o estudo de geodésicas tipo tempo e tipo luz em torno em geometrias esféricas é tão antigo quanto o surgimento da relatividade geral. Dois dos testes clássicos da relatividade apresentados por Einstein em 1916 envolveram a análise de geodésicas na geometria de Schwarzschild. Especificamente, estes foram o cálculo relativístico da precessão do periélio da órbita de Mercúrio e a análise da deflexão da luz pelo Sol (EINSTEIN, 1916; WILL, 2014; DAVIDSON, 1920).

Mais recentemente, o estudo de geodésicas em geometrias não-triviais ganhou impulso com a introdução de técnicas de sistemas dinâmicos (CORNISH; LEVIN, 2003; LEVIN; O'REILLY; COPELAND, 2000; BELBRUNO; PRETORIUS, 2011; SHOOM, 2017; CARDOSO et al., 2009). Efetivamente, o problema geométrico do cálculo geodésico possui uma formulação Lagrangiana e Hamiltoniana, o que permite o uso do ferramental da análise dinâmica clássica. Assim, a tecnologia envolvendo sistemas dinâmicos é imediatamente aplicável ao problema.

A observação direta de ondas gravitacionais realizada em 2015 (ABBOTT; AL., 2016) tornou ainda mais importante a investigação de órbitas muito próximas de objetos ultracompactos, uma vez que a coalescência de objetos em buracos negros produz marcas distintas na radiação gravitacional emitida durante o processo (LEVIN; O'REILLY; COPELAND, 2000). O estudo da dinâmica geodésica em torno de objetos compactos pode 
tornar os dados obtidos nos detectores de ondas gravitacionais em importantes ferramentas na investigação astrofísica. Não obstante, os recentes avanços no tratamento computacional das imagens capturadas pelo projeto Event Horizon (COLLABORATION et al., 2019; AKIYAMA et al., 2019a; AKIYAMA et al., 2019b; AKIYAMA et al., 2019c; AKIYAMA et al., 2019d; AKIYAMA et al., 2019e) trazem uma nova luz à ótica geométrica associada aos objetos ultracompactos. Desta forma, o estudo teórico das trajetórias do tipo luz nestas geometrias se faz relevante uma vez que ele está relacionado com as sombras destes objetos, que por sua vez são propriedades observáveis do espaço-tempo.

A modelagem via o formalismo de sistemas dinâmicos recai sobre equações que descrevem a dinâmica do sistema, que em geral são de comportamento não linear, sendo a determinação de suas soluções uma tarefa complexa. Usualmente métodos numéricos são empregados. No entanto, existe uma série de questões referentes à previsibilidade fornecida por algoritmos numéricos, tornando necessário o desenvolvimento e emprego de outras técnicas de análise das soluções das equações do sistema.

É neste cenário que o estudo da teoria de sistemas dinâmicos se faz relevante, pois ela traz consigo técnicas para a análise e descrição das soluções de interesse. Uma das técnicas contidas na teoria de sistemas dinâmicos é a teoria qualitativa dos sistemas dinâmicos, que se ocupa da análise das propriedades topológicas das soluções das equações associadas à evolução dos sistemas, permitindo assim a previsão de propriedades dinâmicas como bifurcações ou eventualmente comportamentos periódicos. Putativamente, a modelagem de sistemas com muitos agentes envolvidos pode gerar comportamentos emergentes, isto é, comportamentos coletivos dos agentes que não tem relação direta com as propriedades individuais dos agentes. Sistemas com estas propriedades são usualmente chamados de sistemas complexos (SAYAMA, 2015; BOCCARA, 2010).

Nesta dissertação, estamos interessados na dinâmica relativística de partículas com massa de repouso nula se movimentando em torno de objetos ultracompactos no contexto de mundos brana. Estes objetos incluem estrelas de nêutrons ultracompactas, buracos negros e os ainda hipotéticos buracos de minhoca. A descrição das fotosferas, regiões de órbitas fechadas do tipo luz, em termos da linguagem de sistemas dinâmicos abrange algumas questões de especial interesse neste trabalho, tais como se existe a possibilidade de descrever as fotosferas como ciclos limite no plano orbital do sistema. A estabilidade da região da fotosfera via método de Lyapunov e Jacobi é de especial interesse neste trabalho, uma vez que convergência dos resultados de ambos os métodos pode ser interpretada como 
uma robustez do sistema (SABAU, 2005). Assim, a caracterização dinâmica das geodésicas do tipo luz para geometrias esfericamente simétricas em branas quadridimensionais é o tema central deste trabalho, sendo a análise de estabilidade avaliada por dois métodos essencialmente diferentes, bem como as possíveis bifurcações locais e globais decorrentes dos parâmetros geodésicos e da própria brana.

Uma das grandezas usualmente empregadas em sistemas dinâmicos para a avaliação da sensibilidade da dependência em relação às condições iniciais são os chamados coeficientes de Lyapunov. Atualmente, existem diversas formas de avaliar estas quantidades associadas à estabilidade de soluções, como, por exemplo, as apresentadas em Cardoso et al. (2009) e Cornish e Levin (2003). Por outro lado, métodos alternativos tem sido propostos para a avaliação de estabilidade de soluções como pode ser visto em Szydlowski (1993) e Eichhorn, Linz e Hänggi (2001). Neste trabalho, empregaremos os métodos diretos e indireto de Lyapunov para inferir sobre a estabilidade de soluções, que juntamente com a estabilidade de Jacobi nos fornecerá as informações necessárias para a estabilidade geodésica nas geometrias estudadas.

No que segue, esta dissertação está organizada conforme comentado a seguir. No capítulo 2 é apresentada uma revisão sobre sistemas dinâmicos, onde determinados conceitos e ferramentas usualmente empregadas na análise de sistemas dinâmicos são apresentados. No capítulo 3, uma breve revisão sobre relatividade geral é apresentada, onde as equações de campo de Einstein e as quantidades associadas são apresentadas, bem como as geometrias de interesse nesse trabalho. No capítulo 4, é conduzido um estudo sobre a descrição das equações que regem as geodésicas do tipo luz em termos do formalismo de sistema dinâmicos, estabilidade e bifurcações de pontos fixos são explorados e, finalmente, no capítulo 5 são apresentadas as conclusões e as perspectivas do trabalho. No apêndice A, é realizado o cálculo dos coeficientes da forma normal da bifurcação Bogdanov-Takens do sistema. 


\section{SISTEMAS DINÂMICOS}

Diversos fenômenos na natureza apresentam forte dependência temporal, tais como o crescimento populacional e o movimento de corpos celestes, de forma que a sua evolução pode ser descrita matematicamente por funções de um conjunto finito de variáveis dinâmicas $\mathbf{x}=\left(x_{1}, \ldots, x_{n}\right)^{T} \in \mathbb{R}^{n}$ (GREINER, 2003). A variedade de sistemas com essa propriedade são chamados de sistemas dinâmicos e podem ser definidos, de uma maneira um pouco menos formal, como sendo "sistemas dos quais os estados são unicamente especificados por um conjunto de variáveis e que o comportamento é descrito por regras predefinidas" (SAYAMA, 2015). No entanto, uma apresentação mais formal sobre o que é um sistema dinâmico pode ser conduzida. Discutiremos os elementos básicos deste formalismo neste capítulo.

\subsection{DEFINIÇÃO DE SISTEMAS DINÂMICOS}

Uma das possíveis definições de sistemas dinâmicos pode ser apresentada a partir da definição 2.1 (GIUNTI; MAZZOLA, 2012).

Definição 2.1 (Sistema dinâmico). Um sistema é dinâmico se, e somente se, for um tripla $\left(\mathcal{M}, g^{t}, T\right)_{t \in T}$ tal que,

- O $T$ está contido em $\mathbb{Z}, \mathbb{Z}^{+}, \mathbb{R}$ ou $\mathbb{R}^{+}$. Qualquer $t \in T$ é uma duração do sistema, e $T$ é chamado de seu conjunto temporal.

- O $\mathcal{M}$ é um conjunto não vazio e qualquer $x \in \mathcal{M}$ é chamado de estado do sistema e $\mathcal{M}$ é chamado de espaço de estados.

- $\left(g^{t}\right)_{t \in T}$ é a família de funções que levam de $\mathcal{M}$ em $\mathcal{M}$ indexadas por $T$, sendo que para qualquer $t \in T$, a função $g^{t}$ é chamada estado de transição de duração $t$, ou de forma mais econômica, transição-t.

- Para qualquer $v, t \in T$ e $x \in M$ sendo a condição inicial, valem

$$
\begin{aligned}
& -g^{0}(x)=x \\
& -g^{v+t}(x)=g^{v}\left(g^{t}(x)\right) .
\end{aligned}
$$

O primeiro item da definição 2.1 está relacionado com o tipo de domínio temporal do sistema dinâmico, uma vez que este pode ser contínuo (caso $T \in \mathbb{R}$ ) ou discreto (caso 
$T \in \mathbb{N}$ ) (MONTEIRO, 2006). O segundo item da definição 2.1 está relacionado com o conjunto de todas as possíveis configurações do sistema, sendo que um ponto $x \in \mathcal{M}$ descreve o estado atual do sistema, enquanto o conjunto $\mathcal{M}$ contém todas as possíveis configurações do sistema. O terceiro item da definição 2.1 diz respeito ao estado do sistema após uma duração de $t \in T$ dado um estado inicial do sistema $t_{0} \in T$ e o quarto item trata da regra de evolução do sistema, onde o primeiro subitem expressa que para qualquer estado do sistema, uma evolução de duração 0 não altera o estado do sistema, e o segundo subitem nos diz que qualquer evolução de duração $v+t$ pode ser decomposta em duas evoluções consecutivas com aplicações sucessivas de $g$.

\subsection{SISTEMAS DINÂMICOS CONTÍNUOS NO TEMPO}

Inúmeros sistemas presentes na natureza podem ser modelados através de sistemas dinâmicos de tempo contínuo, desde dinâmicas populacionais até mesmo a dinâmica de indivíduos "racionais" em processo de tomada de decisão em uma negociação (SAYAMA, 2015). Para que o sistema seja de tempo contínuo é necessário que o domínio temporal esteja contido na reta real, ou seja, $t \in \mathbb{R}$ e sua evolução deve ser governada por uma ou mais equações integrais (equações que contêm funções operadas por integrais) ou por equação diferenciais (equações que são expressas em termos de derivadas de uma variável desconhecida $x \in \mathbb{R}$ ), na forma (GREINER, 2003)

$$
\frac{\mathrm{d} \mathbf{x}(t)}{\mathrm{d} t}=\mathbf{F}(\mathbf{x}(t), t ; \mu)
$$

onde $\mathbf{F}$ pode ser uma função não linear das coordenadas $\mathbf{x}$ e pode depender explicitamente do tempo (caso $\mathbf{F}$ não dependa explicitamente do tempo o sistema é chamado de autônomo). O valor $\mu$ está associado aos parâmetros de controle do sistema. ${ }^{1}$ A definição formal de sistemas dinâmicos contínuos no tempo é dada na definição 2.1 identificando o domínio temporal como a reta real (HIRSCH; SMALE; DEVANEY, 2012).

\subsection{ESPAÇO DE ESTADOS}

Em geral, a análise da teoria qualitativa de sistemas dinâmicos é realizada com o auxílio do chamado espaço de estados. O espaço de estados associado ao sistema dinâmico

\footnotetext{
1 Parâmetros são valores que influenciam diretamente no sistema mas não dependem das coordenadas $\mathbf{x}$.
} 
(1) contém informações relevantes sobre o comportamento de suas soluções, mesmo sem a realização da integração do sistema.

Dizemos que o espaço de estados é um espaço $n$-dimensional, no qual estão contidas todas as possíveis configurações do sistema que, para o caso contínuo é uma variedade (MONTEIRO, 2006). No contexto deste trabalho entende-se que uma $n$-variedade é um espaço topológico que localmente se comporta como um espaço Euclidiano em cada um de seus pontos. Segundo esta definição, os pontos contidos na $n$-variedade possuem uma vizinhança que é homeomórfica a uma superfície Euclidinana de dimensão $n$ (LEE, 2010).

Um estado qualquer deste sistema é representado por um ponto no espaço de estados $\left\{x_{1}(t), x_{2}(t), \ldots, x_{n}(t)\right\}$, sendo que com o decorrer do tempo este ponto de desloca formando uma trajetória que é governada pelo sistema de equações diferenciais (1). Pode-se dizer que o espaço de estados é o conjunto de todas as possíveis configurações do sistema (VULPIANI, 2010).

Dentro do espaço de estados, é possível identificar trajetórias realizadas pelas possíveis soluções do sistema, trajetórias estas que dependerão das condições iniciais (GIUNTI; MAZZOLA, 2012). O teorema de Picard-Lindelöf assegura a existência e unicidade destas trajetórias dado um conjunto de equações diferenciais associadas a uma condição inicial que satisfazem determinadas condições de continuidade ${ }^{2}$. Uma solução estacionária no espaço de estados, isto é, um ponto que não se desloca no espaço de estados, é chamado de ponto fixo.

Definição 2.2 (Ponto fixo). Um ponto fixo $\mathrm{x}_{0}$ no espaço de estados $\mathcal{M}$ do sistema dinâmico (1) é um ponto que satisfaz a seguinte condição,

$$
\mathbf{F}\left(\mathbf{x}_{0}, t ; \mu\right)=0
$$

Uma maneira de visualizar qualitativamente o comportamento do sistema é através da construção das curvas isóclinas. A existência de curvas isóclinas auxilia na construção do espaço de estados do sistema, pois ao longo destas curvas todas as inclinações da reta tangente a solução são iguais entre si.

Definição 2.3 (Curvas isóclinas). Seja um sistema dinâmico em $\mathbb{R}^{2}$ definido por EDOs contínuas $\dot{x}_{1}(t)$ e $\dot{x}_{2}(t)$, onde o ponto indica diferenciação em relação ao tempo. Uma $\overline{2}$ A saber, o sistema deve ser contínuo em $t$ e Lipschitz contínua em $\mathbf{x}(t)$ (DEVANEY, 2004). 
isóclina é uma curva no espaço de estados no qual as trajetórias tem o gradiente constante. Encontram-se as isóclinas por eliminação do parâmetro $t$, e tomando $x_{1}$ como a variável independente, assim para $x_{1}(t) \neq 0$,

$$
\frac{\mathrm{d} x_{2}}{\mathrm{~d} x_{1}}=\frac{\dot{x}_{2}}{\dot{x}_{1}}=\frac{F_{2}\left(x_{1}, x_{2}\right)}{F_{1}\left(x_{1}, x_{2}\right)}=k \in \mathbb{R} .
$$

A quantidade $k$ é a inclinação da curva integral $x_{2}\left(x_{1}\right)$ no plano $x_{1} \times x_{2}$, onde não há dependência temporal explícita.

Definição 2.4 (Órbita). Define-se órbita de $\mathbf{x}$ a trajetória orb $(\mathbf{x})=\left\{\mathbf{z}: \mathbf{z}=g^{t}(\mathbf{x})\right.$, para algum $t \in T\}$ e $\mathbf{x} \in \mathcal{M}$

As órbitas podem ser classificadas conforme o seu comportamento, ou seja, se elas conectam nenhuma, uma ou duas soluções de equilíbrio. As órbitas que conectam as soluções de equilíbrio possuem grande importância na caracterização da estabilidade estrutural do sistema dinâmico, estas órbitas podem ser classificadas como heteroclínicas ou homoclínicas, de acordo com as definições 2.5 e 2.6 a seguir.

Definição 2.5 (Órbita heteroclínica). É dito que uma solução x $(t)$ da equação (1) é uma órbita heteroclínica se a seguinte condição for satisfeita,

$$
\lim _{t \rightarrow \pm \infty} \mathbf{x}(t)=p_{ \pm}
$$

para os pontos fixos $p_{ \pm} \in \mathbb{R}^{n}$ (HOMBURG; SANDSTEDE, 2010).

Definição 2.6 (Órbita homoclínica). Uma solução $\mathbf{x}(t)$ é dita homoclínica se a condição da definição 2.5 for verdadeira com $p_{-}=p_{+}$(HOMBURG; SANDSTEDE, 2010).

Em geral, órbitas homoclínicas são trajetórias que são, ao mesmo tempo, parte da variedade estável e instável de um ponto fixo, enquanto uma órbita heteroclínica é uma trajetória que se encontra na variedade estável de um ponto fixo e variedade instável de outro ponto fixo (HOMBURG; SANDSTEDE, 2010).

Uma situação que pode ocorrer no estudo de sistemas dinâmicos é a criação ou aniquilação de pontos fixos, bem como a geração ou aniquilação de órbitas homoclínicas e heteroclínicas. Quando esses fenômenos ocorrem, em geral, temos associado a eles uma bifurcação. Dado um sistema dinâmico na forma (1), uma bifurcação é uma mudança de 
comportamento qualitativo do sistema conforme se varia os parâmetros do conjunto de parâmetros do sistema $\mu$ (BERNARDO et al., 2008).
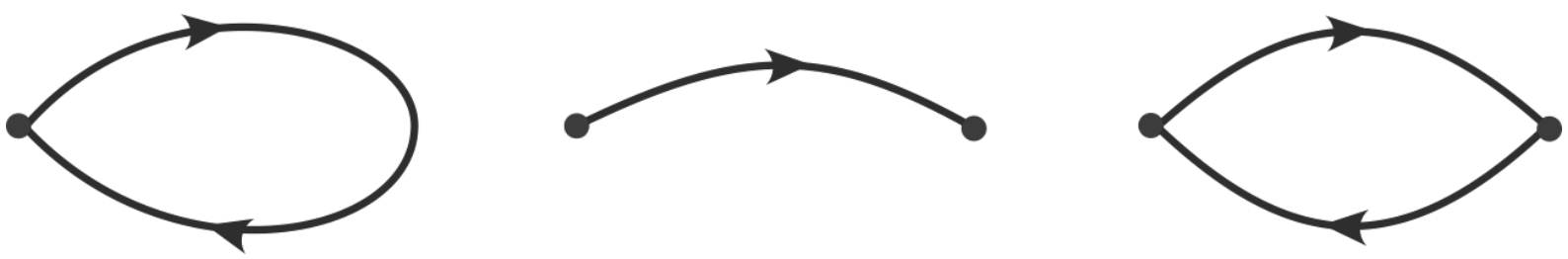

Figura 1 - Possíveis tipos de órbitas entre pontos fixos.

Fonte: Homburg e Sandstede (2010).

Na figura 1, a órbita da esquerda é uma órbita homoclínica, pois está contida na variedade estável e instável do ponto fixo, formando um loop fechado no próprio ponto, enquanto a órbita do centro representa uma órbita heteroclínica simples, e a última órbita à direta representa um ciclo heteroclínico entre dois pontos fixos.

\subsection{ATRATORES E REPULSORES}

Dado um sistema dinâmico, é possível observar determinadas estruturas em seu espaço de estados. Podem existir determinadas condições iniciais que tendem a uma determinada região do espaço de estados conforme se evolui o sistema, enquanto existem outras condições iniciais que se afastam de certas regiões do sistema. Desta forma, este comportamento classifica determinadas regiões do espaço de estados como segue.

Definição 2.7 (Atrator). Um atrator no espaço de estados $\mathcal{M}$ de um sistema dinâmico é um subconjunto $\mathcal{A} \subset \mathcal{M}$ tal que satisfaça as seguintes condições (MONTEIRO, 2006),

- $\mathcal{A}$ é um conjunto invariante, isto é, qualquer trajetória $\mathbf{x}(t)$ iniciada em $\mathcal{A}$ permanece em $\mathcal{A} \forall t \in T$;

- $\mathcal{A}$ atrai um conjunto aberto de condições iniciais, isto é, existe um hipervolume $\mathcal{B}$ que contém $\mathcal{A}$, tal que para qualquer condição inicial $\mathbf{x}_{0}=\mathbf{x}(0) \in \mathcal{B}$ a distância ${ }^{3}$ entre a trajetória $\mathbf{x}(t)$ e $\mathcal{A}$ tende a zero conforme $t \rightarrow \infty$;

- $\mathcal{A}$ é mínimo, ou seja, não existe subconjunto $s \subset \mathcal{A}$ que satisfaça as duas condições anteriores.

3 Isto é, para qualquer vizinhança $N \in \mathcal{B}$ existe uma constante positiva $T$ tal que $x(t) \in N$ para todo real $t>T$. 
Uma possibilidade alternativa é a existência de órbitas que tendem a se afastar do ponto de determinadas regiões do espaço de estados, caracterizando a repulsão em relação ao ponto fixo.

Definição 2.8 (Repulsor). Um repulsor no espaço de estados $\mathcal{M}$ de um sistema dinâmico é um subconjunto $\mathcal{U} \subset \mathcal{M}$ tal que satisfaça as seguintes condições (MONTEIRO, 2006),

- $\mathcal{U}$ é um conjunto invariante, isto é, qualquer trajetória $\mathbf{x}(t)$ iniciada em $\mathcal{U}$ permanece em $\mathcal{U} \forall t \in T$;

- $\mathcal{U}$ afasta um conjunto aberto de condições iniciais, isto é, existe um hipervolume $\mathcal{C}$ que contém $\mathcal{U}$, tal que para qualquer condição inicial $\mathbf{x}_{0}=\mathbf{x}(0) \in \mathcal{C}$ a distância entre a trajetória $\mathbf{x}(t)$ e $\mathcal{U}$ tende a zero conforme $t \rightarrow-\infty$;

- $\mathcal{U}$ é mínimo, ou seja, não existe subconjunto $s \subset \mathcal{U}$ que satisfaça as duas condições anteriores.

Ao colecionar os pontos no entorno destas estruturas, podemos definir regiões específicas do espaço de estados como segue,

Definição 2.9 (Bacia de Atração). Dado um sistema dinâmico, o subconjunto de condições iniciais $S$ do espaço de estados $\mathcal{M}$ que levam ao atrator $\mathcal{A}$ conforme $t \rightarrow \infty$ é chamado de bacia de atração do atrator $\mathcal{A}$ (MONTEIRO, 2006).

Definição 2.10 (Bacia de repulsão). Dado um sistema dinâmico, o subconjunto de condições iniciais $U$ do espaço de estados $\mathcal{M}$ que levam ao repulsor $\mathcal{U}$ conforme $t \rightarrow-\infty$ é chamado de bacia de repulsão.

Atratores e repulsores podem ser, por exemplo, pontos fixos isolados ou agrupados, ou ainda ciclos limites. Por vezes, um mesmo ponto fixo pode possuir regiões de repulsão e atração simultaneamente, como, por exemplo, em pontos fixos do tipo sela, como veremos na classificação dos pontos fixos.

Um tipo alternativo de solução de equilíbrio que pode ser encontrada em um sistema dinâmico é o chamado ciclo limite. A definição de ciclo limite é dada na definição 2.12 (LORENZ, 1963).

Definição 2.11 (Ciclo ou centro). Dado um sistema dinâmico, um ciclo é uma solução $\gamma\left(x_{0}, t\right)$ descrita por uma curva fechada no espaço de estados (MONTEIRO, 2006; VULPIANI, 2010). 
Um ciclo limite é uma trajetória fechada e isolada no espaço de configuração do sistema, ou seja, uma trajetória fechada que não possui outras trajetórias fechadas infinitesimalmente próximas a ela (MONTEIRO, 2006). O ciclo limite pode ter um comportamento atrativo, repulsivo ou até mesmo parcialmente ambos quando tomadas soluções em sua periferia. Então seguem as definições (LORENZ, 1963).

Definição 2.12 (Ciclo limite assintoticamente estável). Se todas as trajetórias $\gamma\left(x_{0}, t\right)$ que se iniciam em uma periferia do ciclo convergem para o ciclo quando $t \rightarrow \infty$, então este ciclo é dito um ciclo limite assimptoticamente estável (MONTEIRO, 2006; STROGATZ, 2018).

Definição 2.13 (Ciclo limite assintoticamente instável). Se todas as trajetórias $\gamma\left(x_{0}, t\right)$ que se iniciam em uma periferia do ciclo divergem do ciclo quando $t \rightarrow \infty$, então este ciclo é dito um ciclo limite assimptoticamente instável (MONTEIRO, 2006).

Definição 2.14 (Ciclo limite semi-estável). Se algumas trajetórias $\gamma_{\beta}\left(x_{0}, t\right)$ que se iniciam em uma periferia do ciclo limite convergem para o ciclo, e outras $\gamma_{\alpha}\left(x_{0}, t\right)$ divergem do ciclo, este é chamado de ciclo limite semi-estável (MONTEIRO, 2006; STROGATZ, 2018).

Um critério importante para a existência de ciclos limites é o chamado critério do ponto fixo (MONTEIRO, 2006).

Teorema 2.15 (Critério do ponto fixo). Uma trajetória fechada no espaço de estados de um sistema dinâmico contínuo possui um ponto fixo em seu interior.

Assim, uma região que é simplesmente conexa (sem buracos) e não possui ao menos um ponto fixo em seu interior não pode conter ciclos limite, pois não haverão órbitas fechadas nesta região.

Uma ferramenta alternativa para a eliminação da existência de ciclo limites em sistemas dinâmicos suaves é mostrar que o sistema não admite órbitas fechadas. Esta tarefa pode ser realizada através do critério de Bendixson introduzido no teorema 2.16.

Teorema 2.16 (Critério de Bendixson). Se $\frac{\partial f(x, y)}{\partial x}$ e $\frac{\partial g(x, y)}{\partial y}$ são contínuas em uma região $R$ a qual é simplesmente conexa, $e$

$$
\frac{\partial f}{\partial x}+\frac{\partial g}{\partial y} \neq 0 \quad \text { em todo ponto de } R
$$


então o sistema

$$
\begin{aligned}
& x^{\prime}=f(x, y) \\
& y^{\prime}=g(x, y)
\end{aligned}
$$

não possui trajetórias fechadas dentro de $R$, e consequentemente não possui ciclos limite (MONTEIRO, 2006).

O critério de Bendixson afirma que a existência de um ciclo limite está condicionada à existência de ao menos uma órbita fechada, caso esta órbita fechada não exista a eliminação da existência de um ciclo limite é direta.

\subsection{CLASSIFICAÇÃO TOPOLÓGICA DOS PONTOS FIXOS}

Dado um sistema dinâmico de tempo contínuo cuja a evolução é governada por uma equação diferencial ordinária, é possível estudar propriedades relevantes do sistema sem necessidade de se efetuar o cálculo analítico das possíveis soluções do sistema. Isto pode ser feito à luz da teoria qualitativa, que investiga características relevantes quando conduzida um análise sobre o espaço de estados. Um importante aspecto dessa análise são os chamados pontos fixos.

Os pontos fixos são pontos do espaço de estados onde a taxa de variação da solução do sistema é nula, ou seja, pontos de onde é possível extrair informações do comportamento assintótico do sistema em decorrência da independência temporal destes pontos. Os pontos fixos podem ser classificados a partir dos autovalores do sistema linearizado avaliados no ponto. Neste trabalho os sistemas de interesse serão analisados inicialmente em duas

\begin{tabular}{|c|c|c|}
\hline Tipo de ponto & Autovalores & Classificação topológica \\
\hline \multirow{5}{*}{ Hiperbólico } & 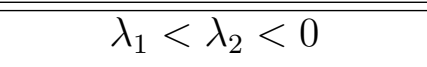 & Nó estável \\
\hline & $\nu_{1}>\nu_{2}>0$ & Nó instável \\
\hline & $\nu_{1}<0<\nu_{2}$ & Ponto fixo de sela \\
\hline & $\nu_{1,2}=\mu \pm i \omega \& \mu<0$ & Ponto espiral estável \\
\hline & $\nu_{1,2}=\mu \pm i \omega \& \mu>0$ & $\begin{array}{c}\text { Ponto espiral instável } \\
\text { (espiral repulsora) }\end{array}$ \\
\hline Não Hiperbólico & $\nu_{1,2}=\mu \pm i \omega \& \mu=0$ & $\begin{array}{l}\text { Ponto fixo elíptico } \\
\text { (centro) }\end{array}$ \\
\hline
\end{tabular}
dimensões, assim, os possíveis casos de pontos fixos estão apresentados na quadro 1.

Quadro 1 - Tabela da classificação dos pontos fixos segundo os autovalores associados para sistemas bidimensionais. 
Quando é necessário realizar a análise em torno de um ponto fixo de um sistema não linear, em geral verifica-se se este ponto é hiperbólico ${ }^{4}$ segundo a definição 2.17.

Definição 2.17 (Ponto fixo hiperbólico). Seja x um ponto fixo no espaço de estados do sistema e $\nu_{ \pm} \in \mathbb{C}$ os autovalores associados a este ponto. É dito que $\mathbf{x}$ é um ponto fixo hiperbólico se $\Re\left(\nu_{ \pm}\right) \neq 0$ (VULPIANI, 2010).

Caso a definição 2.17 seja satisfeita, pode-se utilizar o teorema de HartmanGrobman. O teorema de Hartman-Grobman afirma que a dinâmica na variedade não linear é semelhante àquela da variedade linearizada na vizinhança de um ponto fixo hiperbólico (ABRAHAM et al., 1980; MONTEIRO, 2006). A estabilidade de um ponto de equilíbrio hiperbólico é preservada ao realizar uma linearização do sistema, fato que faz do espaço de estados topologicamente e orbitalmente equivalentes na vizinhança do ponto. Quando o ponto não é hiperbólico, a linearização não permite inferir sobre sua estabilidade (MONTEIRO, 2006).

No entanto, a análise via linearização fornece informações sobre o comportamento assintótico do ponto fixo, não sendo o suficiente para fornecer informações sobre toda uma região de comportamento transiente do sistema. Para obter informações sobre estas regiões é necessário o emprego de outras técnicas de análise.

\subsection{SISTEMAS HAMILTONIANOS}

Um subconjunto dos sistemas dinâmicos são os chamados sistemas Hamiltonianos, que constituem uma classe de sistemas dinâmicos conservativos. Os sistemas Hamiltonianos são construídos a partir de equações dinâmicas, chamadas equações de Hamilton, que por sua vez podem ser expressas em termos de uma Lagrangiana. Para definir a Lagrangiana é necessário definir o chamado princípio de Hamilton, como na definição 2.18.

Definição 2.18. Seja um sistema mecânico. Os movimentos do sistema mecânico coincidem com os extremos do funcional (ARNOL'D, 2013),

$$
S[\mathbf{q}, \dot{\mathbf{q}}]=\int_{t_{0}}^{t} \mathscr{L} \mathrm{d} t,
$$

com

$$
\mathscr{L}=T(\dot{\mathbf{q}})-U(\mathbf{q})
$$

4 Também chamado de singularidade elementar na literatura de sistemas dinâmicos (ABRAHAM et al., 1980). 
A definição 2.18 pode ser visualizada assumindo que $U=U(\mathbf{r})$ e $T=T(\dot{\mathbf{r}})=$ $\frac{1}{2} \sum m_{i} \dot{\mathbf{r}}_{i}^{2}$, e que derivando $\mathscr{L}$ com relação a $\mathbf{r}$ e $\dot{\mathbf{r}}$ respectivamente, se obtém

$$
\frac{\partial \mathscr{L}}{\partial \mathbf{r}}=-\frac{\partial U}{\partial \mathbf{r}} \equiv F(\mathbf{r})
$$

e

$$
\frac{\partial \mathscr{L}}{\partial \dot{\mathbf{r}}}=\frac{\partial T}{\partial \dot{\mathbf{r}}}=\sum_{i=1}^{n} m_{i} \dot{\mathbf{r}} \equiv \mathbf{p} .
$$

Uma consequência da definição acima é que qualquer que seja o sistema de coordenadas $\left(q_{1}, q_{2}, \ldots, q_{n}\right)$ no espaço de fase de $n$ massas pontuais, a evolução $\mathbf{q}$ com o tempo é governada pelas equações de Euler-Lagrange (ARNOL'D, 2013),

$$
\frac{\mathrm{d}}{\mathrm{d} t}\left(\frac{\partial \mathscr{L}}{\partial \dot{\mathbf{q}}}\right)-\frac{\partial \mathscr{L}}{\partial \mathbf{q}}=0
$$

Utilizando a terminologia de mecânica, é possível definir a função (8) como a Lagrangiana do sistema, onde $q_{i}$ são as coordenadas generalizadas, $\dot{q}_{i}$ são as velocidades generalizadas, $\partial \mathscr{L} / \partial \dot{q}_{i}$ são os momentos generalizados, $-\partial \mathscr{L} / \partial q_{i}$ são as forças generalizadas, $S$ é a ação e, por fim, as equações (11) são as equações de Euler-Lagrange.

As equações de Lagrange possuem um equivalente chamado de equações de Hamilton. Segue a definição de Hamiltoniano.

Definição 2.19 (Equações de Hamilton). Seja $\mathscr{H}: \mathbb{R}^{2 n} \rightarrow \mathbb{R}$ uma função de classe $C^{2}$, dita um Hamiltoniano e as coordenadas de $\mathbb{R}^{2 n}$ escritas na forma $(q, p)$, onde $q=\left(q_{1}, \ldots, q_{n}\right)$ e $p=\left(p_{1}, \ldots, p_{n}\right)$. É dito que o sistema de equações

$$
\begin{gathered}
-\frac{\mathrm{d} p_{j}}{\mathrm{~d} t}=\frac{\partial \mathscr{H}}{\partial q_{j}}, \\
\frac{\mathrm{d} q_{j}}{\mathrm{~d} t}=\frac{\partial \mathscr{H}}{\partial p_{j}},
\end{gathered}
$$

com $j=1, \ldots, n$, é Hamiltoniano com $n$ graus de liberdade (BARREIRA; VALLS, 2012).

A função $\mathscr{H}$ pode ser obtida da Lagrangiana (8), fornecendo a seguinte expressão (GREINER, 2003),

$$
-\frac{\partial \mathscr{H}}{\partial t}=\frac{\partial \mathscr{L}}{\partial t}
$$

Estes sistemas constituem uma importante classe sistemas dinâmicos, os chamados sistemas Hamiltonianos. No contexto da mecânica Hamiltoniana é possível construir um 


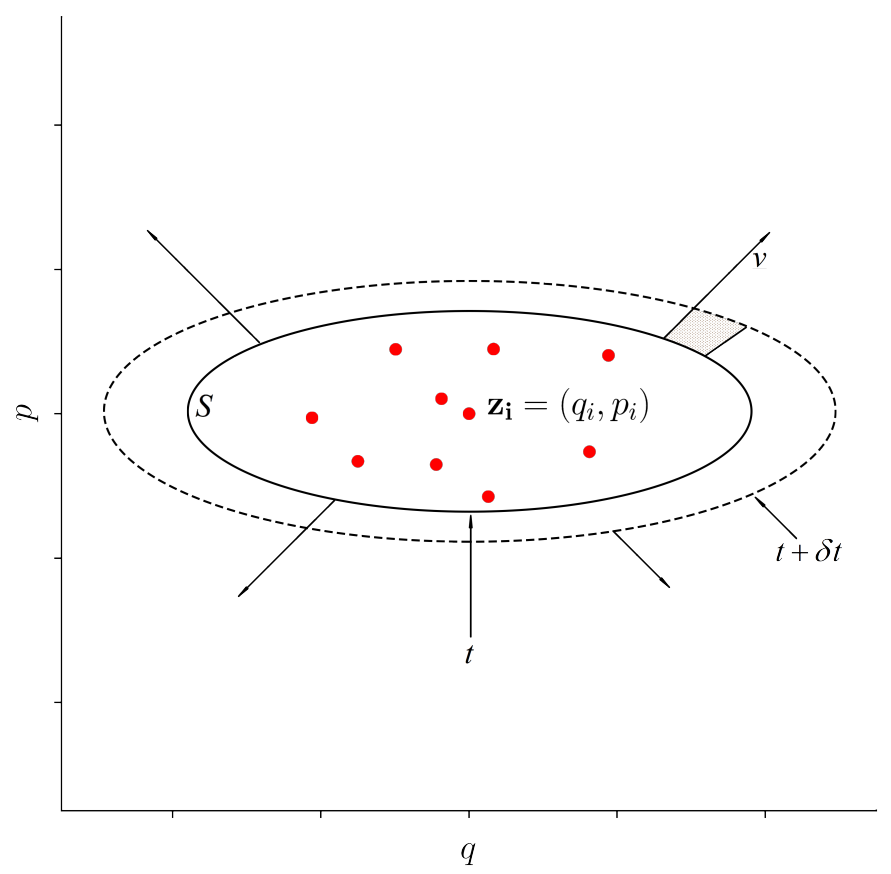

Figura 2 - Curva inicial $S$ do espaço de configuração em linha contínua e região deslocada do espaço de configuração após a passagem de um $\delta t$.

Fonte: Elaborado pelo autor.

espaço de fase 5 a partir do espaço de configurações. Este espaço é construído utilizando a representação das posições $q_{i}$ e dos momentos $p_{j}$ (GREINER, 2003).

Os sistemas Hamiltonianos são ditos serem sistemas conservativos, ou seja, dado um determinado volume $V$ do espaço de fase associado ao sistema dinâmico ocorre a preservação deste volume conforme a evolução do sistema. No caso de sistemas mecânicos, em geral, a quantidade conservada é a energia total associada ao sistema. Este é o principal resultado do teorema de Liouville (TAYLOR, 2005).

Teorema 2.20 (Teorema de Liouville). O fluxo Hamiltoniano autônomo, ou seja, fluxo onde não há dependência explícita do tempo em $\mathscr{H}$, conserva o volume $V$ em seu espaço de configuração.

Para a demonstração do teorema considere o caso especial onde o espaço de fase é bidimensional, considere também uma região $S$ se deslocando do seu estado inicial (curva cheia) com um versor $\mathbf{n}$ normal à curva apontando para fora, figura 2. A velocidade com que os pontos contidos dentro da região $S$ se deslocam é dada por

$$
\mathbf{v}=(\dot{\mathbf{q}}, \dot{\mathbf{p}})=\left(\frac{\partial \mathscr{H}}{\partial p},-\frac{\partial \mathscr{H}}{\partial q}\right) .
$$

5 Por vezes também chamado de espaço de fase Hamiltoniano. 
Para determinar quão rápido o volume $V$ contido em $S$ varia com a trajetória da curva sobre o espaço de fase, consideramos o volume da região infinitesimal pontilhada, vide figura 3, que é dado pela seguinte expressão,

$$
V=\mathbf{v} \cdot \mathbf{n} \delta t \mathrm{~d} A
$$

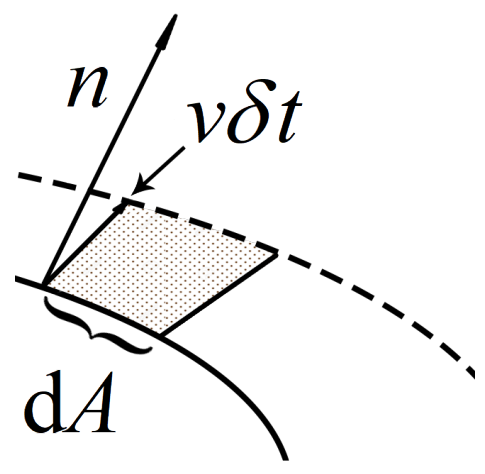

Figura 3 - Elemento infinitesimal de volume no espaço de configurações.

Fonte: Elaborado pelo autor.

O volume de toda a região entre as curvas tracejada e contínua será dada pela soma de cada elemento infinitesimal de volume, o que pode ser expresso em termos de uma integral na seguinte forma,

$$
\delta V=\int_{S} \mathbf{v} \cdot \mathbf{n} \delta t \mathrm{~d} A
$$

Dividindo ambos os lados por $\delta t$ e fazendo $\delta t \rightarrow 0$, temos diretamente

$$
\frac{\mathrm{d} V}{\mathrm{~d} t}=\int_{S} \mathbf{v} \cdot \mathbf{n} \mathrm{d} A
$$

Quando o produto escalar $\mathbf{n} \cdot \mathbf{v}>0$, a região está se expandindo, caso contrário, a região está encolhendo, no entanto, ao longo da curva este produto escalar pode tomar ambos os valores. A equação (18) é válida para qualquer dimensão uma vez que $\mathbf{n} \cdot \mathbf{v}=$ $n_{1} v_{1}+n_{2} v_{2}+\cdots+n_{m} v_{m}$ onde $n_{i}$ e $v_{i}$ são as componentes dos vetores $\mathbf{n}$ e $\mathbf{v}$.

Recorrendo ao teorema da divergência de Gauss,

$$
\int_{S} \mathbf{n} \cdot \mathbf{v} \mathrm{d} A=\int_{V} \nabla \cdot \mathbf{v} \mathrm{d} V
$$

temos que,

$$
\frac{\mathrm{d} V}{\mathrm{~d} t}=\int_{V} \nabla \cdot \mathbf{v} \mathrm{d} V
$$


Observando o divergente temos que,

$$
\nabla \cdot \mathbf{v}=\frac{\partial \dot{q}}{\partial q}+\frac{\partial \dot{p}}{\partial p}=\frac{\partial}{\partial q}\left(\frac{\partial \mathscr{H}}{\partial p}\right)+\frac{\partial}{\partial p}\left(-\frac{\partial \mathscr{H}}{\partial q}\right)=0
$$

$\log 0$

$$
\frac{\mathrm{d} V}{\mathrm{~d} t}=0
$$

Esta conservação do volume do espaço de estado de sistemas Hamiltonianos se deve à ausência de fontes ou sorvedouros na variedade, admitindo apenas pontos de sela ou centros, conservando assim o volume do espaço de fases (VENEGEROLES, 1999).

Dado um Hamiltoniano $\mathscr{H}: \mathbb{R}^{2 n} \rightarrow \mathbb{R}$ de classe $C^{2}$ e seu sistema Hamiltoniano não linear associado $\dot{x}=X_{\mathscr{H}}(x) \operatorname{com} x=(p, q) \in \mathbb{R}^{2 n}$, vale a definição 2.21.

Definição 2.21 (Matriz Hessiana). Uma matriz Hessiana de um Hamiltoniano $\mathscr{H}$ é a matriz $2 n \times 2 n$ com os elementos,

$$
\mathbf{H}_{\left(\mathbf{q}_{0}, \mathbf{p}_{0}\right)}[\mathscr{H}(\mathbf{q}, \mathbf{p})]=\left[\begin{array}{cccc}
\frac{\partial^{2} \mathscr{H}}{\partial q_{1}^{2}} & \frac{\partial^{2} \mathscr{H}}{\partial q_{1} \partial q_{2}} & \cdots & \frac{\partial^{2} \mathscr{H}}{\partial q_{1} \partial p_{n}} \\
\frac{\partial^{2} \mathscr{H}}{\partial q_{2} \partial q_{1}} & \frac{\partial^{2} \mathscr{H}}{\partial q_{2}^{2}} & \cdots & \frac{\partial^{2} \mathscr{H}}{\partial q_{2} \partial p_{n}} \\
\vdots & \vdots & \ddots & \vdots \\
\frac{\partial^{2} \mathscr{H}}{\partial p_{n} \partial q_{1}} & \frac{\partial^{2} \mathscr{H}}{\partial p_{n} \partial q_{2}} & \cdots & \frac{\partial^{2} \mathscr{H}}{\partial p_{n}^{2}}
\end{array}\right]_{\left(\mathbf{q}_{0}, \mathbf{p}_{0}\right)}
$$

Uma matriz de fundamental importância no estudo de sistemas dinâmicos não lineares é a matriz Jacobiana, que é usualmente empregada no processo de linearização de sistemas não lineares.

Definição 2.22 (Matriz Jacobiana). Seja o sistema bidimensional autônomo de equações diferenciais,

$$
\begin{aligned}
& \dot{q}_{1}(t)=f\left(q_{1}, q_{2}\right), \\
& \dot{q}_{2}(t)=g\left(q_{1}, q_{2}\right),
\end{aligned}
$$

a sua matriz Jacobiana associada é definida como

$$
\mathbf{J}\left(q_{1}, q_{2}\right)=\left(\begin{array}{cc}
\frac{\partial f}{\partial q_{1}} & \frac{\partial f}{\partial q_{2}} \\
\frac{\partial g}{\partial q_{1}} & \frac{\partial g}{\partial q_{2}}
\end{array}\right) .
$$

O teorema de Liouville fornece restrições diretas aos autovalores de sistema Hamiltoniano. Estas restrições estão sumarizadas no seguinte teorema 2.23, 
Teorema 2.23. Dado um sistema Hamiltoniano e sua matriz Jacobiana $\mathbf{J}$ associada, existindo um autovalor $\lambda \neq 0$ da matriz $\mathbf{J}$, então $\lambda^{-1}$ também é um autovalor (WRESZINSKI, 1997; VENEGEROLES, 1999).

Como o polinômio característico é de uma variável e usualmente de coeficientes reais, o teorema das raízes complexas assegura a existência autovalores complexos conjugados, assim, dado um autovalor $\lambda \in \mathbb{C}$ de $\mathbf{J}$, seu conjugado complexo também o é, desta forma os autovalores de Hamiltonianos se distribuem em quadruplas,

$$
\lambda, \bar{\lambda}, \lambda^{-1}, \bar{\lambda}^{-1} \quad(|\lambda| \neq 1, \Im(\lambda) \neq 0)
$$

Uma consequência direta desta distribuição de autovalores é a impossibilidade da admissão de pontos fixos assintoticamente estáveis, uma vez que a condição para a existência destes é que existam apenas autovalores com módulo menor do que a unidade. Tomando um autovalor com módulo menor que a unidade $|\lambda|^{2}=a^{2}+b^{2}<1$, é direto observar que $|\lambda|^{-2}>1$, como enunciado pelo teorema 2.23 .

Assim, os pontos fixos instáveis associados a sistemas Hamiltonianos são essencialmente de sela, isto é, não haverão pontos de equilíbrio com ambos os autovalores positivos para formar, por exemplo, um nó instável. A única possibilidade de estabilidade ocorre quando todos os autovalores se encontram na circunferência unitária, caracterizando centros (VENEGEROLES, 1999; WRESZINSKI, 1997).

\subsection{ESTABILIDADE NO SENTIDO DE LYAPUNOV}

Existem diversas noções de estabilidade para um dado sistema dinâmico, no entanto algumas noções são usuais no estudo qualitativo destes sistemas, tais como a de Lyapunov e a Jacobi, que são critérios baseados em diferentes conceitos e métodos (ABOLGHASEM, 2012).

As definições de estabilidade de Lyapunov são dadas nas definições 2.24 e 2.25 como segue de (ABOLGHASEM, 2012).

Definição 2.24 (Estabilidade de Lyapunov). $\overline{\mathbf{x}}(t)$ é dito estável (ou Lyapunov estável) se, dado $\epsilon>0$, existe um $\delta=\delta(\epsilon)>0$ tal que, para qualquer solução $\mathbf{y}(t)$ do sistema (1) satisfazendo $\left|\overline{\mathbf{x}}\left(t_{0}\right)-\mathbf{y}\left(t_{0}\right)\right|<\delta$, então $|\overline{\mathbf{x}}(t)-\mathbf{y}(t)|<\epsilon$ para $t>t_{0}, t_{0} \in \mathbb{R}$. Uma solução da qual não é estável é dita instável. 
Definição 2.25 (Estabilidade assintótica). $\overline{\mathbf{x}}(t)$ é dito assintoticamente estável se é Lyapunov estável e para qualquer outra solução, $\mathbf{y}(t)$ de (1), existe uma constante $b>0$ tal que, se $\left|\overline{\mathbf{x}}\left(t_{0}\right)-\mathbf{y}\left(t_{0}\right)\right|<b$, então $\lim |\mathbf{x}(t)-\mathbf{y}(t)|=0$ quando $t \rightarrow \infty$.

Tendo em mente estas noções de estabilidade, vale o teorema de estabilidade de Lyapunov, enunciado como segue:

Teorema 2.26 (Teorema da estabilidade de Lyapunov). Considere o campo vetorial $\dot{\mathbf{x}}=\mathbf{f}(\mathbf{x}), \mathbf{x} \in \mathbb{R}^{n}$. Seja $\overline{\mathbf{x}}$ um ponto de equilíbrio do campo vetorial $\dot{\mathbf{x}}$ e seja $V: U \rightarrow R$ uma função de classe $C^{1}$, chamada função de Lyapunov, definida em alguma vizinhança $U$ de $\overline{\mathbf{x}}$ tal que,

- $V(\overline{\mathbf{x}})=0$, e $V(\mathbf{x})>0$ se $\mathbf{x} \neq \overline{\mathbf{x}}$,

- $\dot{V}(\mathbf{x}) \leq 0 e m U-\overline{\mathbf{x}}$,

então $\overline{\mathbf{x}}$ é estável. Ainda mais, se

- $\dot{V}(\mathbf{x})<0 e m U-\overline{\mathbf{x}}$

se existir $V$, então $\overline{\mathbf{x}}$ é assintoticamente estável.

Uma outra forma de se analisar qualitativamente um sistema é através de sua estabilidade linear. A título de ilustração, considere o sistema (1) autônomo escrito na forma,

$$
\begin{aligned}
& \dot{u}=f(u, v) \\
& \dot{v}=g(u, v)
\end{aligned},
$$

onde $f(u, v)$ e $g(u, v)$ são funções reais de classe $C^{n}$ com $n \geq 1$. Suponha que exista um ponto fixo em $(0,0)^{6}$ tal que $f(0,0)=g(0,0)=0$. Lineariza-se o sistema via expansão de Taylor para se obter a sua matriz Jacobiana, definida em 2.22. A equação característica do sistema linearizado é obtida igualando o determinante do Jacobiano a zero, assim temos,

$$
\begin{gathered}
\operatorname{det}(\mathbf{J}-\nu \mathbf{I})=\left|\begin{array}{cc}
\frac{\partial f}{\partial u}-\nu & \frac{\partial f}{\partial v} \\
\frac{\partial g}{\partial u} & \frac{\partial g}{\partial v}-\nu
\end{array}\right|=0, \\
\left(\frac{\partial f}{\partial u}-\nu\right)\left(\frac{\partial g}{\partial v}-\nu\right)-\frac{\partial f}{\partial v} \frac{\partial g}{\partial u}=\nu^{2}-\nu\left(\frac{\partial f}{\partial u}+\frac{\partial g}{\partial v}\right)-\frac{\partial f}{\partial v} \frac{\partial g}{\partial u}+\frac{\partial f}{\partial u} \frac{\partial g}{\partial v}=0,
\end{gathered}
$$

6 Sempre é possível fazer uma mudança de variável $\bar{u}=u-u_{0}$ e $\bar{v}=v-v_{0}$ tal que o ponto geral $\left(u_{0}, v_{0}\right)$ vá para a origem do sistema (ABOLGHASEM, 2013). 
de onde é possível perceber que,

$$
\nu^{2}-(\operatorname{tr} A) \nu+\operatorname{det} A=0
$$

onde $\operatorname{tr} A$ é o traço e $\operatorname{det} A$ é o determinante da matriz $\left.A \equiv \mathbf{J}\right|_{(0,0)}$.

A equação característica é polinomial de grau dois, assim suas raízes são dadas via

$$
\nu_{1,2}=\frac{\operatorname{tr} A \pm \sqrt{\Delta}}{2}
$$

onde podemos definir o discriminante

$$
\Delta \equiv(\operatorname{tr} A)^{2}-4 \operatorname{det} A
$$

Observa-se diretamente da equação (32) que a curva onde $\Delta=0$ é uma parábola positiva. A estabilidade linear pode ser sintetizada no esquema gráfico apresentado na figura 4, onde é realizada a comparação com a estabilidade de Jacobi.

\subsection{ESTABILIDADE NO SENTIDO DE JACOBI}

A investigação a respeito da estabilidade dos sistemas dinâmicos recai sobre diferentes métodos e noções de estabilidade, sendo uma das mais importantes a noção de Lyapunov, discutida na seção 2.7. Uma noção alternativa se dá pela estabilidade de Jacobi. A análise de estabilidade via Jacobi utiliza-se de elementos da teoria KCC (Kosambi-Cartan-Chern) para caracterizar os desvios de trajetórias vizinhas no retrato de fases. As definições de apresentadas aqui seguem do trabalho de Sabau (2005) e Boehmer et al. (2012). Inicialmente, consideremos o conjunto de variáveis dinâmicas do sistema

$$
\left(x^{1}, x^{2}, \ldots, x^{n}\right)=(x)
$$

as taxas de variação com relação à variável independente $\lambda$

$$
\left(\frac{\mathrm{d} x^{1}}{\mathrm{~d} \lambda}, \frac{\mathrm{d} x^{2}}{\mathrm{~d} \lambda}, \frac{\mathrm{d} x^{3}}{\mathrm{~d} \lambda}, \ldots, \frac{\mathrm{d} x^{n}}{\mathrm{~d} \lambda}\right)=\left(\frac{\mathrm{d} x}{\mathrm{~d} \lambda}\right)=\dot{x}
$$

e a própria variável independente $\lambda$ como as $2 n+1$ coordenadas de um subconjunto $\Omega$ aberto e conectado do espaço euclidiano $\mathbb{R}^{n} \times \mathbb{R}^{n} \times \mathbb{R}^{1}$. Consideremos também a equação diferencial de segunda ordem generalizada,

$$
\frac{\mathrm{d}^{2} x^{i}}{\mathrm{~d} \lambda^{2}}+G^{i}(x, \dot{x}, \lambda)=0, \quad i \in\{1,2, \ldots, n\}
$$


onde $G^{i}(\lambda, x, \dot{x})$ é uma função de classe $C^{\infty}$ em uma vizinhança de alguma condição inicial $\left((x)_{0},(\dot{x})_{0}, \lambda_{0}\right)$ de $\Omega$.

A princípio, para que possamos encontrar alguns invariantes diferenciais em relação a uma transformação não singular do tipo,

$$
\tilde{x}^{i}=f^{i}\left(x^{1}, x^{2}, \ldots, x^{n}\right) \quad \text { com } \quad i \in\{1,2, \ldots, n\}, \quad \bar{\lambda}=\lambda
$$

definimos a KCC-diferencial covariante de um campo vetorial contravariante $\xi^{i}(x)$ como segue:

Definição 2.27 (KCC-diferencial covariante). Seja um campo vetorial contravariante $\xi^{i}(x)$ definido sobre $\Omega$, define-se o diferencial covariante da seguinte forma,

$$
\frac{\mathrm{D} \xi^{i}}{\mathrm{~d} \lambda}=\frac{\mathrm{d} \xi^{i}}{\mathrm{~d} \lambda}+\frac{1}{2} \frac{\partial G^{i}}{\partial \dot{x}^{r}} \xi^{r}
$$

onde há a convenção da soma implícita (convenção da soma de Einstein), isto é, soma-se sobre os índices repetidos no mesmo termo.

Derivando com relação à $\lambda$ a equação (37) aplicada a $x^{i}(\lambda)$, temos,

$$
\frac{\mathrm{D} \dot{x}^{i}}{\mathrm{~d} \lambda}=\frac{\mathrm{d}^{2} x^{i}}{\mathrm{~d} \lambda^{2}}+\frac{1}{2} \frac{\partial G^{i}}{\partial \dot{x}^{r}} \dot{x}^{r}
$$

que, com o auxílio da equação (35) pode ser expressa da seguinte forma,

$$
\frac{\mathrm{D} \dot{x}^{i}}{\mathrm{~d} \lambda}=\frac{1}{2} \frac{\partial G^{i}}{\partial \dot{x}^{r}} \dot{x}^{r}-G^{i} \equiv \epsilon^{i}
$$

onde o campo vetorial contravariante $\epsilon^{i}$ é o chamado primeiro $K C C$-invariante, usualmente interpretado como as forças externas do sistema.

Variando as trajetórias $x^{i}(\lambda)$ e em trajetórias infinitesimalmente próximas, dadas por

$$
\tilde{x}^{i}=x^{i}-\eta \xi^{i}(\lambda)
$$

onde $|\eta| \ll 1$ e $\xi^{i}(\lambda)$ são as componentes de algum campo contravariante definido ao longo da solução $x^{i}(\lambda)$. Como $\tilde{x}$ e $x$ são soluções de (35), derivando-se duas vezes a expressão (40), vemos que,

$$
\eta \ddot{\xi}^{i}(\lambda)+(\tilde{G}-G)=0
$$

onde

$$
\tilde{G}^{i}-G^{i} \equiv G^{i}(\lambda, x+\eta \xi, \dot{x}+\eta \dot{\xi})-G^{i}(\lambda, x, \dot{x})
$$


Encarando $\tilde{G}-G$ como uma função do parâmetro $\eta$ e aplicando o teorema do valor médio, observa-se que

$$
\tilde{G}^{i}(\lambda, x+\eta \xi, \dot{x}+\eta \dot{\xi})-G^{i}(\lambda, x, \dot{x})=\eta \frac{\mathrm{d} G}{\mathrm{~d} \eta},
$$

desta forma, a nossa equação variacional assume a seguinte forma,

$$
\ddot{\xi}^{i}+\frac{\mathrm{d} G^{i}}{\mathrm{~d} \eta}=0
$$

Da equação (42), diferenciamos utilizando a regra da cadeia nas expressões para obter

$$
\ddot{\xi}^{i}+\frac{\partial G^{i}}{\partial x^{r}} \frac{\mathrm{d} x^{r}}{\mathrm{~d} \eta}+\frac{\partial G^{i}}{\partial \dot{x}^{r}} \frac{\mathrm{d} \dot{x}^{r}}{\mathrm{~d} \eta} .
$$

Derivando a expressão (40) em relação à $\eta$, temos

$$
\frac{\mathrm{d} x^{i}}{\mathrm{~d} \eta}=\xi^{i}
$$

Agora, derivando a expressão (40) com relação à $\lambda$ e derivando novamente com relação à $\eta$, temos

$$
\frac{\mathrm{d} \dot{x}^{i}}{\mathrm{~d} \eta}=\frac{\mathrm{d} \xi^{i}}{\mathrm{~d} \lambda} .
$$

Colecionando os resultados (46) e (47), e substituindo na expressão (45), temos a seguinte equação

$$
\ddot{\xi}^{i}+\frac{\partial G^{i}}{\partial x^{r}} \xi^{r}+\frac{\partial G^{i}}{\partial \dot{x}^{r}} \dot{\xi}^{r}=0 .
$$

Para colocar a equação variacional na sua forma contravariante, utilizamos a definição 2.27 para obtermos

$$
\frac{\mathrm{D}^{2} \xi^{i}}{\mathrm{~d} \lambda^{2}}=\frac{\mathrm{D}}{\mathrm{d} \lambda} \frac{\mathrm{D} \xi^{i}}{\mathrm{~d} \lambda}=\frac{\mathrm{D}}{\mathrm{d} \lambda}\left(\frac{\mathrm{d} \xi^{i}}{\mathrm{~d} \lambda}+\frac{1}{2} \frac{\partial G^{i}}{\partial \dot{x}^{r}} \xi^{r}\right),
$$

de onde vemos que aplicando o operador diferencial chega-se à seguinte equação,

$$
\frac{\mathrm{D}^{2} \xi^{i}}{\mathrm{~d} \lambda^{2}}=\frac{\mathrm{d}}{\mathrm{d} \lambda}\left(\frac{\mathrm{d} \xi^{i}}{\mathrm{~d} \lambda}+\frac{1}{2} \frac{\partial G^{i}}{\partial \dot{x}^{r}} \xi^{r}\right)+\frac{1}{2} \frac{\partial G^{i}}{\partial \dot{x}^{j}}\left(\frac{\mathrm{d} \xi^{j}}{\mathrm{~d} \lambda}+\frac{1}{2} \frac{\partial G^{j}}{\partial \dot{x}^{r}} \xi^{r}\right),
$$

que após abertos os termos da soma fornece,

$$
\frac{\mathrm{D}^{2} \xi^{i}}{\mathrm{~d} \lambda^{2}}=\frac{\mathrm{d}^{2} \xi^{i}}{\mathrm{~d} \lambda^{2}}+\frac{1}{2} \frac{\partial G^{i}}{\partial \dot{x}^{r}} \frac{\mathrm{d} \xi^{r}}{\mathrm{~d} \lambda}+\frac{1}{2}\left(\frac{\mathrm{d}}{\mathrm{d} \lambda} \frac{\partial G^{i}}{\partial \dot{x}^{r}}\right) \xi^{r}+\frac{1}{2} \frac{\partial G^{i}}{\partial \dot{x}^{j}} \frac{\mathrm{d} \xi^{j}}{\mathrm{~d} \lambda}+\frac{1}{4} \frac{\partial G^{i}}{\partial \dot{x}^{j}} \frac{\partial G^{j}}{\partial \dot{x}^{r}} \xi^{r} .
$$

Pelo fato de que existem dois índices mudos nas expressões, a saber $r$ e $j$, podemos construir a seguinte igualdade,

$$
\frac{1}{2} \frac{\partial G^{i}}{\partial \dot{x}^{r}} \frac{\mathrm{d} \xi^{r}}{\mathrm{~d} \lambda}=\frac{1}{2} \frac{\partial G^{i}}{\partial \dot{x}^{j}} \frac{\mathrm{d} \xi^{j}}{\mathrm{~d} \lambda}
$$


que simplifica a equação (51),

$$
\frac{\mathrm{D}^{2} \xi^{i}}{\mathrm{~d} \lambda^{2}}=\frac{\mathrm{d}^{2} \xi^{i}}{\mathrm{~d} \lambda^{2}}+\frac{\partial G^{i}}{\partial \dot{x}^{r}} \frac{\mathrm{d} \xi^{r}}{\mathrm{~d} \lambda}+\frac{1}{2}\left(\frac{\mathrm{d}}{\mathrm{d} \lambda} \frac{\partial G^{i}}{\partial \dot{x}^{r}}\right) \xi^{r}+\frac{1}{4} \frac{\partial G^{i}}{\partial \dot{x}^{j}} \frac{\partial G^{j}}{\partial \dot{x}^{r}} \xi^{r}
$$

Do resultado (48) vemos que

$$
\frac{\mathrm{d}^{2} \xi^{i}}{\mathrm{~d} \lambda^{2}}=-\frac{\partial G^{i}}{\partial \dot{x}^{r}} \frac{\mathrm{d} \xi^{r}}{\mathrm{~d} \lambda}-\frac{\partial G^{i}}{\partial x^{r}} \xi^{r}
$$

desta forma, substituímos a expressão (54) na equação (53),

$$
\frac{\mathrm{D}^{2} \xi^{i}}{\mathrm{~d} \lambda^{2}}=-\frac{\partial G^{i}}{\partial x^{r}} \xi^{r}+\frac{1}{2}\left(\frac{\mathrm{d}}{\mathrm{d} \lambda} \frac{\partial G^{i}}{\partial \dot{x}^{r}}\right) \xi^{r}+\frac{1}{4} \frac{\partial G^{i}}{\partial \dot{x}^{j}} \frac{\partial G^{i}}{\partial \dot{x}^{r}} \xi^{r}
$$

Para expandir o termo em parênteses devemos observar que uma função $F$ parametrizada por $\lambda$

$$
F(\lambda, x(\lambda), \dot{x}(\lambda)) \equiv \partial_{\dot{x}^{r}} G^{i}
$$

possui diferenciação total com relação à $\lambda$ dada por

$$
\frac{\mathrm{d} F}{\mathrm{~d} \lambda}=\frac{\partial F}{\partial \lambda}+\frac{\partial F}{\partial x^{j}} \frac{\partial x^{j}}{\partial \lambda}+\frac{\partial F}{\partial \dot{x}^{j}} \frac{\partial \dot{x}^{j}}{\partial \lambda}
$$

que com o auxílio da expressão (35), nos fornece,

$$
\frac{\mathrm{d} F}{\mathrm{~d} \lambda}=\frac{\partial F}{\partial \lambda}+\frac{\partial F}{\partial x^{j}} \frac{\partial x^{j}}{\partial \lambda}-G^{j} \frac{\partial F}{\partial \dot{x}^{j}} .
$$

Finalmente, unindo as equações (58) e (55) para obter,

$$
\frac{\mathrm{D}^{2} \xi^{i}}{\mathrm{~d} \lambda^{2}}=-\frac{\partial G^{i}}{\partial x^{r}} \xi^{r}+\frac{1}{2}\left(\frac{\partial}{\partial \lambda} \frac{\partial G^{i}}{\partial \dot{x}^{r}}+\frac{\partial}{\partial x^{j}} \frac{\partial G^{i}}{\partial \dot{x}^{r}} \frac{\partial x^{j}}{\partial \lambda}-G^{j} \frac{\partial}{\partial \dot{x}^{j}} \frac{\partial G^{i}}{\partial \dot{x}^{r}}\right) \xi^{r}+\frac{1}{4} \frac{\partial G^{i}}{\partial \dot{x}^{j}} \frac{\partial G^{j}}{\partial \dot{x}^{r}} \xi^{r} .
$$

A equação (59) pode ser rearranjada para nos fornecer a seguinte expressão,

$$
\frac{\mathrm{D}^{2} \xi^{i}}{\mathrm{~d} \lambda^{2}}=P_{r}^{i} \xi^{r}
$$

onde

$$
P_{r}^{i} \equiv-\frac{\partial G^{i}}{\partial x^{r}}+\frac{1}{2}\left(\frac{\partial}{\partial \lambda} \frac{\partial G^{i}}{\partial \dot{x}^{r}}+\frac{\partial}{\partial x^{j}} \frac{\partial G^{i}}{\partial \dot{x}^{r}} \dot{x}^{j}-G^{j} \frac{\partial}{\partial \dot{x}^{j}} \frac{\partial G^{i}}{\partial \dot{x}^{r}}\right)+\frac{1}{4} \frac{\partial G^{i}}{\partial \dot{x}^{j}} \frac{\partial G^{j}}{\partial \dot{x}^{r}}
$$

é o chamado segundo KCC-invariante ou tensor de curvatura de desvio.

Definindo a conexão não-linear $N_{r}^{i}$ e a conexão de Berwald $G_{j l}^{i}$ respectivamente como

$$
N_{r}^{i} \equiv \partial_{\dot{x}^{r}} G^{i}, \quad G_{j l}^{i} \equiv \partial_{\dot{x}^{l}} N_{j}^{i}
$$


podemos expressar este tensor como (BOEHMER et al., 2012; ABOLGHASEM, 2013; ABOLGHASEM, 2012),

$$
P_{r}^{i}=-\frac{\partial G^{i}}{\partial x^{r}}-\frac{1}{2} G^{j} G_{r j}^{i}+\frac{1}{2} \frac{\partial N_{r}^{i}}{\partial x^{j}} \dot{x}^{j}+\frac{1}{4} N_{j}^{i} N_{r}^{j}+\frac{1}{2} \frac{\partial N_{r}^{i}}{\partial \lambda} .
$$

A análise de estabilidade via Jacobi é dada pelo sinal das partes reais dos autovalores associados ao tensor (63), e pode ser definida conforme apresentado a seguir.

Definição 2.28 (Estabilidade de Jacobi). As trajetórias de (35) são estáveis no sentido de Jacobi se, e somente se, a parte real dos autovalores de $P_{r}^{i}$ são estritamente negativas, caso contrário, as trajetórias são ditas instáveis no sentido de Jacobi.

A instabilidade no sentido de Jacobi está relacionada com o crescimento exponencial do campo vetorial $\xi$ enquanto a estabilidade está associada a uma variação oscilatória do campo $\xi$. As condições de estabilidade são, em termos do traço, determinante e discriminante.

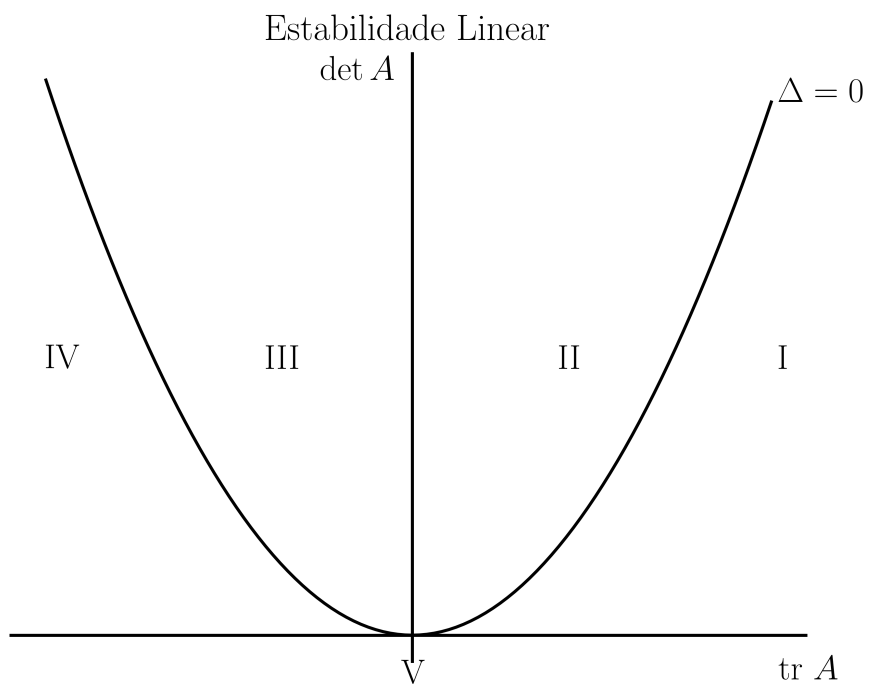

Figura 4 - Regiões da análise de estabilidade linear.

Fonte: Elaborado pelo autor.

\begin{tabular}{|c|c|c|c|c|c|}
\hline Região & Lyapunov & Jacobi & $\operatorname{det}(A)$ & $\operatorname{Tr}(A)$ & $\Delta$ \\
\hline \hline I & Instável & Instável & $>0$ & $>0$ & $>0$ \\
II & Instável & Estável & $>0$ & $>0$ & $<0$ \\
III & Estável & Estável & $>0$ & $<0$ & $<0$ \\
IV & Estável & Instável & $>0$ & $<0$ & $>0$ \\
V & Instável & Instável & $<0$ & $=0$ & $>0$ \\
\hline
\end{tabular}

Quadro 2 - Quadro de comparação entre a estabilidade de Lyapunov e de Jacobi. 
Pode-se perceber que a estabilidade linear não está de acordo com a estabilidade de Jacobi em todas as situações. Isto ocorre pois a estabilidade no sentido de Jacobi se refere a uma estabilidade de trajetórias em um espaço curvo dotado de uma conexão não linear e um tensor de curvatura. Nesta noção de estabilidade a diferenciação covariante desempenha o papel da derivação parcial usual, gerando assim as diferenças encontradas. Para uma discussão detalhada sobre a relação entre a estabilidade linear e a estabilidade via Jacobi veja, por exemplo, a revisão apresentada por Sabau (2005) ou os trabalhos de aplicação feitos por Abolghasem (2013) e Boehmer et al. (2012).

\subsection{BIFURCAÇÕES NO ESPAÇO DE ESTADOS}

Um sistema dinâmico usualmente depende de parâmetros, que ao serem variados podem gerar mudanças topológicas relevantes no espaço de estados do sistema. Quando existe tal mudança na topologia do espaço de estados do sistema em decorrência da variação dos seus parâmetros, usualmente é dito que o sistema passou por uma bifurcação. Para uma definição mais formal, é necessário introduzir a noção de equivalência topológica.

Definição 2.29 (Equivalência Topológica). Sejam dois sistemas dinâmicos $D_{1}=\left\{T, \mathbb{R}^{n}, \phi^{t}\right\}$ e $D_{2}=\left\{T, \mathbb{R}^{n}, \psi^{t}\right\}$. É dito que $D_{1}$ é topologicamente equivalente à $D_{2}$ se existe um homeomorfismo $h: \mathbb{R}^{n} \mapsto \mathbb{R}^{n}$ mapeando as órbitas do primeiro sistema no segundo, preservando a direção do tempo (KUZNETSOV, 2013).

A existência de um homeomorfismo $h$ implica que há um mapeamento contínuo e de inversa contínua entre os sistemas, assim, podemos escrever de forma esquemática (KUZNETSOV, 2013)

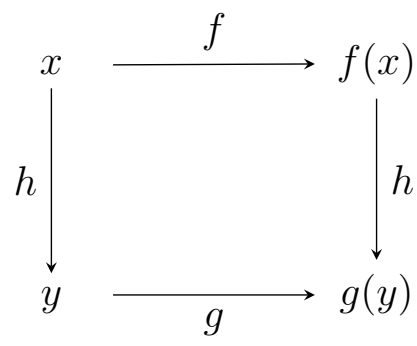

Figura 5 - Esquema simbólico de um homeomorfismo $h: \mathbb{R}^{n} \mapsto \mathbb{R}^{n}$.

Fonte: Elaborado pelo autor. 
De uma forma mais compacta, temos que

$$
f(x)=h^{-1}(g(h(x))),
$$

com $x \in \mathbb{R}^{n}, y \in \mathbb{R}^{n}$ e $h$ uma função contínua invertível entre $\mathbb{R}^{n}$ e $\mathbb{R}^{n}$. A existência de um homeomorfismo assegura que ambos os espaços de estados terão as mesmas estruturas topológicas, tais como pontos fixos ou ciclo limites, assegurando também que a orientação das soluções é mantida. Neste cenário, bifurcações ocorrem quando é quebrada a equivalência topológica entre os espaços de estados do sistema com parâmetros perturbados e não perturbados.

Definição 2.30 (Bifurcação). O surgimento de um retrato de fases topologicamente não equivalente sobre variação dos parâmetros do sistema é chamado de bifurcação (KUZNETSOV, 2013).

A variação da quantidade de pontos fixos do sistema, bem como trocas de estabilidade destes pontos fixos são exemplos de mecanismos geradores de bifurcações em sistemas dinâmicos. Um primeiro exemplo de bifurcação é a chamada bifurcação fold, definida em 2.31 .

Definição 2.31 (Bifurcação fold). Seja o sistema dinâmico (1) dependendo apenas de um parâmetro. A bifurcação associada ao aparecimento de um autovalor $\nu_{1}=0$ da matriz Jacobiana $\mathbf{J}$ avaliada no ponto fixo $x_{0} \in \mathbb{R}$ é chamada de bifurcação fold, sela-nó ou blue-sky (KUZNETSOV, 2013).

Eventualmente, é possível que os autovalores cruzem o eixo imaginário, gerando um par de autovalores complexos e conjugados, puramente imaginários, esta bifurcação é denominada Andronov-Hopf, como na definição 2.32.

Definição 2.32 (Bifurcação Andronov-Hopf). Seja o sistema dinâmico (1) com $x \in \mathbb{R}^{n}$ com $n \geq 2$ e $\nu_{1,2}$ os autovalores da matriz $\mathbf{J}$ avaliada no ponto fixo $x_{0} \in \mathbb{R}^{2}$. A bifurcação correspondendo à presença de $\nu_{1,2}= \pm i \omega_{0}$ com $\omega_{0}>0$ é chamada de bifurcação de Andronov-Hopf (KUZNETSOV, 2013).

Uma bifurcação muito comum em sistemas conservativos (logo, também nos sistemas Hamiltonianos) é a chamada bifurcação centro-sela, definida em 2.33. 
Definição 2.33 (Bifurcação centro-sela). Seja o sistema dinâmico (1) dependendo apenas de um parâmetro. A bifurcação associada à coalescência de um ponto fixo do tipo sela com um tipo centro, aniquiliando ambos após a bifurcação, é chamada de bifurcação centro-sela (HALE; KOÇAK, 2012).

No âmbito das bifurcações dos sistemas dinâmicos que dependem de dois parâmetros ou mais, temos a chamada bifurcação Bogdanov-Takens (BBT), definida em 2.34.

Definição 2.34 (Bifurcação de Bogdanov-Takens). Seja o sistema dinâmico autônomo (1) com $x \in \mathbb{R}^{n}$ e $\mu \in \mathbb{R}^{m}$ onde $m, n \geq 2$. Seja $\nu_{1,2}=0$ os autovalores da matriz $\mathbf{J}$ associados algum ponto fixo da dinâmica. Se o bloco de Jordan

$$
\mathbf{J}\left(x_{0}\right)=\left(\begin{array}{ll}
0 & 1 \\
0 & 0
\end{array}\right),
$$

está associado a estes autovalores, então possui uma bifurcação de Bogdanov-Takens (KUZNETSOV, 2005; KUZNETSOV, 2013).

O diagrama completo de bifurcação de uma Bogdanov-Takens envolve bifurcações do tipo homoclínica, Andronov-Hopf, centro-sela, dentre outras, como mostrado na figura 6 , onde $\beta_{1}, \beta_{2}$ são os parâmetros da forma normal (69). O ramo vertical $H$ é um ramo onde ocorre uma bifurcação Andronov-Hopf. Os ramos $T_{+}$e $T_{-}$são os ramos de uma bifurcação fold, enquanto o ramo $P$ é um ramo de bifurcação homoclínica.

O estudo de bifurcações recai sobre o estudo de formais normais, que são os sistemas dinâmicos mais simples que apresentam o comportamento da bifurcação em estudo. Por exemplo, a bifurcação fold exibe a seguinte forma normal,

$$
\dot{x}=x^{2}+\mu \equiv f(x) \text {. }
$$

Observa-se que a função $f(x)$ é uma parábola de curvatura positiva, cujas raízes são

$$
x_{1,2}= \pm i \sqrt{\mu} .
$$

Assim, só existem pontos fixos para $\mu \leq 0$. Quando $\mu<0$ temos dois pontos fixos distintos e com estabilidades opostas, enquanto para $\mu=0$, há apenas um ponto fixo do 


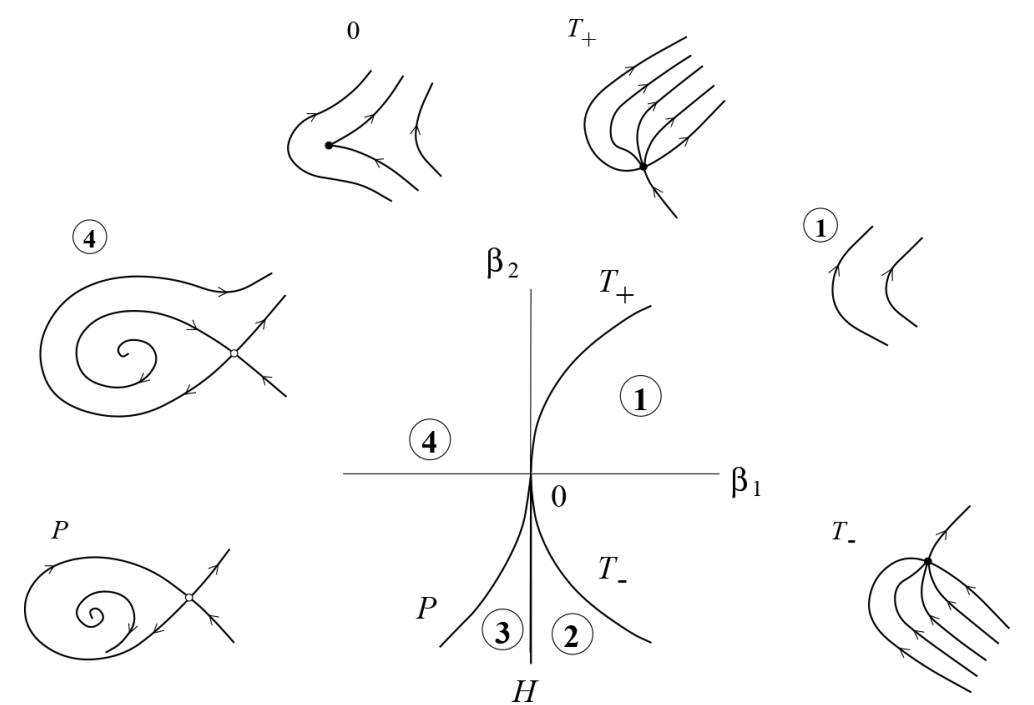

(3)

(2),H
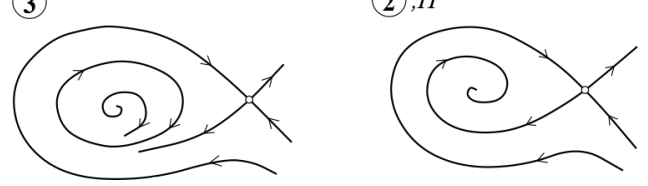

Figura 6 - Diagrama completo da bifurcação Bogdanov-Takens.

Fonte: Kuznetsov (2013).

tipo sela. A forma normal de um bifurcação de Bogdanov-Takens não degenerada, isto é $a_{2} b_{2} \neq 0$, é dada pelo seguinte sistema (KUZNETSOV, 2013),

$$
\begin{aligned}
& \dot{x_{1}}=x_{2}, \\
& \dot{x_{2}}=\beta_{1}+\beta_{2} x_{1}+a_{2} x_{1}^{2}+b_{2} x_{1} x_{2} .
\end{aligned}
$$

Todavia é possível que ocorra $b_{2}=0$, neste caso, se $a_{2} \neq 0$ o sistema (69) pode ser transformado por uma mudança suave de coordenadas e reparametrização temporal no seguinte sistema (KUZNETSOV, 2005),

$$
\begin{aligned}
& \dot{x_{1}}=x_{2}, \\
& \dot{x_{2}}=a_{2} x_{1}^{2}+b_{4} x_{1}^{3} x_{2}+\mathcal{O}\left(\left\|x_{1}, x_{2}\right\|^{5}\right) .
\end{aligned}
$$

Este tipo de bifurcação foi extensivamente estudado por Rifkat Bogdanov (BOGDANOV, 1975) e por Floris Takens (TAKENS, 1973), onde a descrição inicial foi realizada, posteriormente um método prático para o cálculo dos coeficientes das formas normais desta bifurcação foi proposto por Yuri Kuznetsov (KUZNETSOV, 2005), e será o método utilizado neste trabalho. 


\section{GRAVITAÇÃO E RELATIVIDADE GERAL}

Einstein em 1916 consolidou em seu artigo Grundlage der allgemeinen Relativitätstheorie (Fundações da Teoria da Relatividade) uma série de artigos onde era discutida e proposta uma interpretação geométrica da gravidade. Nesta nova abordagem, diversos elementos de geometria diferencial foram utilizados para a apresentação de uma interpretação geométrica da gravitação, onde uma entidade chamada espaço-tempo era o principal agente dos fenômenos gravitacionais. A definição formal de espaço-tempo no contexto da relatividade geral é dada pela definição 3.1 .

Definição 3.1 (Espaço-tempo). O espaço-tempo é uma variedade quadridimensional suave, conexa e Lorentziana (MISNER; THORNE; WHEELER, 2017).

Definição 3.2 (Variedade conexa). Uma variedade $X$ é dita conexa quando as seguintes condições são satisfeitas: sejam $A, B \subset X$ dois conjuntos abertos tais que não há uma outra cisão que não seja a trivial, ou seja, se $X=A \cup B \operatorname{com} A \cap B=\emptyset, \log$ $A=\emptyset$ ou $B=\emptyset($ MUNKRES, 2000).

Em relatividade geral, as quantidades associadas ao espaço-tempo são avaliadas com o auxílio da métrica, definida como segue.

Definição 3.3 (Métrica). A métrica, no contexto da relatividade geral, é uma forma bilinear, simétrica e não degenerada (O’NEILL, 1983).

Em termos de uma base covariante a métrica é expressa por uma matriz $g_{\mu \nu}$. A métrica é usualmente apresentada de forma alternativa pelo chamado elemento de linha. O elemento de linha é o traço da matriz $g_{\mu \nu}$

$$
\mathrm{d} s^{2}=g_{\mu \nu} \mathrm{d} q^{\mu} \mathrm{d} q^{\nu}
$$

onde existe, neste caso, a soma implícita que deve ser tomada sobre índices covariantes e contravariantes que se repetem (notação de Einsten).

Definição 3.4 (Métrica Lorentziana). Uma variedade é dita Lorentziana caso a sua métrica possua assinatura $(-,+,+,+)$ ou $(+,-,-,-)$ (O’NEILL, 1983).

Neste capítulo serão introduzidas algumas quantidades usuais no estudo da relatividade geral e deduzidas as equações de movimento geodésico a partir do formalismo Hamiltoniano. 


\subsection{QUANTIDADES DE CURVATURA}

A relatividade geral lança uso de geometria diferencial para explicar os efeitos gravitacionais, traçando um caráter geométrico às suas interpretações. Para realizar uma descrição de como a matéria perturba o espaço-tempo é necessário estudar espaços curvos, tarefa que é realizada com o auxílio da geometria Riemanniana (WEINBERG; DICKE, 1973).

Definição 3.5 (Vetores contravariantes). Um vetor contravariante é um vetor que está contido no espaço tangente à variedade em um ponto $p$, ou seja, em termos de uma base $e_{\mu}$ estes vetores podem ser expressos como

$$
V=V^{\mu} \mathrm{e}_{\mu}
$$

Definição 3.6 (Vetores covariantes). Um vetor covariante é um vetor que está contido no espaço cotangente à variedade em um ponto $p$, ou seja, em termos de uma base $e^{\mu}$ estes vetores podem ser expressos como

$$
V=V_{\mu} \mathrm{e}^{\mu}
$$

A relação entre vetores covariantes e contravariantes pode ser realizada utilizando a métrica, por exemplo,

$$
\begin{aligned}
& U^{\nu}=g^{\mu \nu} U_{\mu}, \\
& U_{\mu}=g_{\mu \nu} U^{\nu},
\end{aligned}
$$

sendo que o tensor métrico $g_{\mu \nu}$ se relaciona com as formas covariante e contravariante segundo a relação

$$
g^{\mu \lambda} g_{\lambda \nu}=\delta_{\nu}^{\mu}
$$

A quantidade $\delta_{\nu}^{\mu}$ é apresentada na definição 3.7.

Definição 3.7 (Delta de Kronecker). A matriz $\delta_{\nu}^{\mu}$ é definida como

$$
\delta_{\nu}^{\mu}=\left\{\begin{array}{lll}
1 & \text { se } & \mu=\nu, \\
0 & \text { se } & \mu \neq \nu .
\end{array}\right.
$$

Em relatividade a conexão é escolhida de forma a ser compatível com a métrica, como veremos a seguir. Os símbolos de Christoffel são definidos como segue em 3.8. 
Definição 3.8 (Símbolos de Christoffel). No contexto da relatividade geral, a conexão é dada pela quantidade chamada símbolo de Christoffel,

$$
\Gamma_{\mu \nu}^{\lambda}=\frac{1}{2} g^{\lambda \sigma}\left(\partial_{\mu} g_{\nu \sigma}+\partial_{\nu} g_{\sigma \mu}-\partial_{\sigma} g_{\mu \nu}\right)
$$

Esta quantidade não é um tensor, pois não segue a lei de transformação de tensores (CARROLL, 2004).

Os símbolos de Christoffel funcionam como uma correção da diferenciação em espaços curvos, estando assim presentes na definição de derivada covariante de um tensor $T^{\mu \nu}$ qualquer.

Definição 3.9 (Derivada covariante). A derivada covariante de um tensor $T^{\mu \nu}$ é dada pela seguinte expressão,

$$
\nabla_{\mu} T^{\mu \nu}=\partial_{\mu} T^{\mu \nu}+\Gamma_{\mu \lambda}^{\mu} T^{\lambda \nu}+\Gamma_{\mu \lambda}^{\nu} T^{\mu \lambda}
$$

O fato da conexão ser compatível com a métrica significa que a derivada covariante do tensor métrico é zero, ou seja $\nabla_{\mu} g^{\mu \nu}=0$, e que a torsão é nula (MISNER; THORNE; WHEELER, 2017). Uma medida de quão curvo é o espaço em questão é o chamado tensor de curvatura ou tensor de Riemann-Christoffel.

Definição 3.10 (Tensor de Riemann-Christoffel). O tensor de Riemann-Christoffel é definido sobre uma variedade como a quantidade

$$
R_{\sigma \mu \nu}^{\lambda}=\partial_{\nu} \Gamma_{\mu \nu}^{\lambda}-\partial_{\mu} \Gamma_{\nu \sigma}^{\lambda}+\Gamma_{\alpha \nu}^{\lambda} \Gamma_{\mu \sigma}^{\alpha}-\Gamma_{\alpha \mu}^{\lambda} \Gamma_{\sigma \nu}^{\alpha}
$$

Ao se realizar uma contração de índices no tensor de curvatura (81), obtemos o tensor de Ricci.

Definição 3.11 (Tensor de Ricci). Seja $R_{\sigma \mu \nu}^{\lambda}$ o tensor de curvatura de Riemann-Christoffel. A contração de dois índices deste tensor origina o tensor de Ricci $\left(R_{\lambda \mu}\right)$

$$
R_{\lambda \mu}=R_{\mu \lambda}=\partial_{\mu} \Gamma_{\lambda \sigma}^{\sigma}-\partial_{\sigma} \Gamma_{\lambda \mu}^{\sigma}+\Gamma_{\lambda \sigma}^{\tau} \Gamma_{\mu \tau}^{\sigma}-\Gamma_{\lambda \mu}^{\tau} \Gamma_{\tau \sigma}^{\sigma} \equiv R_{\lambda \mu \sigma}^{\sigma}
$$

Realizando uma outra contração sobre um dos índices do tensor de Ricci, temos o chamado escalar de curvatura, ou equivalentemente escalar de Ricci ou ainda invariante de curvatura (CARROLL, 2004). 
Definição 3.12 (Escalar de Ricci). A contração dos índices do tensor de curvatura de Ricci fornece o escalar de Ricci $(R)$,

$$
R \equiv g^{\lambda \sigma} R_{\sigma \lambda}
$$

que também é uma medida associada à curvatura do espaço (CATTANI, 1998).

\subsection{AS EQUAÇÕES DE CAMPO DE EINSTEIN}

A equação que modela a dinâmica gravitacional proposta por Einstein é a seguinte (EINSTEIN, 1916),

$$
G_{\mu \nu}+\Lambda g_{\mu \nu}=\kappa T_{\mu \nu}
$$

onde $G_{\mu \nu}$ é o tensor de Einstein, dado por

$$
G_{\mu \nu} \equiv R_{\mu \nu}-\frac{1}{2} R g_{\mu \nu}
$$

com

$$
\kappa=\frac{8 \pi G}{c^{4}}
$$

Nas equações de Einstein $\Lambda$ é a constante cosmológica, $G$ é a constante gravitacional universal e $c$ é a velocidade da luz no vácuo. É possível reescalar o sistema de unidades de forma que as constantes assumam o valor $c=G=1$. Este será o sistema de unidades utilizado daqui em diante, a menos que seja previamente informado o contrário.

O tensor de Einstein está relacionado com a deformação do espaço-tempo que é causada pela distribuição de matéria e energia, enquanto o lado direito da equação (84) está associado à distribuição de energia e matéria através do tensor energia-momento. De uma maneira mais esquemática, podemos escrever

$$
\underbrace{R_{\mu \nu}-\frac{1}{2} R g_{\mu \nu}+\Lambda g_{\mu \nu}}_{\text {Informa a matéria como se deslocar }}=\underbrace{\kappa T_{\mu \nu}}_{\text {Informa ao espaço-tempo como se curvar }} .
$$

Podemos ver que a equação (84) em quatro dimensões representa 16 equações diferenciais não lineares acopladas. Em decorrência da simetria dos tensores $R_{\mu \nu}$ e $T_{\mu \nu}$ o número de equações diferenciais se reduz para 10. Ainda da equação (84), podemos perceber que que o tensor energia-momento está relacionado de forma linear com a curvatura. 


\subsection{O TENSOR ENERGIA-MOMENTO}

As informações sobre a distribuição de matéria e energia no espaço estão contidas no tensor energia-momento. O tensor $T_{\mu \nu}$ descreve o fluxo de energia e momento existentes na região em análise, e pode assumir diversas formas, a depender do tipo de distribuição desejada.

Um caso de grande relevância no contexto das soluções exatas das equações de campo de Einstein é o vácuo. Para descrever vácuo o tensor energia momento assume a forma (CARROLL, 2004; MISNER; THORNE; WHEELER, 2017)

$$
T_{\mu \nu}=0
$$

Esta distribuição de massa e energia simplifica consideravelmente a resolução das equações de campo, sendo um cenário de grande interesse, além de ser uma ótima aproximação para sistemas como o sistema solar.

Um outro exemplo usual de distribuição é o chamado fluido perfeito. A forma geral covariante deste tensor é dada pela expressão

$$
T_{\mu \nu}=(\rho+p) u_{\mu} u_{\nu}+p g_{\mu \nu}
$$

sendo $\rho$ a densidade de energia, $p$ a pressão e $u$ o campo vetorial associado à velocidade do fluido (WEINBERG; DICKE, 1973).

\subsection{ESPAÇOS-TEMPOS ESFERICAMENTE SIMÉTRICOS}

A realidade dos sistemas físicos é repleta de não linearidades e dinâmicas de grande complexidade, tornando a precisa descrição destes sistemas através de modelos uma árdua tarefa, que na maioria das vezes se mostra infactível. A fim de superar esta dificuldade, aproximações são realizadas e modelos aproximativos são estudados. No campo da relatividade geral o procedimento é semelhante, uma vez que as equações de campo (85) têm natureza não linear. Em geral, para descrever determinados sistemas se assume simetria esférica, o que é uma boa aproximação para o espaço-tempo deformado por um planeta ou estrela.

Definição 3.13. Uma variedade $\mathcal{M}$ possui uma simetria (também chamada de isometria) se a sua geometria é invariante sobre certa transformação que mapeia $\mathcal{M}$ nela mesma. 
Mais especificamente, um espaço-tempo possui simetria se a sua métrica é invariante por uma transformação contínua atuando sobre a variedade.

A simetria pode ser avaliada através de campos vetoriais chamados campos de Killing definidos como segue

Definição 3.14 (Equação de Killing). Dada uma geometria $M$, um campo vetorial de Killing $\mathcal{K}_{\nu}$ é um um campo vetorial que preserva a métrica, ou seja, a métrica permanece inalterada ao longo de seu fluxo.

Os campos de Killing são construídos através da equação de Killing, definida como segue.

Definição 3.15 (Campos de Killing). Os campos $\mathcal{K}_{\nu}$ que são solução da equação de Killing,

$$
\nabla_{\mu} \mathcal{K}_{\nu}+\nabla_{\nu} \mathcal{K}_{\mu}=0
$$

são os campos vetoriais de Killing.

Os campos de Killing geram isometrias, veja por exemplo a figura 7. Como um resultado importante, temos que se a métrica é independente de alguma coordenada $x^{\sigma}$, o vetor $\nabla_{\sigma}$ irá satisfazer a equação de Killing consequentemente.

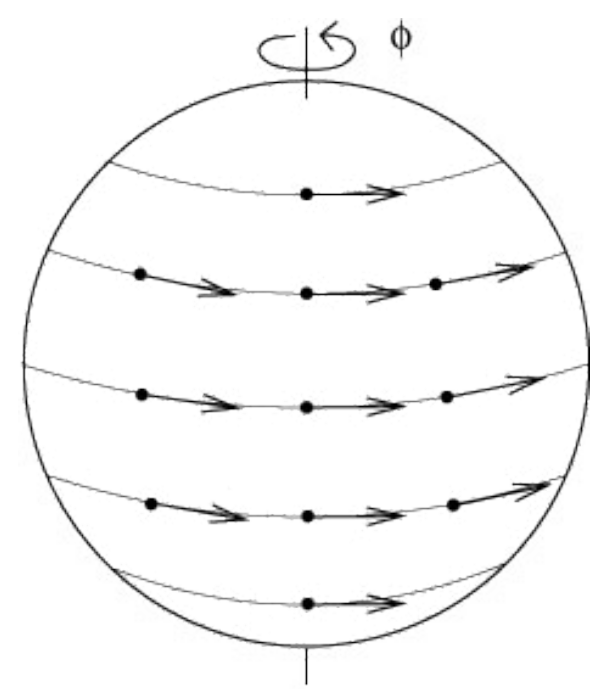

Figura 7 - Campo de Killing associado à coordenada $\phi$. Fonte: Carroll (2018). 


\subsection{EQUAÇÕES DO MOVIMENTO GEODÉSICO EM ESPAÇOS-TEMPOS SIMÉTRICOS}

A classe de geometrias que inicialmente nos interessa são os espaço-tempos estáticos e esfericamente simétricos, esta classe abrange soluções do tipo Schwarzschild e MorrisThorne. Geodésicas tipo tempo descrevem o movimento de partículas massivas, enquanto que geodésicas tipo luz modelam partículas com massa de repouso nula. A dinâmica geodésica pode ser bastante complexa, e mesmo caótica, dada a natureza não-linear das equações de Einstein (CARROLL, 2004; CHANDRASEKHAR, 1998).

Inicialmente, devemos encontrar a Lagrangiana para uma partícula de teste nesta classe de geometrias. Os espaços-tempos com simetria esférica e estáticos possuem o elemento de linha da seguinte forma genérica (CHANDRASEKHAR, 1998),

$$
d s^{2}=g_{\mu \nu} \mathrm{d} x^{\mu} \mathrm{d} x^{\nu}=A(r) \mathrm{d} t^{2}-\frac{1}{B(r)} \mathrm{d} r^{2}-r^{2} \mathrm{~d} \Omega^{2},
$$

onde $\mathrm{d} \Omega^{2}$ é o elemento de linha de uma esfera de raio unitário,

$$
\mathrm{d} \Omega^{2}=\mathrm{d} \theta^{2}+\sin ^{2} \theta \mathrm{d} \phi^{2}
$$

Uma vez que o elemento de linha assume a forma (91), a geodésica pode ser derivada da Lagrangiana (CHANDRASEKHAR, 1998),

$$
2 \mathscr{L}=g_{\mu \nu} \frac{\mathrm{d} x^{\mu}}{\mathrm{d} \lambda} \frac{\mathrm{d} x^{\nu}}{\mathrm{d} \lambda},
$$

onde $\lambda$ é algum parâmetro afim ao longo da geodésica.

Definição 3.16 (Parâmetro afim). Sejam as equações que descrevem o movimento geodésico parametrizadas por $\lambda$. O parâmetro $\lambda$ é chamado de parâmetro afim ${ }^{1}$ se ele torna homogênea a equação da geodésica (WEINBERG; DICKE, 1973),

$$
\frac{\mathrm{d}^{2} x^{\mu}}{\mathrm{d} \lambda^{2}}+\Gamma_{\nu \sigma}^{\mu} \frac{\mathrm{d} x^{\nu}}{\mathrm{d} \lambda} \frac{\mathrm{d} x^{\sigma}}{\mathrm{d} \lambda}=0
$$

Se a conexão for compatível com a métrica, o tensor métrico será transportado paralelamente na geodésica e fornecerá a seguinte Lagrangiana,

$$
2 \mathscr{L}=
$$

$$
A(r(\lambda))\left(\frac{\mathrm{d} t(\lambda)}{\mathrm{d} \lambda}\right)^{2}-\frac{1}{B(r(\lambda))}\left(\frac{\mathrm{d} r(\lambda)}{\mathrm{d} \lambda}\right)^{2}-r(\lambda)^{2}\left(\frac{\mathrm{d} \theta}{\mathrm{d} \lambda}\right)^{2}-r(\lambda)^{2} \sin ^{2} \theta\left(\frac{\mathrm{d} \phi(\lambda)}{\mathrm{d} \lambda}\right)^{2} .
$$

1 O parâmetro afim recebe esse nome por conta que, dados dois parâmetros $\lambda_{1}$ e $\lambda_{2}$ que satisfazem a definição 3.16, então eles se relacionam através de uma função afim $\lambda_{1}=a \lambda_{2}+b$. 
Utilizando a definição canônica de momento apresentada na equação (10),

$$
\frac{\delta \mathscr{L}}{\delta\left(\mathrm{d} q_{\mu} / \mathrm{d} \lambda\right)}=p_{\mu}
$$

que nos fornece para as componentes da métrica (91),

$$
\frac{\delta \mathscr{L}}{\delta \dot{q}_{0}}=p_{t}=A(r) \frac{\mathrm{d} t}{\mathrm{~d} \lambda} .
$$

Em geral, interpreta-se o momento relacionado ao tempo como sendo a energia associada à partícula. Uma vez que o sistema é estacionário, a energia total é uma quantidade que se conserva,

$$
p_{t}=A(r) \frac{\mathrm{d} t}{\mathrm{~d} \lambda}=\text { cte } .
$$

Assim como,

$$
\frac{\delta \mathscr{L}}{\delta \dot{q}_{2}}=p_{\theta}=-r^{2}(\lambda) \frac{\mathrm{d} \theta(\lambda)}{\mathrm{d} \lambda}
$$

$\mathrm{e}$

$$
\frac{\delta \mathscr{L}}{\delta \dot{q}_{3}}=p_{\phi}=-r^{2}(\lambda) \sin ^{2}(\theta) \frac{\mathrm{d} \phi(\lambda)}{\mathrm{d} \lambda},
$$

que são entendidos como as componentes do momento associadas as coordenadas $\theta$ e $\phi$, e

$$
\frac{\delta \mathscr{L}}{\delta \dot{q}_{1}}=p_{r}=-B(r(\lambda))^{-1} \frac{\mathrm{d} r(\lambda)}{\mathrm{d} \lambda}
$$

a componente do momento à trajetória.

Montando a Hamiltoniana a partir da Lagrangiana do problema, chega-se à seguinte equação,

$$
\mathscr{H}=\sum_{\mu=0}^{3} \dot{q}_{\mu} \frac{\partial \mathscr{L}}{\partial \dot{q}_{\mu}}-\mathscr{L}=\sum_{\mu=0}^{3} \dot{q}_{\mu} p_{\mu}-\mathscr{L}
$$

$\mathrm{Ou}$

$$
\begin{aligned}
& \mathscr{H}= \\
& \underbrace{A(r)\left(\frac{\mathrm{d} t}{\mathrm{~d} \lambda}\right)^{2}-\left(B(r)^{-1}\left(\frac{\mathrm{d} r}{\mathrm{~d} \lambda}\right)^{2}+r^{2}\left(\frac{\mathrm{d} \theta}{\mathrm{d} \lambda}\right)^{2}+r^{2} \sin ^{2}(\theta)\left(\frac{\mathrm{d} \phi}{\mathrm{d} \lambda}\right)^{2}\right)}_{=2 \mathscr{L}}-\mathscr{L}=\mathscr{L} .
\end{aligned}
$$

O fato da Lagrangiana ser igual a Hamiltoniana implica não haver energia potencial associada ao problema, apenas energia cinética, o que pode ser observado da Lagrangiana (93) (CHANDRASEKHAR, 1998). Deste fato segue que a Hamiltoniana e, consequentemente, a Lagrangiana serão constantes,

$$
\mathscr{H}=\mathscr{L}=\text { cte }
$$


Realizando um reescalamento do parâmetro afim $\lambda$ é possível colocar $\mathscr{L}=1$ para geodésicas do tipo tempo e $\mathscr{L}=0$ para geodésicas do tipo luz.

Das equações

$$
\begin{aligned}
& \frac{\mathrm{d} p_{t}}{\mathrm{~d} \lambda}=\frac{\partial \mathscr{L}}{\partial t}=0, \\
& \frac{\mathrm{d} p_{\phi}}{\mathrm{d} \lambda}=\frac{\partial \mathscr{L}}{\partial \phi}=0,
\end{aligned}
$$

vemos que as funções $p_{t}$ e $p_{\phi}$ se conservam, assim definimos sem perdas de generalidade,

$$
\begin{aligned}
& p_{t}=E, \\
& p_{\phi}=L .
\end{aligned}
$$

Interpreta-se, respectivamente, a equação (107) como a energia cinética do sistema e a equação (108) como o momento associado à variável angular $\phi$ (momento angular por unidade de massa de repouso) (MISNER; THORNE; WHEELER, 2017).

Resolvendo as quantidades conservadas $E$ e $L$ para os termos diferenciais no plano orbital $\pi / 2$ e substituindo na equação da Lagrangiana temos,

$$
\begin{gathered}
\frac{\mathrm{d} t}{\mathrm{~d} \lambda}=\frac{E}{A(r(\lambda))}, \\
\frac{\mathrm{d} \phi}{\mathrm{d} \lambda}=-\frac{L}{r^{2}(\lambda)}, \\
A(r(\lambda))\left(\frac{E}{A(r(\lambda))}\right)^{2}-\frac{1}{B(r(\lambda))}\left(\frac{\mathrm{d} r(\lambda)}{\mathrm{d} \lambda}\right)^{2}-r^{2}(\lambda)\left(\frac{L}{r^{2}(\lambda)}\right)^{2}=2 \mathscr{L},
\end{gathered}
$$

$\mathrm{com}$

$$
\begin{aligned}
& \mathscr{L}=0 \quad \text { para partículas com massa de repouso nula } \\
& \mathscr{L}=1 \quad \text { para partículas massivas } .
\end{aligned}
$$

Resolvendo para $\dot{r}(\lambda)$, temos,

$$
-2 \mathscr{L}-\frac{1}{B(r(\lambda))}\left(\frac{\mathrm{d} r(\lambda)}{\mathrm{d} \lambda}\right)^{2}=-\frac{E^{2}}{A(r(\lambda))}+\frac{L^{2}}{r^{2}(\lambda)},
$$

multiplicando os dois lados por $-B(r(\lambda)$ ), temos (DÉCANINI; FOLACCI; RAFFAELLI, 2010),

$$
2 \mathscr{L} B(r(\lambda))+\left(\frac{\mathrm{d} r(\lambda)}{\mathrm{d} \lambda}\right)^{2}=\frac{B(r(\lambda))}{A(r(\lambda))} E^{2}-\frac{L^{2}}{r^{2}(\lambda)} B(r(\lambda))
$$




$$
\left(\frac{\mathrm{d} r(\lambda)}{\mathrm{d} \lambda}\right)^{2}=\frac{B(r(\lambda))}{A(r(\lambda))} E^{2}-\frac{L^{2}}{r^{2}(\lambda)} B(r(\lambda))-2 \mathscr{L} B(r(\lambda)) .
$$

Sendo o sistema constituído pelas equações (115), (116) e (117) o nosso sistema de equações diferenciais de interesse,

$$
\begin{gathered}
\left(\frac{\mathrm{d} r(\lambda)}{\mathrm{d} \lambda}\right)^{2}=\frac{B(r(\lambda))}{A(r(\lambda))} E^{2}-B(r(\lambda))\left(2 \mathscr{L}+\frac{L^{2}}{r^{2}(\lambda)}\right), \\
\frac{\mathrm{d} t}{\mathrm{~d} \lambda}=\frac{E}{A(r(\lambda))}, \\
\frac{\mathrm{d} \phi}{\mathrm{d} \lambda}=-\frac{L}{r^{2}(\lambda)}, \\
\theta(\lambda)=\frac{\pi}{2} .
\end{gathered}
$$

\subsection{A SOLUÇÃO DE SCHWARZSCHILD}

Existem diversas soluções para as equações de campo de Einstein, no entanto, uma das mais simples e relevantes foi derivada em 1916 pelo astrônomo e físico alemão Karl Schwarzschild (1873-1916). ${ }^{2}$

As equações de campo de Einstein relacionam a geometria do espaço-tempo com a distribuição de matéria e energia da região em questão. É possível estudar soluções da equação (84) para uma distribuição de vácuo, ou seja, com tensor energia-momento dado pela equação (88). Contraindo ambos os lados da equação (84) e fazendo $\Lambda=0$, é possível verificar que

$$
\underbrace{R_{\mu \nu} g^{\mu \nu}}_{R}-\frac{1}{2} R \underbrace{g_{\mu \nu} g^{\mu \nu}}_{\delta_{\nu}^{\mu}}=\kappa \underbrace{T_{\mu \nu} g^{\mu \nu}}_{T},
$$

que nos fornece em quatro dimensões,

$$
R=-\kappa T
$$

2 Em 1914, a Europa foi abatida pela primeira guerra mundial, fato que fez Karl Schwarzschild servir voluntariamente ao exército alemão, mesmo estando acima de seus 40 anos. No entanto, em 1916 o quadro de sua doença estava acentuadamente agravado, então Karl conseguiu a liberação do serviço militar dando continuidade ao seu tratamento em sua residência. Em 11 de maio de 1916, dois meses após a sua liberação, Karl Schwarzschild faleceu com a idade de 42 anos, sendo enterrado no Cemitério Municipal de Göttingen (Stadtfriedhof de Göttingen) ao lado de grandes nomes da ciência como Max Born, Max von Laue, David Hilbert, Max Planck e Felix Klein (SUHENDRO, 2008). 
Substituindo na equação (85), obtemos diretamente,

$$
R_{\mu \nu}+\frac{1}{2} \kappa T g_{\mu \nu}=\kappa T_{\mu \nu}
$$

que resolvendo para $R_{\mu \nu}$, nos fornece,

$$
R_{\mu \nu}=\kappa T_{\mu \nu}-\frac{1}{2} \kappa T g_{\mu \nu}=\kappa\left(T_{\mu \nu}-\frac{1}{2} T g_{\mu \nu}\right)
$$

Ou seja,

$$
R_{\mu \nu}=\kappa\left(T_{\mu \nu}-\frac{1}{2} T g_{\mu \nu}\right)
$$

portanto, uma vez que o tensor energia-momento é nulo, a equação (123) se reduz à

$$
R_{\mu \nu}=0
$$

Assumindo um campo gravitacional esfericamente simétrico, que é uma boa aproximação para o campo gravitacional gerado pelo Sol ou pela Terra, pode-se obter a trajetória de partículas de teste quando não há forças externas atuando sobre elas. No contexto da relatividade geral, existe um importante teorema que afirma a unicidade de soluções simétricas e estáticas.

Teorema 3.17 (Teorema de Birkhoff ${ }^{3}$ ). Dadas as equações de campo de Einstein (85), qualquer solução de vácuo com simetria esférica e $\Lambda=0$ deve ser descrita pela métrica de Schwarzschild, que em coordenadas esféricas $\{t, r, \theta, \phi\}$ é dada por (SCHUTZ; WILL, 1985; WEINBERG; DICKE, 1973)

$$
\mathrm{d} s^{2}=g_{\mu \nu} \mathrm{d} x^{\mu} \mathrm{d} x^{\nu}=\left(1-\frac{2 G M}{r}\right) \mathrm{d} t^{2}-\left(1-\frac{2 G M}{r}\right)^{-1} \mathrm{~d} r^{2}-r^{2} \mathrm{~d} \Omega^{2},
$$

onde

$$
\mathrm{d} \Omega^{2}=\mathrm{d} \theta^{2}+\sin ^{2} \theta \mathrm{d} \phi^{2},
$$

em que $M$ é a massa do objeto gerador do campo gravitacional e $x^{0}=t, x^{1}=r, x^{2}=$ $\theta, x^{3}=\phi$.

Vemos que a métrica de Schwarzschild é estática, com os campos de Killing,

$$
\mathcal{T}=\frac{\partial}{\partial t}
$$

associado à estaticidade do espaço-tempo e

$$
\mathcal{K}=\frac{\partial}{\partial \phi},
$$

3 George David Birkhoff, 1884-1944. 
associado à simetria em relação à variável angular $\phi$. Fazendo $r \rightarrow \infty$ na métrica de Schwarzschild (125) se obtém a métrica de Minkowski

$$
\mathrm{d} s_{\text {Minkowski }}^{2}=\mathrm{d} t^{2}-\mathrm{d} r^{2}-r^{2} \mathrm{~d} \Omega^{2}
$$

de forma que a geometria de Schwarzschild é assintoticamente plana. A métrica de Minkowski (129) descreve um espaço-tempo regular em todos os pontos, sem matéria e, portanto, sem gravidade.

\subsection{A SOLUÇÃO DE SITTER}

Sejam as equações de Einstein (84) com uma eventual constante cosmológica não nula escrita no sistema natural de unidades. A solução de vácuo $\left(T_{\mu \nu}=0\right)$ e maximalmente simétrica (com 10 campos de Killing linearmente independentes) pode ser escrita na forma do elemento de linha (91) com funções métricas

$$
A(r)=B(r)=1-\frac{\Lambda}{3} r^{2}
$$

Se $\Lambda>0$, temos a métrica de Sitter. Se $\Lambda=0$, temos a métrica de Minkowski. Se $\Lambda<0$, temos a métrica anti-de Sitter.

Para a solução de Sitter, o espaço-tempo associado possui um horizonte em $r=r_{c}$, onde $r_{c}$ é o chamado "raio cosmológico":

$$
r_{c}^{2}=\frac{3}{\Lambda}
$$

O horizonte em $r=r_{c}$ é dito um horizonte cosmológico, porque observadores descritos por curvas tipo tempo ou sinais descritos por curvas tipo luz podem sair do horizonte, mas não podem voltar. Este horizonte permite a passagem em uma única direção. Entretanto, ao contrário do horizonte de eventos no buraco negro de Schwarzchild, tudo sai, e nada entra (KIM; OH; PARK, 2002).

A solução de Sitter é uma solução cosmológica, que descreve um universo homogêneo e isotrópico que se expande exponencialmente. O espaço-tempo associado é, por vezes, chamado de Universo de Sitter (KIM; OH; PARK, 2002). 


\subsection{BURACOS DE MINHOCA ASSINTOTICAMENTE DE SITTER}

Uma geometria que pode ser interpretada como uma estrutura inserida em uma brana com constante cosmológica positiva foi obtida em Molina e Neves (2012a). Neste caso, não há soluções tipo buraco negro. Existem apenas somente soluções descrevendo buracos de minhoca. As equações de campo na brana, generalizando as equações de Einstein quadridimensionais (84), são:

$$
G_{\mu \nu}=-\Lambda g_{\mu \nu}+\kappa^{2} \mathcal{T}_{\mu \nu}+6 \frac{\kappa^{2}}{\lambda} \mathcal{S}_{\mu \nu}-\mathcal{E}_{\mu \nu}+4 \frac{\kappa^{2}}{\lambda} \mathcal{F}_{\mu \nu}
$$

onde $G_{\mu \nu}$ é o tensor de Einstein quadridimensional, $\mathcal{T}_{\mu \nu}$ é o tensor energia-momento na brana, enquanto que $\mathcal{S}_{\mu \nu}$ e $\mathcal{F}_{\mu \nu}$ são termos de correções de alta energia.

Na expressão (132), as correções gravitacionais do bulk sobre a brana são expressas pelo termo $\mathcal{E}_{\mu \nu}$, a projeção do tensor de Weyl 5-dimensional na brana. Este termo depende fortemente da forma do bulk, e não pode ser determinado somente através de informações na brana. Carrega portanto as correções "não-locais" do bulk sobre a brana. A constante cosmológica efetiva na brana é dada por $\Lambda$.

Assumindo vácuo na brana, as equações de campo gravitacionais quadridimensionais se reduzem a

$$
R_{\mu \nu}-\frac{1}{2} R g_{\mu \nu}=-\Lambda g_{\mu \nu}-\mathcal{E}_{\mu \nu}
$$

Uma combinação das equações efetivas (133) escrita sem a especificação de $\mathcal{E}_{\mu \nu}$ é o traço da equação (133):

$$
R=4 \Lambda
$$

onde $R$ denota o escalar de Ricci quadridimensional.

Uma solução não-trivial com $\Lambda>0$ é dada por

$$
\begin{gathered}
A(r)=1-\frac{r^{2}}{r_{c}^{2}}, \\
B(r)=\left(1-\frac{r^{2}}{r_{c}^{2}}\right) \underbrace{\left[1+(C-1) \frac{r_{c}\left(r_{c}^{2}-r_{0}^{2}\right)^{3 / 2}}{r\left(r^{2}-r_{0}^{2}\right)^{3 / 2}}\right]}_{b(r)},
\end{gathered}
$$

onde

$$
r_{c}=\sqrt{\frac{3}{\Lambda}}, r_{0}=\sqrt{\frac{2}{\Lambda}}
$$




$$
0<C<1
$$

Soluções mais complexas podem ser vistas em (MOLINA; NEVES, 2012a). A solução apresentada (wh_dS) descreve um buraco de minhoca, que liga dois horizontes cosmológicos (em $r=r_{c}$ ). A garganta do buraco de minhoca é localizada em $r=r_{g}$, onde

$$
B\left(r_{g}\right)=0
$$

$\mathrm{Ou}$

$$
r_{g}\left(r_{g}^{2}-r_{0}^{2}\right)^{3 / 2}=(1-C) r_{c}\left(r_{c}^{2}-r_{0}^{2}\right)^{3 / 2}
$$

ou ainda

$$
r_{g}\left(r_{g}^{2}-\frac{2}{\Lambda}\right)^{3 / 2}=\frac{\sqrt{3}(1-C)}{\Lambda^{2}} .
$$

Uma restrição sobre os horizontes e a garganta do buraco de minhoca nesta solução é que

$$
r_{0}<r_{g}<r_{c}
$$

O sistema de coordenadas $(t, r, \theta, \phi)$ é válido para $r_{g}<r<r_{c}$.

\subsection{LIMITES EXTREMO E QUASE-EXTREMO DA SOLUÇÃO WH_DS}

O limite quase-extremo é obtido com $C$ próximo de 0 , porém ainda positivo $(C \gtrsim 0)$. Neste caso, a garganta está muito próxima do horizonte cosmológico. Na extensão maximal, os horizontes cosmológicos (dos dois lados da garganta) estão muito próximos. Temos um espaço-tempo onde o horizonte possui gravidade superficial próxima de zero, porém não nula. Definindo o parâmetro adimensional $\delta$ da seguinte forma,

$$
\delta \equiv \frac{r_{c}-r_{g}}{r_{c}}=1-\frac{r_{g}}{r_{c}},
$$

de forma que $0<\delta<1$, é possível manipular algebricamente a expressão (143) para encontrar uma expressão analítica para o raio da garganta,

$$
r_{g}=r_{c}\left(1-\frac{C}{10}\right) .
$$

Realizando uma mudança de coordenadas na equação diferencial (115) para a coordenada radial quase-global u definida por Bronnikov et al. (2008),

$$
\frac{\mathrm{d} u}{\mathrm{~d} r}=\sqrt{\frac{A(r)}{B(r)}}=\frac{1}{\sqrt{b(r)}},
$$


podemos linearizar a função $b(r)$ via série de Taylor em torno do raio da garganta e integrar analiticamente a equação diferencial (145). Como $C \approx 0$, os termos de maior relevância são os lineares, assim, realiza-se também uma expansão em série de MacLaurin em $C$, para obter a seguinte função $r(u)$,

$$
r(u)=r_{g}+\frac{\zeta}{4} u^{2}
$$

com

$$
\zeta \equiv \frac{10}{r_{c}}+\frac{23 C}{5 r_{c}}
$$

É de interesse o cálculo da gravidade superficial nos horizontes, que para esta classe de espaço-tempo pode ser avaliada através da seguinte expressão

$$
\kappa_{r_{0}}=\left|\frac{\mathrm{d}}{\mathrm{d} r}(\sqrt{A(r) B(r)})\right|_{r=r_{0}},
$$

onde $A(r)$ e $B(r)$ são definidos pelo elemento de linha (91) e $r_{0}$ é algum horizonte associado ao espaço-tempo (CARDOSO et al., 2009).

\subsection{A SOLUÇÃO CFM}

Uma solução alternativa para espaços-tempo no contexto das branas foi proposta por Casadio, Fabbri e Mazzacurati (CASADIO; FABBRI; MAZZACURATI, 2002). A solução apresentada encontrada é dada pelo elemento de linha (91) com as funções métricas da seguinte forma,

$$
A(r)=1-\frac{2 M}{r}
$$

e

$$
B(r)=A(r)\left[1+\frac{(C-1) M}{2 r-3 M}\right] .
$$

As soluções definidas pelas funções (149) e (150) são parametrizadas pela constante $C$ (que está relacionado com o parâmetro pós Newtoniano $\beta$ proposto por Casadio, Fabbri e Mazzacurati (2002)), que altera a estrutura do espaço-tempo associado, no entanto, diferentemente da geometria wh_dS, $C$ não possui um limite superior ou inferior. Os possíveis casos de espaços-tempo associados à solução CFM estão esquematizados no quadro 3 . 
Parâmetro $C$

Estrutura

$C<0 \quad$ Buraco de minhoca atravessável

$C=0 \quad$ Buraco negro com horizonte duplo e interior regular

$0<C<1 \quad$ Buraco negro regular sem singularidade interna

$C=1$

Buraco negro de Schwarzschild

$C>1$

Buraco negro com singularidade interna

Quadro 3 - Possíveis casos para a solução CFM em termos do parâmetro $C$.

Fonte: Elaborado pelo autor. 


\section{ANÁLISE DA DINÂMICA GEODÉSICA}

Nesta seção serão estudados os aspectos dinâmicos das geodésicas do tipo tempo e do tipo luz no contexto da geometria de Schwarzschild, do buraco de minhoca assintoticamente de Sitter e CFM. A caracterização de pontos fixos será realizada e sua estabilidade será estudada a partir dos métodos de Lyapunov e Jacobi, bem como eventuais transições de estabilidade.

\subsection{RELAÇÃO ENTRE ESTABILIDADE DE LYAPUNOV E JACOBI}

É possível realizar uma associação entre as noções de estabilidade de Lyapunov e de Jacobi, como apresentadas no capítulo 2, seções 2.7 e 2.8, dada a expressão geral para as geodésicas (115) derivada no capítulo 3, seção 3.5.

Tomando a derivada da expressão (115) com relação ao parâmetro afim $\lambda$ de ambos os lados da equação (240), obtemos a seguinte equação diferencial de segunda ordem

$$
\frac{\mathrm{d}^{2} r}{\mathrm{~d} \lambda^{2}}=\frac{1}{2} \frac{\mathrm{d} f}{\mathrm{~d} r}
$$

onde

$$
f(r) \equiv \frac{B(r)}{A(r)} E^{2}-B(r)\left(2 \mathscr{L}+\frac{L^{2}}{r^{2}}\right) .
$$

Comparando a equação (151) com a expressão geral (35) nos fornece,

$$
G^{1}=-\frac{1}{2} \frac{\mathrm{d} f}{\mathrm{~d} r} .
$$

Avaliando a conexão não linear $N_{j}^{i}=N_{1}^{1}=0$, e consequentemente a conexão de Berwald $G_{j l}^{i}=0$ também. Assim, segue que a única componente não nula do tensor de desvio é,

$$
P_{1}^{1}=-\frac{\partial G^{1}}{\partial x^{1}}=\left.\frac{1}{2} \frac{\mathrm{d}^{2} f}{\mathrm{~d} r^{2}}\right|_{r=r_{*}},
$$

onde $r_{\star}$ é o raio do ponto fixo do sistema. Calculando agora os autovalores da matriz Jacobiana (25) associada ao sistema (151) desacoplado, vemos que

$$
\nu= \pm\left.\sqrt{\frac{1}{2} \frac{\mathrm{d}^{2} f}{\mathrm{~d} r^{2}}}\right|_{r=r_{*}} .
$$

Assim, vemos que a situação de estabilidade ocorre quando $f^{\prime \prime}(r)<0$ e que a situação de instabilidade ocorre quando $f^{\prime \prime}(r)>0$, estando ambos os critérios de estabilidade em concordância, como também constatado por Abolghasem (2012) para o caso da geometria de Schwarzschild. 


\subsection{GEOMETRIA DE SCHWARZSCHILD}

A geometria de Schwarzschild é descrita pela métrica (125), que comparada com a métrica mais geral (91) nos fornece

$$
A(r)=B(r)=1-\frac{2 M}{r} .
$$

As geodésicas do tipo tempo são descritas pela equação (115) com $\mathscr{L}=1 / 2$. Podemos colocar a equação na forma do problema Newtoniano de força central, resultando assim em

$$
\left(\frac{\mathrm{d} r}{\mathrm{~d} \lambda}\right)^{2}=E^{2}-V(r)
$$

com

$$
V(r) \equiv\left(1-\frac{2 M}{r}\right)\left(1+\frac{L^{2}}{r^{2}}\right)
$$

onde a dependência de $r$ no parâmetro afim $\lambda$ foi ocultada por simplificação da notação. Algumas curvas do potencial efetivo $V(r)$ são apresentadas no gráfico da figura 8.

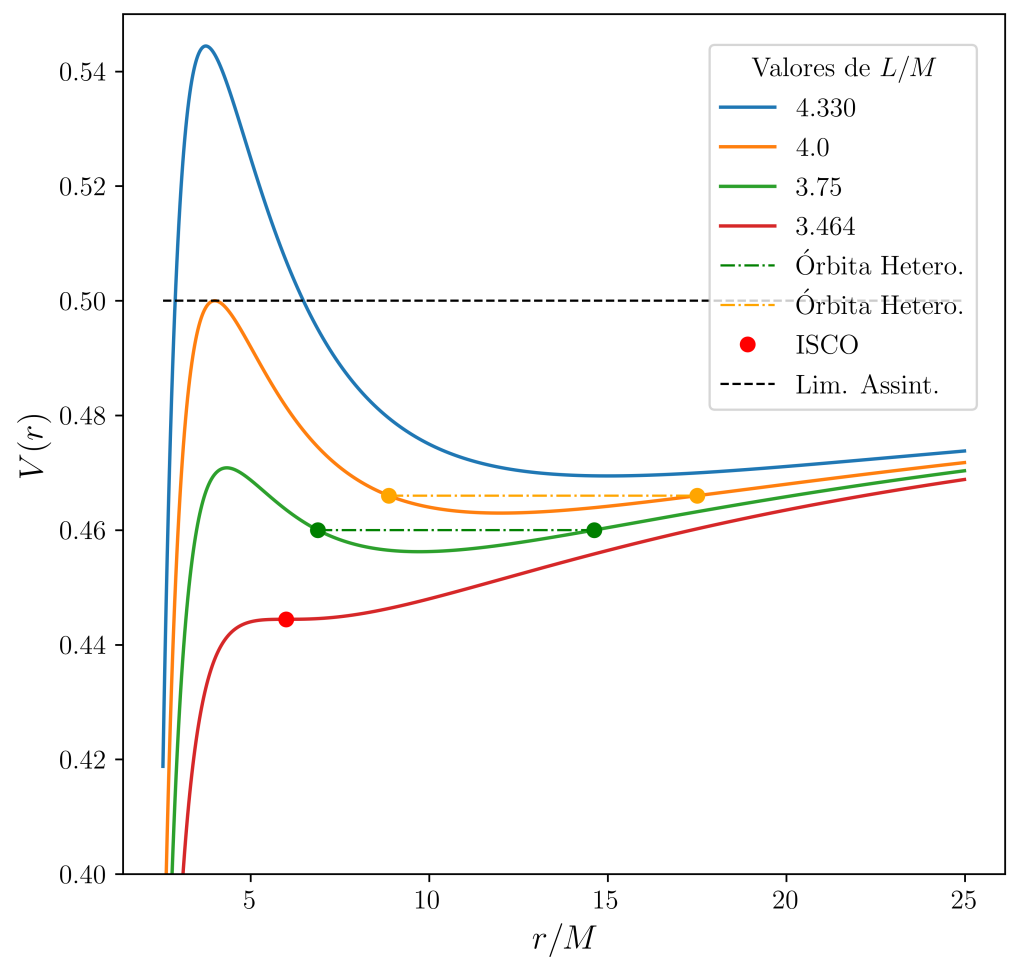

Figura 8 - Curvas para o potencial de Schwarzschild $V(r)$ associado à geodésicas do tipo tempo com vários valores de $L / M$.

Fonte: Elaborado pelo autor.

A partir da figura 8 é possível observar algumas característica qualitativas do sistema. O primeiro fato a ser notado é a existência de dois tipos de órbitas fechadas, as 
perfeitamente circulares com $r$ constante e as elípticas, isto é, órbitas que oscilam entre dois valores extremos $r_{1} \leq r \leq r_{2}$ que são determinados pela energia total do sistema E. Estes valores extremos representam pontos fixos da equação diferencial (157) e estas órbitas são ditas heteroclínicas.

As órbitas fechadas são elípticas e seus afélio e periélio são seus pontos fixos. Exatamente sobre mínimo do potencial $V(r)$ existe uma órbita circular (de excentricidade zero), que por sua vez é uma raiz de multiplicidade algébrica 2 quando verifica a condição,

$$
V^{\prime}(r)=0
$$

assim temos,

$$
r_{1,2}=\frac{L^{2} \pm \sqrt{L^{4}-12 M^{2} L^{2}}}{2 M} .
$$

Para que a raiz tenha multiplicidade algébrica igual 2 é necessário impor que

$$
L^{2}=12 M^{2}
$$

Substituindo a expressão (161) na equação (160), verifica-se

$$
r=\frac{12 M^{2}}{2 M}=6 M
$$

que é o raio onde ocorre a última órbita estável, ponto que é comumente retratado na literatura pertinente como ISCO (CORNISH; LEVIN, 2003; CHANDRASEKHAR, 1998).

Definindo a seguinte quantidade,

$$
\left(\frac{\mathrm{d} r}{\mathrm{~d} \lambda}\right)^{2} \equiv w^{2}
$$

podemos rearranjar a expressão (157) obter a equação

$$
\frac{w^{2}}{E^{2}}+\frac{V(r)}{E^{2}}=1,
$$

que descreve elipses do espaço de estados do sistema, conforme apresentado na figura 9. 


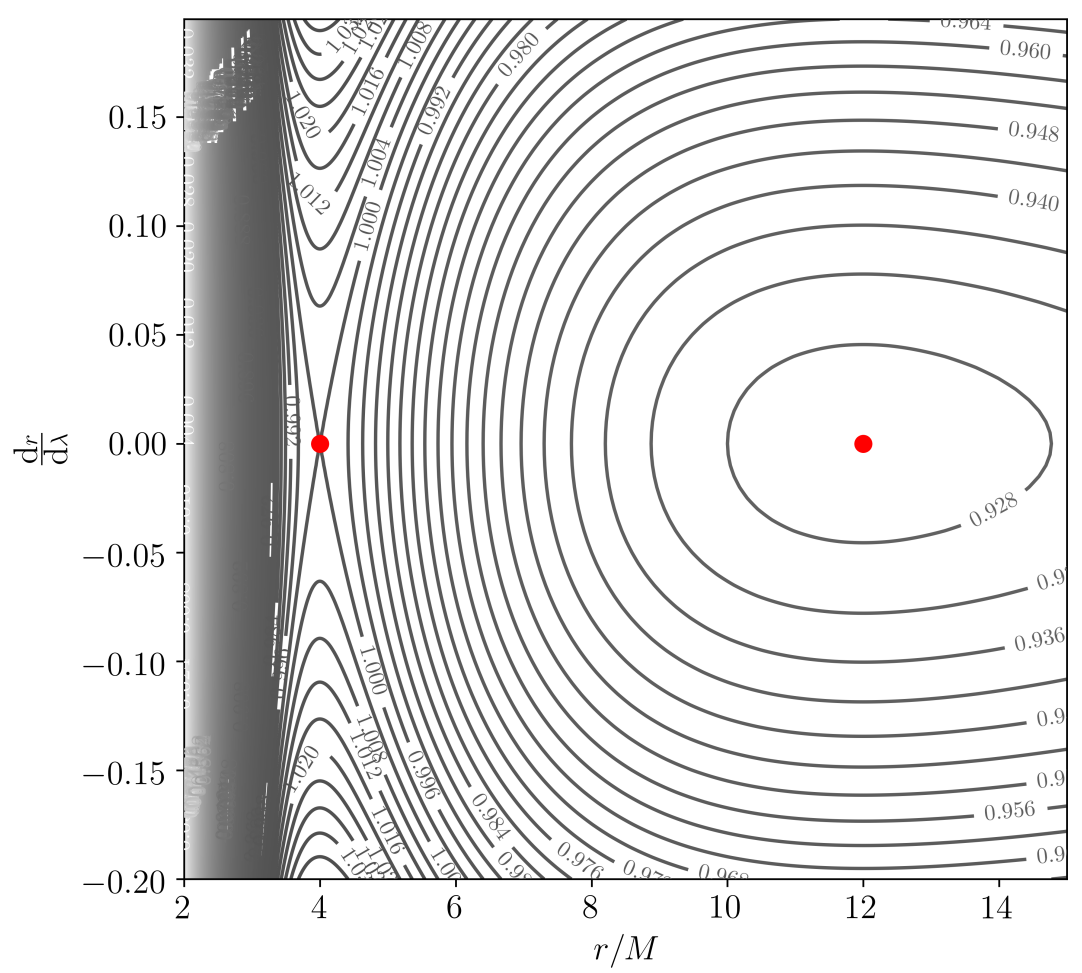

Figura 9 - Espaço de estados para o sistema com $L / M=4$ para diversos valores de $E$. Fonte: Abolghasem (2013).

A fim de estudar a estabilidade dos pontos fixos do sistema deriva-se a equação (157) com relação ao parâmetro afim $\lambda$,

$$
\frac{\mathrm{d}}{\mathrm{d} \lambda}\left(\frac{\mathrm{d} r}{\mathrm{~d} \lambda}\right)^{2}=\frac{\mathrm{d}}{\mathrm{d} \lambda}\left[E^{2}-V(r(\lambda))\right]
$$

que nos fornece pela regra da cadeia,

$$
\frac{\mathrm{d}^{2} r}{\mathrm{~d} \lambda^{2}}=-\frac{1}{2} \frac{\mathrm{d} V}{\mathrm{~d} r}
$$

que é a equação para o método utilizado em (ABOLGHASEM, 2013).

Desacoplando o sistema a fim de reduzir o grau da equação diferencial, temos o seguinte sistema de equações diferenciais,

$$
\begin{aligned}
\dot{r} & =w, \\
\dot{w} & =-\frac{1}{2} \frac{\mathrm{d} V}{\mathrm{~d} r},
\end{aligned}
$$

do qual podemos construir o espaço $(r, w)$. Uma vez que o sistema é Hamiltoniano e o teorema de Liouville assegura a conservação do espaço de estados, as trajetórias parametrizadas por $\lambda$ seguirão curvas $\lambda \rightarrow(r(\lambda), w(\lambda))$ sobre o plano $(r, w)$. A equação de restrição é fornecida por

$$
E^{2}=w^{2}+V(r)=\text { constante },
$$


Para compreender o comportamento assintótico do sistema é necessária a linearização do sistema, e para tal, consideramos que o sistema em questão é duas vezes continuamente diferenciável e que existem finitos pontos de equilíbrio. O campo vetorial $\mathbf{f}: \mathbb{R}_{\geq 0} \times \mathbb{R} \rightarrow \mathbb{R}^{2}$ é definido por

$$
\mathbf{f}(r, w)=(\dot{r}, \dot{w})=\left(w,-1 / 2 V^{\prime}(r)\right)
$$

para o nosso sistema de equações (167) e (168). Os pontos de equilíbrio para este sistema são $\left(r_{*}, 0\right)$, onde $r_{*}$ é o valor do raio onde o potencial $V^{\prime}(r)=0$, ou seja, o raio crítico do potencial. Neste ponto a energia potencial do sistema é um extremo.

A matriz Jacobiana do sistema (168) é dada por

$$
\mathbf{J}=\frac{\partial \mathbf{f}}{\partial \mathbf{x}}(r, w)=\left[\begin{array}{cc}
0 & 1 \\
-\frac{1}{2} V^{\prime \prime}(r) & 0
\end{array}\right]
$$

cuja equação característica é

$$
\operatorname{det}(\mathbf{J}-\nu \mathbf{I})=\nu^{2}+\frac{1}{2} V^{\prime \prime}(r)=0 .
$$

A quantidade $\nu$ representa os autovalores da matriz (171), com

$$
\nu= \pm\left.\sqrt{-\frac{1}{2} V^{\prime \prime}(r)}\right|_{r=r_{\star}} .
$$

Pela classificação dos pontos fixos a partir dos autovalores contidos na tabela 1, podemos inferir as condições associadas a cada possibilidade de ponto fixo.

A primeira possibilidade acontece quando $V^{\prime \prime}\left(r_{*}\right)<0$, nesta situação temos $\nu_{1}<$ $0<\nu_{2} \in \mathbb{R}$, sendo assim um ponto de sela, isto o ocorre quando $V$ possui um máximo local em $r_{*}$. Caso $V^{\prime \prime}\left(r_{*}\right)>0$, temos $\nu_{1}, \nu_{2} \in \mathbb{C}$ e este ponto no espaço de estados será um centro pela conservação da energia total (169), esta condição é satisfeita caso haja um mínimo local de $V(r)$ em $r_{*}$. Quando há $V^{\prime \prime}(r)=0$, há um ponto de inflexão no potencial, então a análise deve ser mais cautelosa.

Para estudar as geodésicas nulas na geometria de Schwarzschild devemos fazer $\mathscr{L}=0$ nas equações que descrevem as geodésicas em geometrias esfericamente simétricas (115)-(118), o que nos fornece o seguinte sistema de equações diferenciais ordinárias,

$$
\begin{gathered}
\left(\frac{\mathrm{d} r}{\mathrm{~d} \lambda}\right)^{2}=E^{2}-\left(1-\frac{2 M}{r}\right) \frac{L^{2}}{r^{2}}, \\
\frac{\mathrm{d} \phi}{\mathrm{d} \lambda}=-\frac{L}{r^{2}},
\end{gathered}
$$




$$
\begin{gathered}
\frac{\mathrm{d} t}{\mathrm{~d} \lambda}=\frac{E}{\left(1-\frac{2 M}{r}\right)}, \\
\theta(\lambda)=\frac{\pi}{2} .
\end{gathered}
$$

O potencial efetivo das geodésicas do tipo luz associado à equação (174) assume a forma,

$$
V(r) \equiv \frac{1}{2}\left(1-\frac{2 M}{r}\right) \frac{L^{2}}{r^{2}}
$$

As soluções de equilíbrio deste sistema dinâmico são os valores de $r$ tais que

$$
E^{2}=V(r)
$$

conforme a equação (157). Podemos observar esta condição graficamente na figura 10a.

Desta forma, observa-se que existem dois pontos fixos reais conforme é variado o valor de $E$, até que se atinge o ponto de inflexão do potencial, onde os dois pontos fixos se encontram no ponto de extremo do potencial. Neste ponto há apenas um ponto fixo de multiplicidade algébrica dois. Este ponto descreve uma geodésica circular, na chamada fotosfera ${ }^{1}$.

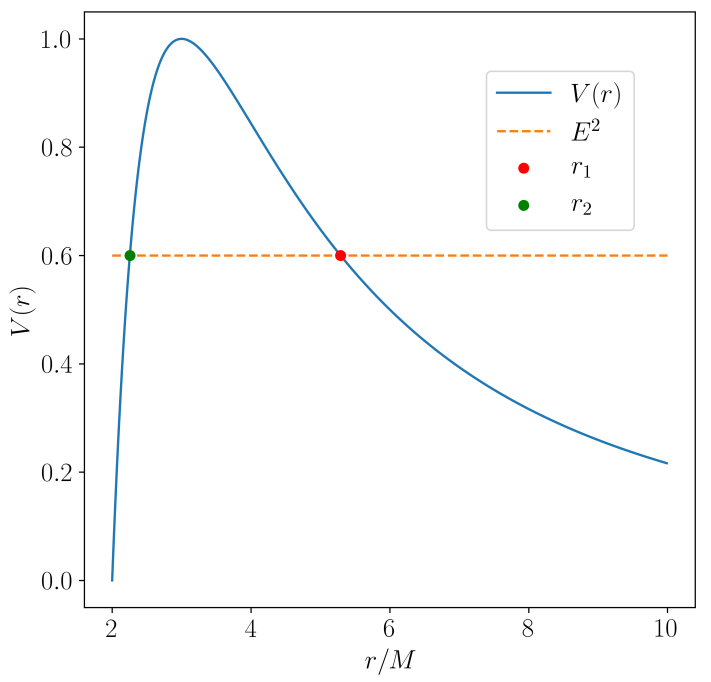

(a)

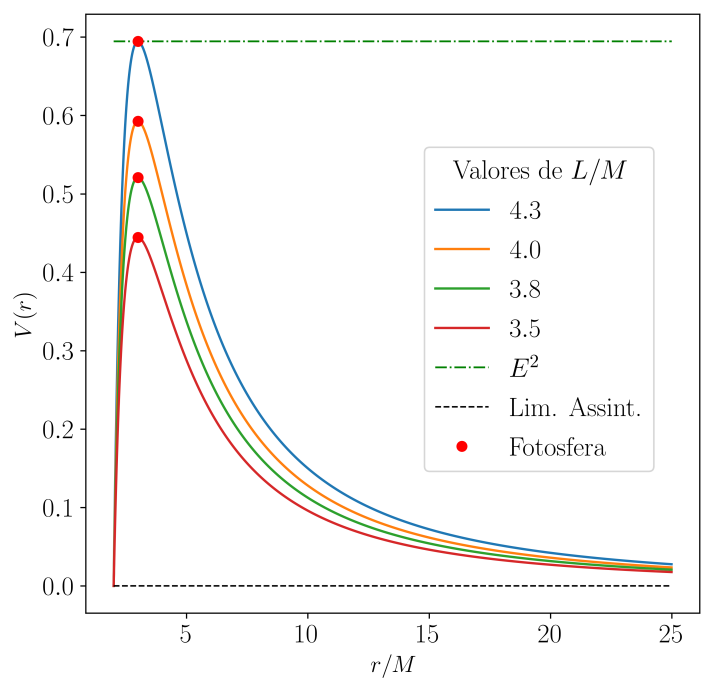

(b)

Figura 10 - Potenciais de Schwarzschild para geodésicas do tipo luz.

Fonte: Elaborado pelo autor.

Na figura 10a está diagramado o potencial para geodésicas do tipo luz, com os pontos indicando na figura os pontos fixos do sistema fixado um valor $E$ de energia. $\mathrm{Na}$

1 Também chamada de esfera de fótons ou, quando na literatura estrangeira, photon sphere ou ainda de lightsphere. 
figura 10b, diversos potenciais para geodésicas do tipo luz explicitando as fotosferas (pontos de equilíbrio dos potenciais correspondentes). O valor do raio da fotosfera $r_{\text {fóton }}$ pode ser encontrado derivando-se o potencial $V(r)$ e igualando-a a zero,

$$
\frac{\mathrm{d} V}{\mathrm{~d} r}=\frac{2 L^{2}(3 M-r)}{r^{4}}=0
$$

de onde podemos perceber que única raiz possível é $r_{\text {fóton }}=3 M$.

O valor crítico de energia para que se atinja este ponto pode ser encontrado a partir da equação (179),

$$
\begin{gathered}
E^{2}=V\left(r_{\text {fóton }}\right)=V(3 M)=\frac{L^{2}}{27 M^{2}}, \\
E=\sqrt{\frac{L^{2}}{27 M^{2}}}= \pm \frac{L}{3 \sqrt{3} M} .
\end{gathered}
$$

Define-se parâmetro de impacto $D$ como segue em 4.1 .

Definição 4.1 (Parâmetro de impacto). Dada uma trajetória $\xi$, o parâmetro de impacto é definido como a distância perpendicular entre a trajetória $\xi$ da partícula e o centro do potencial (MISNER; THORNE; WHEELER, 2017)), expressa como a quantidade,

$$
D \equiv \frac{L}{E}
$$

O parâmetro de impacto crítico para as órbitas do tipo luz é dado por,

$$
D_{c}=3 \sqrt{3} M
$$

Os pontos de fixos $r_{1}$ e $r_{2}$ da figura 10a originam órbitas que são instáveis. Órbitas originadas na periferia do ponto $r_{1}$ caem inevitavelmente no horizonte de eventos, enquanto as órbitas originadas em $r_{2}$ caminham em direção ao infinito.

A análise realizada para estabilidade das trajetórias do tipo tempo se mantém para as trajetórias do tipo luz, sendo a estabilidade de um ponto fixo inferida a partir dos possíveis valores de $\nu$ obtidos pela expressão (173).

A única possibilidade de estabilidade acontece quando $V^{\prime \prime}\left(r_{\text {foton }}\right)<0$, nesta situação temos $\nu_{1}<0<\nu_{2} \in \mathbb{R}$, sendo assim um ponto de sela, isto o ocorre quando $V$ possui um máximo local em $r_{\text {foton }}$. Como o potencial $V(r)$ não admite mínimos locais, não há a possibilidade de ocorrência de centros. Assim, no contexto de partículas não massivas 
temos que em $r=3 M$ ocorre um máximo do potencial, cuja a segunda derivada é dada por,

$$
V^{\prime \prime}(r)=-\frac{6 L^{2}(4 M-r)}{r^{5}} .
$$

Avaliando $V^{\prime \prime}(r)$ em $r=3 M$, temos

$$
V^{\prime \prime}(3 M)=-\frac{2 L^{2}}{81 M^{4}}<0 \quad \forall \quad L \in \mathbb{R} \quad \text { e } \quad M \in \mathbb{R}^{+} .
$$

Desta forma, qualquer geodésica nula que se aproxima da região da fotosfera tende a se afastar dela, ou eventualmente cair no horizonte de eventos em $r=2 M$. O espaço de estados do sistema (168) está plotado na figura 11.

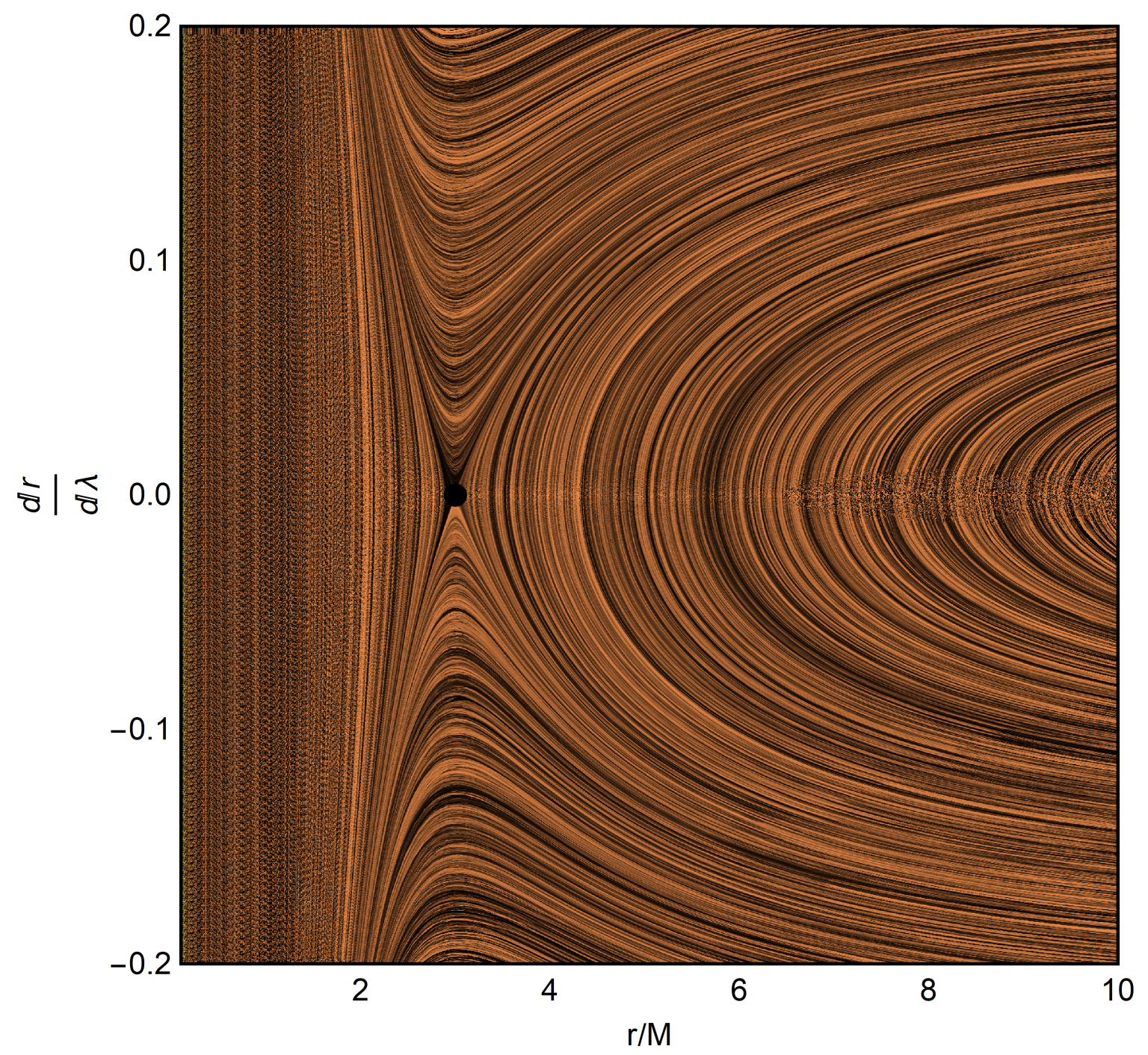

Figura 11 - Espaço de estados gerado via integral de linha convolucional do sistema definido por (167)-(168).

Fonte: Elaborado pelo autor.

No espaço de estados da figura 11 é possível observar que existe um ponto fixo em $r=3 M$, que é o raio da fotosfera. Este ponto é uma sela instável, é possível identificar 
esta instabilidade observando o fluxo das trajetórias vizinhas a este ponto no diagrama 11, que se afastam.

Uma condição para a existência de ciclos limites em um sistema dinâmico é a existência de órbitas fechadas no sistema, que pode ser verificada com o auxílio do teorema 2.16, como veremos a seguir. Assim deseja-se demonstrar a existência de órbitas fechadas para o sistema seja para o caso de geodésicas do tipo tempo ou do tipo luz. Para as trajetórias do tipo tempo em Schwarzschild, são conhecidas as órbitas fechadas do sistema (CORNISH; LEVIN, 2003; CHANDRASEKHAR, 1998), como por exemplo a ISCO, enquanto que para as trajetórias do tipo luz sabe-se que a única trajetória fechada é a fotosfera (CARDOSO et al., 2009). Desta forma, a fim de verificar a impossibilidade de órbitas circulares no sistema, é conveniente transferir o sistema de coordenadas do problema para o plano cartesiano. Para isso consideremos as novas coordenadas $x(\theta(\lambda))$ e $y(\phi(\lambda))$, relacionadas com as coordenadas esféricas por

$$
\begin{aligned}
x(\phi(\lambda)) & =r(\phi(\lambda)) \cos (\phi(\lambda)), \\
y(\phi(\lambda)) & =r(\phi(\lambda)) \sin (\phi(\lambda)), \\
r^{2}(\phi(\lambda)) & =x(\phi(\lambda))^{2}+y(\phi(\lambda))^{2} .
\end{aligned}
$$

Diferenciando as equações (187) e (188) com relação ao parâmetro afim $\lambda$ obtemos o novo sistema de equações,

$$
\begin{aligned}
& \frac{\mathrm{d} x}{\mathrm{~d} \lambda}=\frac{\mathrm{d} r}{\mathrm{~d} \phi} \frac{\mathrm{d} \phi}{\mathrm{d} \lambda} \cos (\phi(\lambda))-r(\phi(\lambda)) \sin (\phi(\lambda)) \frac{\mathrm{d} \phi}{\mathrm{d} \lambda}, \\
& \frac{\mathrm{d} y}{\mathrm{~d} \lambda}=\frac{\mathrm{d} r}{\mathrm{~d} \phi} \frac{\mathrm{d} \phi}{\mathrm{d} \lambda} \sin (\phi(\lambda))+r(\phi(\lambda)) \cos (\phi(\lambda)) \frac{\mathrm{d} \phi}{\mathrm{d} \lambda} .
\end{aligned}
$$

Tendo em mente as relações expressas nas equações (187), (188) e (189), coloca-se o sistema na seguinte forma,

$$
\begin{aligned}
& \frac{\mathrm{d} x}{\mathrm{~d} \lambda}=\frac{\mathrm{d} \phi}{\mathrm{d} \lambda}\left[\frac{x(\lambda)}{\sqrt{x(\lambda)^{2}+y(\lambda)^{2}}} \frac{\mathrm{d} r}{\mathrm{~d} \phi}-y(\lambda)\right], \\
& \frac{\mathrm{d} y}{\mathrm{~d} \lambda}=\frac{\mathrm{d} \phi}{\mathrm{d} \lambda}\left[\frac{y(\lambda)}{\sqrt{x(\lambda)^{2}+y(\lambda)^{2}}} \frac{\mathrm{d} r}{\mathrm{~d} \phi}+x(\lambda)\right],
\end{aligned}
$$

que pela regra da cadeia fica

$$
\begin{aligned}
& \frac{\mathrm{d} x}{\mathrm{~d} \lambda}=\frac{\mathrm{d} \phi}{\mathrm{d} \lambda}\left[\frac{x(\lambda)}{\sqrt{x(\lambda)^{2}+y(\lambda)^{2}}} \frac{\mathrm{d} r}{\mathrm{~d} \lambda} \frac{\mathrm{d} \lambda}{\mathrm{d} \phi}-y(\lambda)\right], \\
& \frac{\mathrm{d} y}{\mathrm{~d} \lambda}=\frac{\mathrm{d} \phi}{\mathrm{d} \lambda}\left[\frac{y(\lambda)}{\sqrt{x(\lambda)^{2}+y(\lambda)^{2}}} \frac{\mathrm{d} r}{\mathrm{~d} \lambda} \frac{\mathrm{d} \lambda}{\mathrm{d} \phi}+x(\lambda)\right] .
\end{aligned}
$$


O sistema de equações (194)- (195) não possui ponto fixo, tendo uma descontinuidade em $(x, y)=(0,0)$. Assim, testamos o critério de Bendixson (Teorema 2.16), onde verifica-se que as derivadas parciais são,

$$
\frac{\partial f}{\partial x}=\frac{\partial g}{\partial y}=\frac{\mathrm{d} \phi}{\mathrm{d} \lambda}\left[\frac{1}{r} \frac{\mathrm{d} r}{\mathrm{~d} \lambda} \frac{\mathrm{d} \lambda}{\mathrm{d} \phi}\right]=\frac{1}{r} \frac{\mathrm{d} r}{\mathrm{~d} \lambda},
$$

assim, a soma

$$
\frac{\partial f}{\partial x}+\frac{\partial g}{\partial y}=\frac{2}{r} \frac{\mathrm{d} r}{\mathrm{~d} \lambda} \neq 0 \forall D<3 \sqrt{3} M
$$

Logo, para valores do parâmetro de impacto menores do que o crítico o sistema não admite geodésicas nulas fechadas.

Como discutido na seção 2.2, definição 2.6, uma órbita é homoclínica quando existe uma variedade estável e outra instável associada ela, ambas conectadas ao mesmo ponto fixo do espaço de estados. Para órbitas do tipo tempo, estas duas variedades se interceptam em um ponto, que no contexto de Schwarzschild é a ISCO (CORNISH; LEVIN, 2003).

A ISCO é um ponto fixo do tipo sela, onde as duas variedades estável e instável coexistem, representando assim a divisão entre a estabilidade e a instabilidade do sistema. No caso das geodésicas do tipo tempo de Schwarzschild, as órbitas homoclínicas se iniciam na variedade instável do ponto de sela até atingir um raio máximo, diga-se um $r=r_{\max }$, momento que órbita retorna para a variedade estável. Este deslocamento é observável no espaço de fases do sistema. Um prolongamento de órbita homoclínica para trajetórias do tipo tempo é apresentado na figura 12. 


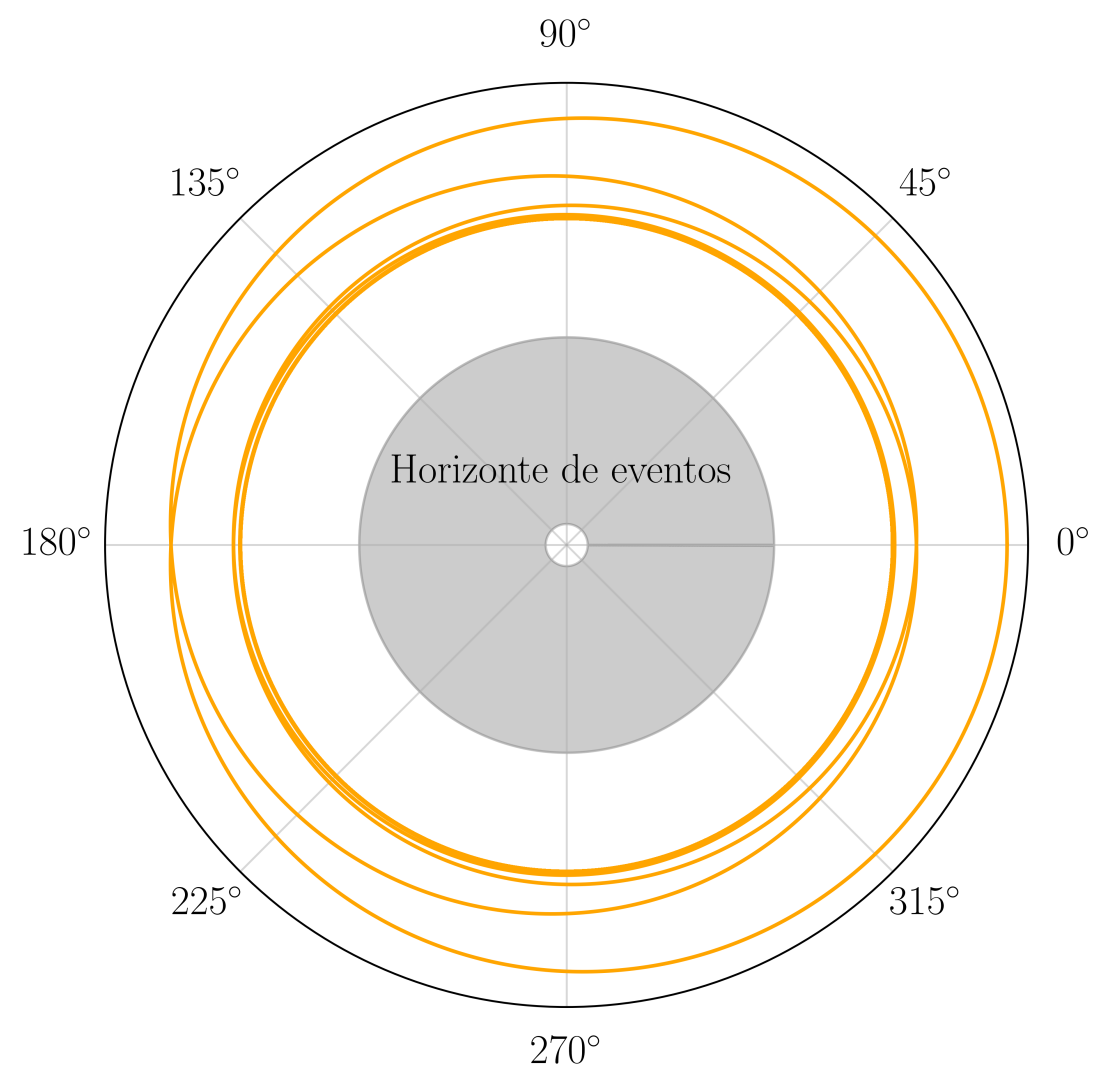

Figura 12 - Trecho de órbita homoclínica (plano orbital) para partículas massivas com $L / M=12 \sqrt{3 / 35}$.

Fonte: Elaborado pelo autor.

Por outro lado, as geodésicas do tipo luz não possuem órbitas homoclínicas em seu espaço de estados uma vez que as órbitas que partem do ponto de sela ou se dirigem ao infinito ou caem no horizonte de eventos, como pode ser observado no espaço de estados da figura 11. No entanto, ao reparametrizar a órbita no parâmetro $\phi$ fornecido pela equação $(175)$, temos $^{2}$,

$$
\frac{\mathrm{d} \phi}{\mathrm{d} \lambda}=-\frac{L}{r^{2}}
$$

e ainda realizando a transformação da variável dinâmica $x \equiv M / r$, a equação (174) se torna

$$
\left(\frac{\mathrm{d} x}{\mathrm{~d} \phi}\right)^{2}=\frac{M^{2} E^{2}}{L^{2}}-x^{2}(1-2 x) .
$$

Através de uma separação de variáveis na equação (199), temos a seguinte integração,

$$
\int \mathrm{d} \phi=\int \frac{\mathrm{d} x}{\sqrt{P(x)}},
$$

2 Método apresentado em Chandrasekhar (1998, p. 123). 
onde

$$
P(x)=\frac{M^{2} E^{2}}{L^{2}}-x^{2}(1-2 x) .
$$

A integral do lado esquerdo da equação (200) nos fornece $\phi+C$, onde $C$ é uma constante de integração. Por outro lado, a integral do lado direito da equação não é trivial. Trata-se de uma integral elíptica cuja solução é um pouco mais complicada. Colocando o parâmetro $M^{2} E^{2} / L^{2}=1 / 27$, temos que o polinômio $P(x)$ de (200) se torna,

$$
P(x)=\frac{1}{27}-x^{2}(1-2 x)
$$

cujas raízes satisfazem a equação $1 / 27-r^{2}(1-2 r)=0$. O polinômio $P(x)$ possui uma raiz $r_{1}=-1 / 6$ e outras duas iguais (multiplicidade algébrica dois) $r_{2}=r_{3}=1 / 3$, desta forma, podemos reescrevê-lo da seguinte forma

$$
P(x)=2\left(x+\frac{1}{6}\right)\left(x-\frac{1}{3}\right)^{2}
$$

Substituindo o polinômio (203) na equação (200), temos

$$
\phi+C=\int \frac{\mathrm{d} x}{\sqrt{\left(2 x+\frac{1}{3}\right)\left(x-\frac{1}{3}\right)^{2}}} .
$$

A integral (204) pode ser expressa por funções elementares, e resolvida utilizando a substituição padrão

$$
2 x+\frac{1}{3} \equiv t^{2}
$$

cujo o diferencial associado é

$$
\frac{\mathrm{d} t}{\mathrm{~d} x}=\left(2 x+\frac{1}{3}\right)^{-\frac{1}{2}} .
$$

A partir da nossa substituição (205), podemos verificar que $x=t^{2} / 2-1 / 6$, assim, podemos eliminar a variável $x$ da equação,

$$
\phi+C=-2 \int \frac{\mathrm{d} t}{\left(1-t^{2}\right)} .
$$

Tendo em mente que a derivada da função inversa da tangente hiperbólica é dado por,

$$
\frac{\mathrm{d}}{\mathrm{d} x}\left[\tanh ^{-1}(x)\right]=\frac{1}{1-x^{2}},
$$

é possível observar que a primitiva do integrando em (207), é $\tanh ^{-1}(t)$, nos fornecendo,

$$
\phi+C=-2 \tanh ^{-1}\left(\sqrt{2 x+\frac{1}{3}}\right) .
$$


Resolvendo para $x(\phi)$, temos,

$$
x_{\text {out }}(\phi)=\frac{1}{2} \tanh ^{2}\left(\frac{\phi+\phi_{0}}{2}\right)-\frac{1}{6} .
$$

Para uma partícula oriunda do infinito, temos que a condição inicial da trajetória deve satisfazer

$$
\tanh ^{2}\left(\frac{1}{2} \phi_{0}\right)=\frac{1}{3}
$$

o que faz com que $x_{\text {out }}(0)=0$ e pela definição da variável dinâmica $x$ pode-se observar que a partícula vem de $r=\infty$.

Tomando o limite,

$$
\lim _{\phi \rightarrow \pm \infty} x_{\text {out }}(\phi)=\frac{1}{3}
$$

onde, pela definição da variável angular $\phi$ em (175), vemos que $\phi \rightarrow-\infty$ representa uma órbita com momento angular $L<0$ e $\phi \rightarrow \infty$ representa uma órbita com momento angular $L>0$. Observa-se pelo limite (212) que estas órbitas tendem assintoticamente para a fotosfera. A figura 13 mostra as geodésicas do tipo luz com parâmetro de impacto $D_{c}$ crítico nas redondezas da fotosfera.

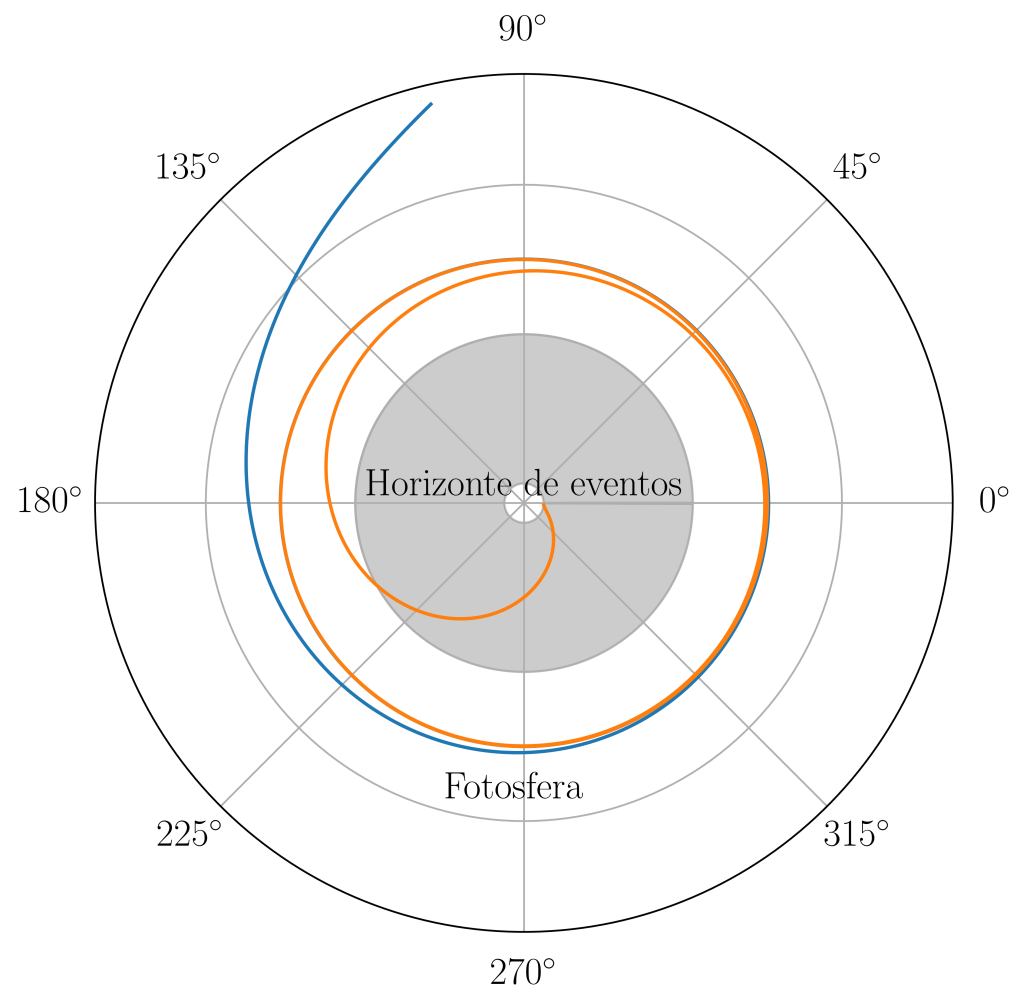

Figura 13 - Geodésicas nulas com $D=D_{c}$.

Fonte: Elaborado pelo autor.

Nota-se que trajetórias vindas de $r=\infty$ tendem assintoticamente à fotosfera, assim como as órbitas originadas no horizonte de eventos em $r=2 M$. As órbitas originadas no 
horizonte de eventos podem ser consideradas órbitas prolongamentos das órbitas vindas de fora da fotosfera, ou seja, órbitas que acabam escapando da região da fotosfera devido algum pequeno desvio sofrido (CHANDRASEKHAR, 1998).

As geodésicas nulas críticas que se iniciam no horizonte de eventos em direção à fotosfera possuem a seguinte solução,

$$
x_{\text {in }}(\phi)=\frac{1}{3}+\frac{2 e^{\phi}}{\left(e^{\phi}-1\right)^{2}},
$$

que ao tomarmos o limite,

$$
\lim _{\phi \rightarrow \pm \infty} x_{\text {in }}(\phi)=\lim _{\phi \rightarrow \pm \infty} \frac{1}{3}+\lim _{\phi \rightarrow \pm \infty} \frac{2 e^{\phi}}{\left[e^{\phi}\left(1-\frac{1}{e^{\phi}}\right)\right]^{2}}=\frac{1}{3},
$$

novamente tendendo assintoticamente à fotosfera. Temos para as órbitas iniciadas do lado de fora da fotosfera,

$$
w_{\text {out }}(\phi)=\frac{\mathrm{d} x_{\text {out }}}{\mathrm{d} \phi}(\phi)=\frac{1}{2} \tanh \left(\frac{\phi+\phi_{0}}{2}\right) \operatorname{sech}^{2}\left(\frac{\phi+\phi_{0}}{2}\right),
$$

cujo limite assimptótico nos fornece,

$$
\lim _{\phi \rightarrow \pm \infty} w_{\text {out }}(\phi)=0
$$

A velocidade radial associada às geodésicas que se iniciam na região interna da fotosfera é dado pela seguinte expressão,

$$
w_{\mathrm{in}}(\phi)=\frac{\mathrm{d} x_{\mathrm{in}}}{\mathrm{d} \phi}(\phi)=-\frac{2 e^{\phi}\left(1+e^{\phi}\right)}{\left(e^{\phi}-1\right)^{3}}
$$

cujo limite fornece,

$$
\lim _{\phi \rightarrow \pm \infty} w_{\text {in }}(\phi)=0 .
$$

O fato das velocidades associadas à coordenada radial tenderem a zero indica que conforme a trajetória se aproxima cada vez mais de $3 M$, menos o raio varia, no entanto estas trajetórias nunca atingem $r=3 M$. Isso indica que a fotosfera é uma órbita fechada e isolada no espaço físico para aquelas partículas que possuem o parâmetro impacto crítico, uma condição necessária para a existência de um ciclo limite. O gráfico 13 junto com os resultados analíticos desta seção mostram que a órbita fechada em $r=3 M$ é um ciclo limite do sistema no plano orbital, de forma que todas as trajetória nulas com parâmetro de impacto crítico tendem assintoticamente à fotosfera.

A estabilidade da fotosfera pode ser estudada através dos métodos de Lyapunov, seja direto ou indireto. Inicialmente, tomemos o método indireto de Lyapunov. Derivando 
ambos os lados da equação (115), podemos construir a seguinte equação envolvendo derivadas primeiras e segundas,

$$
2 \frac{\mathrm{d} r}{\mathrm{~d} \lambda} \frac{\mathrm{d}^{2} r}{\mathrm{~d} \lambda^{2}}=\frac{\mathrm{d} f}{\mathrm{~d} r} \frac{\mathrm{d} r}{\mathrm{~d} \lambda}
$$

A partir da equação (219), temos o seguinte sistema desacoplado que define um campo vetorial em $\mathbb{R}^{2}$,

$$
\vec{z}(r, w) \equiv(\dot{r}, \dot{w})=w \hat{r}+\frac{1}{2} f^{\prime}(r) \hat{w},
$$

onde um ponto indica diferenciação com respeito à $\lambda$ e uma linha com respeito à $r$. Podemos tomar como função de Lyapunov

$$
L(r, w)=\frac{w^{2}}{2}-\frac{f(r)}{2}
$$

e avaliar o gradiente da função $L(r, w)$, para obter

$$
\vec{\nabla} L(r, w)=\left(-f^{\prime}(r) / 2, w\right)
$$

Avaliando o produto escalar,

$$
\vec{z}(r, w) \cdot \vec{\nabla} L(r, w)=-w f^{\prime}(r) / 2+f^{\prime}(r) w / 2=0 \quad \therefore \quad \vec{z} \perp \vec{\nabla} L \forall(r, w),
$$

vemos que a condição de ortogonalidade entre o campo gradiente e a superfície de nível para uma função de Lyapunov é satisfeita. Agora, basta mostrar que o ponto fixo é um ponto de mínimo da função de Lyapunov $L$, para isso avaliamos a sua matriz Hessiana,

$$
\mathbf{H}_{L(r, w)}=\left(\begin{array}{cc}
-f^{\prime \prime}(r) / 2 & 0 \\
0 & 1
\end{array}\right)
$$

de onde podemos observar que o ponto fixo será um mínimo se $f^{\prime \prime}(r)<0$, formando assim um centro no espaço de estados do sistema. Em geral os centros previstos pela estabilidade linear são facilmente desfeitos por pequenas não linearidades, no entanto, os centros de sistemas conservativos são robustos (DOERING; LOPES, 2008; STROGATZ, 2018).

Prosseguindo a análise de estabilidade via método indireto de Lyapunov, temos os coeficientes associados ao ponto fixo $r_{\star}$,

$$
\nu= \pm\left.\sqrt{\frac{f^{\prime \prime}(r)}{2}}\right|_{r_{\star}} .
$$

Assim, para $f^{\prime \prime}(r)<0$, temos uma situação de estabilidade, enquanto que para $f^{\prime \prime}(r)>0$ temos uma situação de instabilidade. 


\subsection{O BURACO DE MINHOCA WH_DS QUASE EXTREMO}

$\mathrm{Na}$ aproximação de limite quase extremo, se faz interessante o cálculo de algumas quantidades associadas à geometria, tais como a gravidade superficial e o potencial efetivo associado à partícula. Avaliando a gravidade superficial no horizonte de cosmológico, obtemos a seguinte expressão,

$$
\kappa_{c}=\frac{C}{2 r_{c}} .
$$

No sistema de coordenada radial quase-global $u$ o potencial efetivo da equação diferencial (115) assume a seguinte forma,

$$
V(u)=A(r(u))\left(2 \mathscr{L}+\frac{L^{2}}{r(u)^{2}}\right) .
$$

Linearizando o potencial para geodésicas do tipo luz até termos de segunda ordem em $u$ e até a primeira ordem em $C$, temos o seguinte potencial

$$
V(u)=C\left(\frac{\Lambda L^{2}}{15}-\frac{19}{45} \Lambda^{2} L^{2} u^{2}\right)-\frac{5}{9} \Lambda^{2} L^{2} u^{2}
$$

Verifica-se que o único ponto de extremo do potencial é dado por $u=0$, pois $V(u)$ é um polinômio quadrático. Assim, o parâmetro de impacto crítico é dado pela seguinte expressão,

$$
E^{2}-V(0)=0
$$

que nos fornece diretamente como parâmetro de impacto crítico,

$$
D_{c}=\frac{L}{E}= \pm \sqrt{\frac{15}{C \Lambda}}
$$

que colocando em termos de $\kappa_{c}$, assume a seguinte forma,

$$
D_{c}= \pm \sqrt{\frac{5 r_{c}}{2 \kappa_{c}}} .
$$

Da expressão (231) podemos ver que $D_{c} \gg 1$, pois $\kappa_{c} \approx 0$. Seguindo o mesmo raciocínio, verifica-se que é suficiente considerar,

$$
\zeta=\frac{10}{r_{c}}
$$

Finalmente, o potencial ajustado com parâmetro de impacto crítico para trajetórias do tipo luz é dado por,

$$
V(u) \frac{D_{c}^{2}}{L^{2}}=-\left(\frac{25}{2 r_{c}^{3} \kappa_{c}}+\frac{19}{r_{c}^{2}}\right) u^{2}+1 .
$$


$|v|$

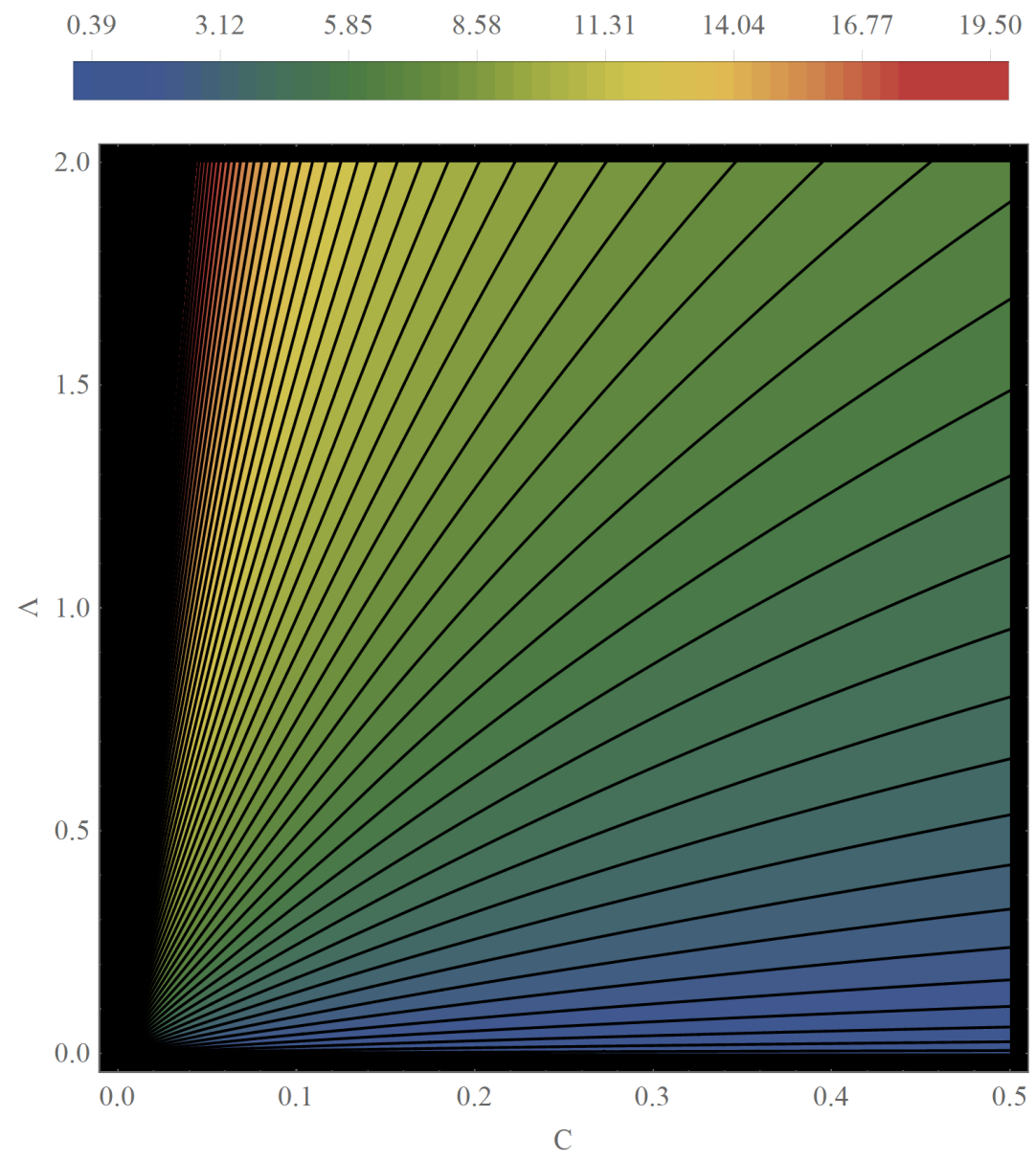

Figura 14 - Coeficientes de Lyapunov no espaço de parâmetros do sistema. Fonte: Elaborado pelo autor.

Os coeficientes de Lyapunov parametrizados pelo tempo próprio $\lambda$ são dados pela expressão (173), resultando em,

$$
\nu_{p}= \pm\left.\sqrt{\frac{-V^{\prime \prime}(u)}{2}}\right|_{u=0}= \pm \sqrt{\frac{1}{2}\left(\frac{38}{r_{c}^{2}}+\frac{25}{\kappa_{c} r_{c}^{3}}\right)}
$$

Plotando os coeficientes de Lyapunov no espaço de parâmetros, figura 14, vemos que não existe um limiar para a estabilidade, ou seja, não há nenhum tipo de bifurcação associada à troca de estabilidade de pontos fixos para as geodésicas do tipo luz críticas neste regime. Por outro lado, variando o parâmetro de impacto crítico é possível modificar topologicamente o espaço de estados do sistema, especificamente dizendo, criar ou destruir pontos fixos, conforme ilustrado na figura 15. 

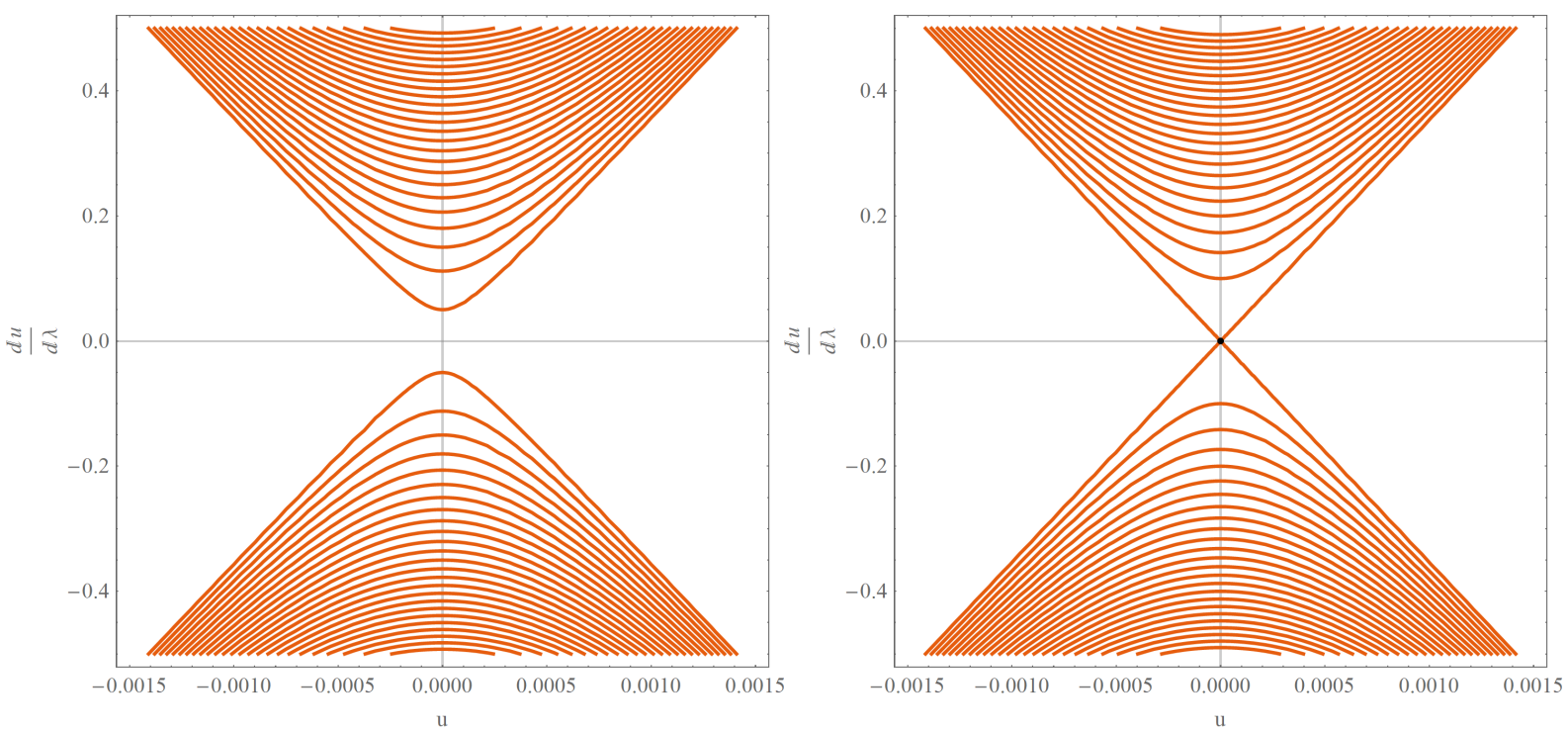

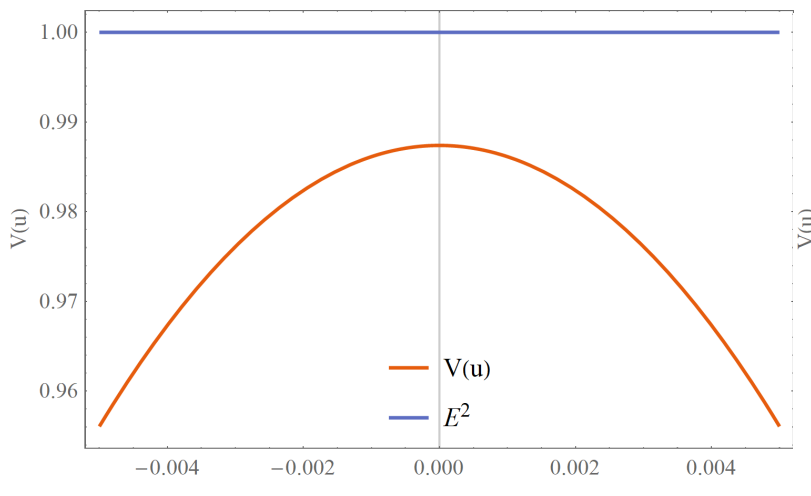

(a) Caso subcrítico $D \approx 155$.
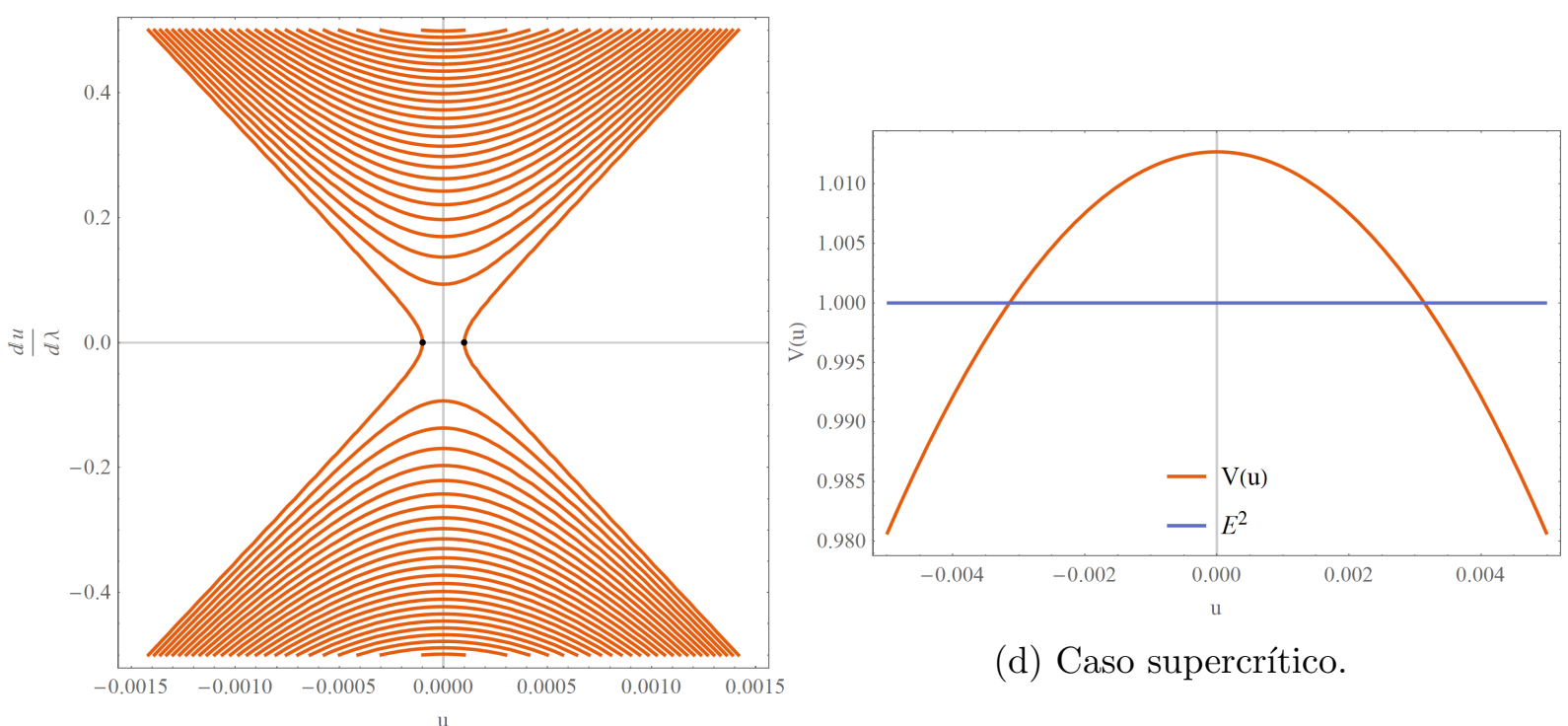

(d) Caso supercrítico.

(c) Caso supercrítico, $D \approx 161$.

Figura 15 - Bifurcação fold do sistema com o parâmetros $\Lambda=3$ e $C=2 \times 10^{-4}$. Fonte: Elaborado pelo autor. 
Quando o parâmetro de impacto está abaixo do valor crítico $\left(D<D_{c}\right)$ não há pontos fixos no sistema, quando se atinge o valor crítico $\left(D=D_{c}\right)$ o sistema adquire um ponto de equilíbrio exatamente na garganta do buraco de minhoca, observa-se que neste ponto são válidas as condições

$$
E^{2}-\left.V(u)\right|_{u=0}=-\left.\frac{\mathrm{d} V(u)}{\mathrm{d} u}\right|_{u=0}=0
$$

que são as condições necessárias para a caracterização da fotosfera (CARDOSO et al., 2009; HOD, 2011). Desta forma, a garganta do buraco de minhoca é uma fotosfera. Pelos coeficientes máximos de Lyapunov, vemos que esta fotosfera é instável, um ponto de sela no espaço de fases do sistema. Conforme se aumenta o valor do parâmetro de impacto acima do valor crítico $\left(D>D_{c}\right)$, este ponto de sela se bifurca em dois pontos fixos, um estável e outro instável. Para verificar a concordância com o critério de estabilidade de Jacobi, calcula-se diretamente os autovalores do tensor $P_{1}^{1}$ como definido na equação (63) do capítulo 2, seção 2.8. Colocando a equação diferencial na forma generalizada (35), vemos que

$$
G^{1}=\frac{1}{2} \frac{\mathrm{d}^{2} V}{\mathrm{~d} u^{2}}
$$

de onde é direto que as conexões $N_{1}^{1}=G_{11}^{1}=0$. Avaliando agora o tensor de curvatura de desvio (63) aplicado a este sistema na fotosfera, temos apenas o termo restante,

$$
P_{1}^{1}=-\left.\frac{1}{2} \frac{\mathrm{d}^{2} V}{\mathrm{~d} u^{2}}\right|_{u=0}
$$

cujo autovalor associado é

$$
\nu=P_{1}^{1}=-\left.\frac{1}{2} \frac{\mathrm{d}^{2} V}{\mathrm{~d} u^{2}}\right|_{u=0} .
$$

Segundo a definição de estabilidade 2.28, o sistema é estável no sentido de Jacobi apenas quando $V^{\prime \prime}(u)>0$. Da expressão do potencial para as órbitas críticas (233), vemos que

$$
\frac{\mathrm{d}^{2} V}{\mathrm{~d} u^{2}}=-\left(\frac{25}{r_{c}^{3} \kappa_{c}}+\frac{38}{r_{c}^{2}}\right)
$$

ou seja $V^{\prime \prime}(u)<0 \forall u \in \mathbb{R}$, caracterizando assim a instabilidade no sentido de Jacobi em todo o espaço de parâmetros. A partir da natureza do potencial, é ainda possível observar a não existência de órbitas homoclínicas e nem de órbitas heteroclínicas. 


\subsection{ANÁLISE DINÂMICA DA GEOMETRIA CFM}

Inicialmente, deseja-se descobrir os pontos fixos e eventuais pontos de equilíbrio da geometria, para isso, cancela-se o termo $A(r)$ da equação diferencial (115) e a colocamos na seguinte forma,

$$
\left(\frac{\mathrm{d} r}{\mathrm{~d} \lambda}\right)^{2}=b(r) f_{\mathrm{Sch}}(r) \equiv f_{\mathrm{CFM}}(r)
$$

com

$$
b(r) \equiv 1+\frac{(C-1) M}{2 r-3 M},
$$

$\mathrm{e}$

$$
f_{\mathrm{Sch}}(r) \equiv E^{2}-A(r)\left(2 \mathscr{L}+\frac{L^{2}}{r^{2}}\right),
$$

onde $f_{\mathrm{Sch}}(r)$ foi nomeada desta forma apenas porque aquela função assume a forma do potencial do caso de Schwarzschild. Assim, para esta função são válidas as análises realizadas para aquele caso.

Desta forma, os pontos fixos do sistema serão os $r_{\star}$ que satisfazem $b\left(r_{\star}\right)=0$, bem como aqueles $r_{\star}$ que satisfazem $f_{\text {sch }}\left(r_{\star}\right)=0$ em (240). Observa-se que a raiz do primeiro termo não depende da energia total do sistema, sendo um ponto fixo inerente da geometria, enquanto os outros pontos fixos dependem da energia total do sistema.

A raiz de $b(r)$ é dada por

$$
r_{\star}(C)=\frac{(4-C) M}{2},
$$

enquanto a raiz de $f_{\text {sch }}(r)$ será dada pelos valores de $r_{\star}$ que satisfazem a equação algébrica,

$$
E^{2}=A\left(r_{\star}\right)\left(2 \mathscr{L}-\frac{L^{2}}{r_{\star}^{2}}\right) .
$$

Observa-se da equação (243) que o ponto fixo oriundo do termo $b(r)$ só se torna relevante para valores de $C \in \mathbb{R}^{-}$, uma vez que para valores de $C>0$ o ponto fixo originado por esta função está contido dentro do horizonte de eventos em $r=2 M$, região esta que não é mapeada pela carta $\{t, r, \theta, \phi\}$. Tal comportamento pode ser visto pela figura 16 .

Primeiramente, estudemos um tipo simples de órbitas, as radiais. Para tal é possível empregar o métodos das isóclinas, como apresentadas na definição 2.3. As órbitas radiais 


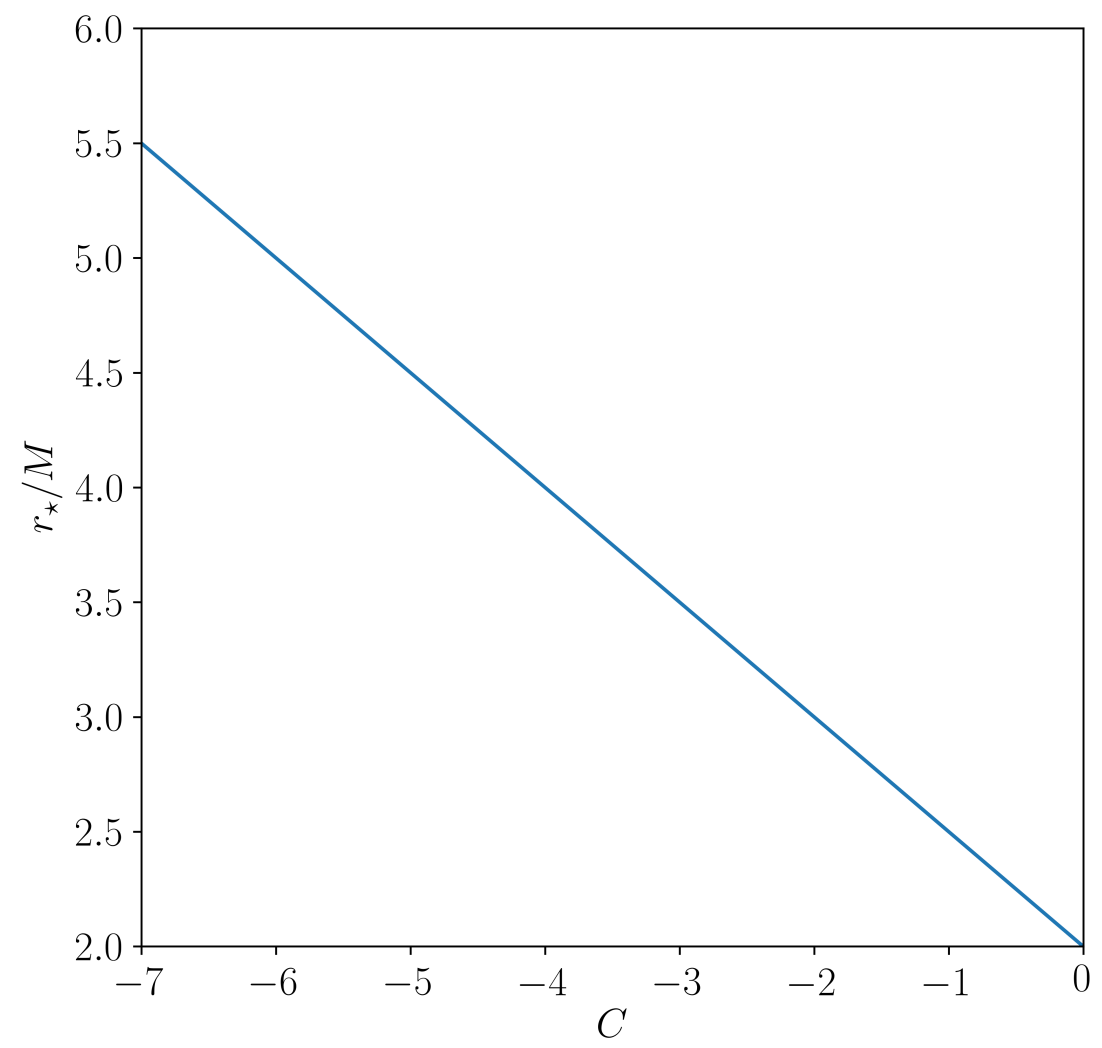

Figura 16 - Gráfico do raio da garganta do buraco de minhoca em função do parâmetro $C$.

Fonte: Elaborado pelo autor.

possuem momento angular nulo, o que segundo as equações (117), (190) e (191) faz com que as equações de movimento assumam a seguinte forma ,

$$
\begin{aligned}
& \frac{\mathrm{d} x}{\mathrm{~d} \lambda}=\frac{\mathrm{d} r}{\mathrm{~d} \lambda} \frac{x(\lambda)}{\sqrt{x(\lambda)^{2}+y(\lambda)^{2}}}, \\
& \frac{\mathrm{d} y}{\mathrm{~d} \lambda}=\frac{\mathrm{d} r}{\mathrm{~d} \lambda} \frac{y(\lambda)}{\sqrt{x(\lambda)^{2}+y(\lambda)^{2}}} .
\end{aligned}
$$

Segundo a definição 2.3, vemos que as isóclinas do sistema devem obedecer a equação,

$$
\frac{\mathrm{d} y}{\mathrm{~d} x}=\frac{y}{x}=k
$$

ou seja as isóclinas de inclinação $k$ são as retas de equação,

$$
y=k x
$$

Os espaços de estados no plano orbital das trajetórias radiais estão diagramados na figura 17. Observa-se que para este caso, não existem órbitas fechadas e, consequentemente, não existem ciclos, apenas trajetórias puramente radiais. 


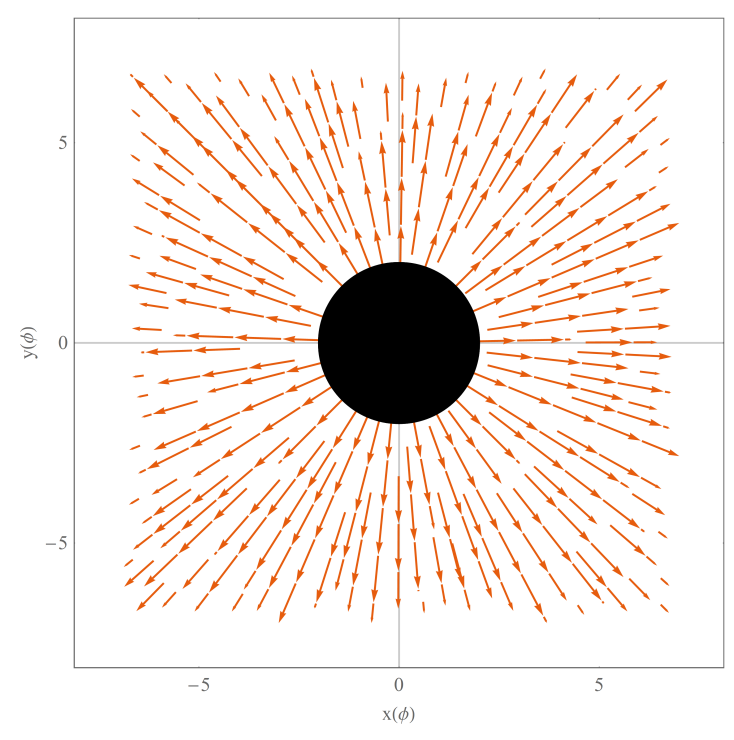

(a) Órbitas radiais com $\dot{r}>0$.

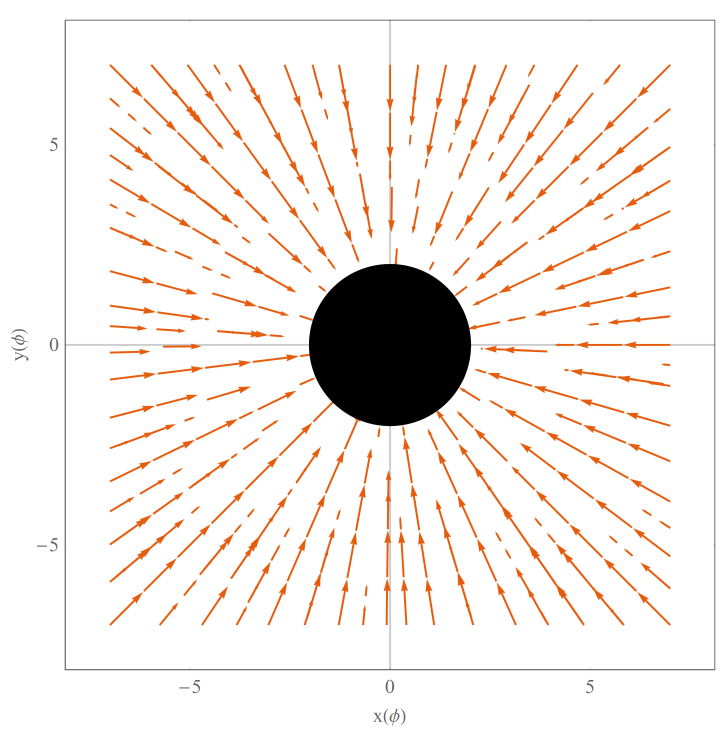

(b) Órbitas radiais com $\dot{r}<0$.

Figura 17 - Órbitas radiais se aproximando e se afastando do buraco negro. A região em preto é a região do horizonte de eventos em $r=2 M$.

Fonte: Elaborado pelo autor.

\subsection{FOTOSFERAS CFM}

A fotosfera é a única região que permite órbitas circulares no sistema, em outras palavras, órbitas cujo o raio é invariante mediante a evolução temporal do sistema. Segundo a condição apresentada em (CARDOSO et al., 2009; HOD, 2011), as órbitas circulares devem satisfazer a seguinte condição,

$$
f_{\mathrm{CFM}}\left(r_{c}\right)=f_{\mathrm{CFM}}^{\prime}\left(r_{c}\right)=0
$$

Colocando a equação de movimento no plano orbital ao considerar $r$ como uma função de $\phi$ ao invés de $\lambda$, e ainda realizando uma mudança de variáveis $u=r^{-1}$ obtemos

$$
\left(\frac{\mathrm{d} u}{\mathrm{~d} \phi}\right)^{2}=\left[1+\frac{(C-1) M u}{2-3 M u}\right]\left[2 M u^{3}-u^{2}+\frac{4 M \mathscr{L} u}{L^{2}}-\frac{2 \mathscr{L}-E^{2}}{L^{2}}\right] .
$$

Para buscar a fotosfera, devemos utilizar a condição para geodésicas nulas $\mathscr{L}=0$, isso nos fornece,

$$
\left(\frac{\mathrm{d} u}{\mathrm{~d} \phi}\right)^{2}=\left[1+\frac{(C-1) M u}{2-3 M u}\right] \overbrace{\left[2 M u^{3}-u^{2}+\frac{1}{D^{2}}\right]}^{f_{\mathrm{sch}}(u)} .
$$

$\operatorname{com} D=L / E$. 
Da análise em Schwarzschild, sabemos que como $f_{\text {sch }}(u)$ é polinomial de grau três em $u$, então existem três raízes complexas moduladas pelo termo independente $D^{2}$. Derivando o polinômio em relação à $u$, temos,

$$
\frac{\mathrm{d} f_{\mathrm{sch}}}{\mathrm{d} u}=6 M u^{2}-2 u,
$$

cuja raiz positiva é

$$
u_{1}=\frac{1}{3 M}
$$

O valor de $f_{\text {CFm }}$ neste ponto é,

$$
f_{\text {CFM }}\left(3 M^{-1}\right)=[(C-1) M+1]\left(\frac{E^{2}}{L^{2}}-\frac{1}{27 M^{2}}\right),
$$

ou seja, o ponto $u=3 M^{-1}$ será uma raiz de multiplicidade algébrica dupla quando $C_{\mathrm{c}}=(M-1) / M$ ou $D_{\mathrm{c}}=3 \sqrt{3} M$. Estes dois parâmetros serão importantes pois eles são geradores de bifurcação no sistema, como veremos mais adiante. Em suma, as soluções do sistema (249) são,

$$
\begin{gathered}
D_{1}= \pm 3 \sqrt{3} M \quad \text { com } \quad r_{1}=3 M, \\
D_{2}= \pm \frac{(C-4)^{\frac{3}{2}} M}{2 \sqrt{C}} \quad \text { com } \quad r_{2}=\frac{M}{2}(4-C),
\end{gathered}
$$

ou seja há duas fotosferas no sistema para $C \neq-2$.

A dinâmica para os casos de buracos negros com singularidade interna $(C>1)$ segue a dinâmica de Schwarzschild, já descrita na seção 4.2. A análise qualitativa do sistema é levemente diferente para o caso de buraco negro sem singularidade interna $(C \in] 0,1[)$, como deve ser observado no retrato de fases ilustrado na figura 18.

Observa-se que além do ponto de sela tradicionalmente associado à fotosfera em $r=3 M$ existe também um centro entre a fotosfera e o horizonte de eventos em $r=2 M$. Este centro é inacessível para $C>0$ uma vez que o parâmetro de impacto necessário é complexo. Observa-se também que uma órbita homoclínica é gerada nesta região e, eventualmente, destruída com a varição dos parâmetros do sistema.

\subsection{BIFURCAÇÕES DO SISTEMA E ÓRBITA HOMOCLÍNICA}

Tomando a equação (240) como um sistema unidimensional, observa-se que a codimensão de bifurcação, ou seja, o número de parâmetros que devem ser variados a fim 


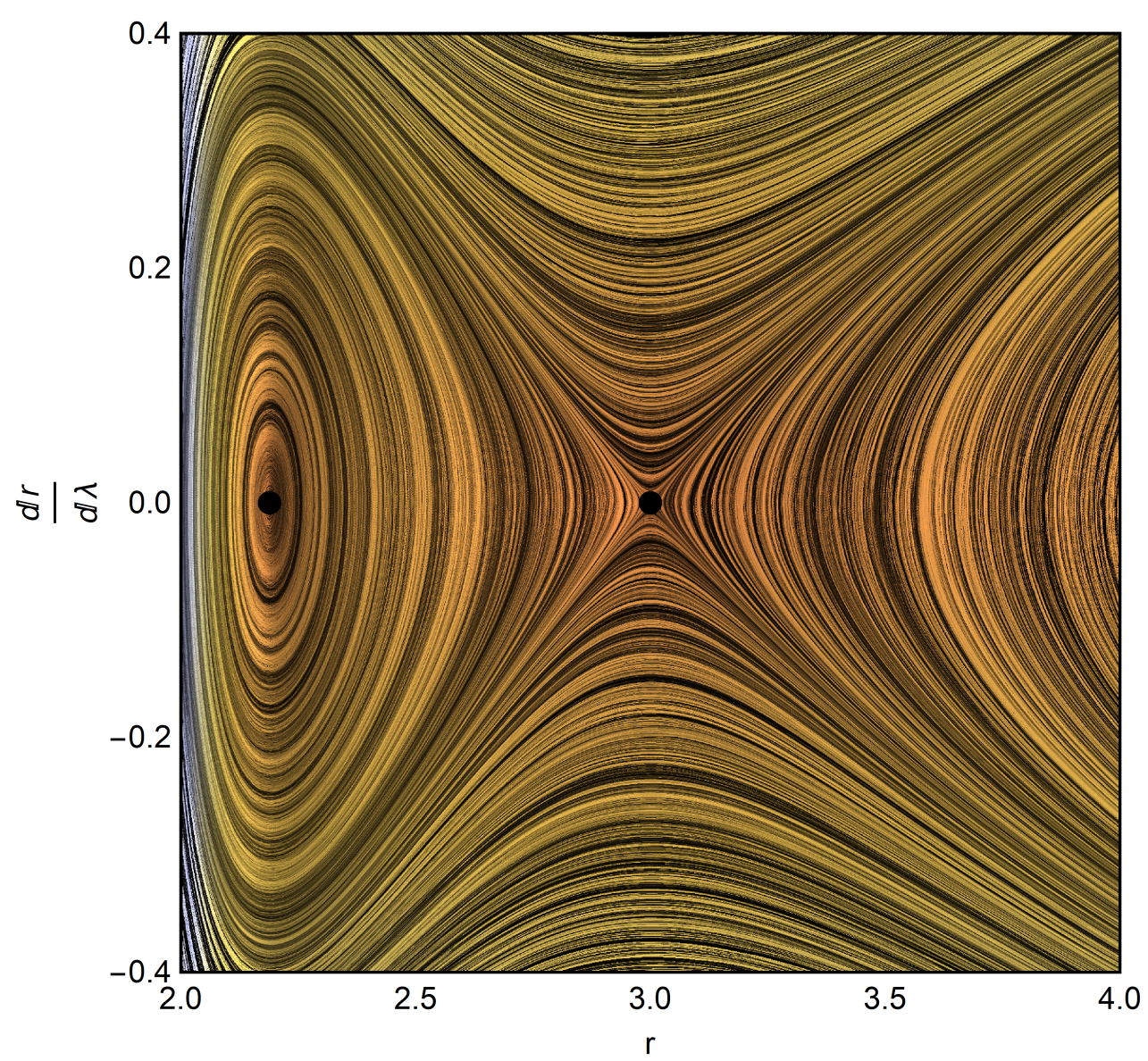

Figura 18 - Retrato de fase do sistema com $C=0.01, M=1, D_{c}=3 \sqrt{3} M$ e $E=1$. Fonte: Elaborado pelo autor.

de produzir a bifurcação (STROGATZ, 2018), é dois. Especificamente, os parâmetros do sistema que geram a bifurcação são $D$ e $C$.

Variando o parâmetro de impacto podemos criar e destruir pontos fixos do sistema, bem como gerar ou destruir a órbitas que tendem à fotosfera, observe as figuras 19. A imagem 19a mostra os pontos fixos do sistema para valores do parâmetro de impacto acima do valor crítico, enquanto a figura 19b mostra o sistema no valor de bifurcação do parâmetro de impacto crítico, quando os dois pontos fixos se fundem em um, originando assim um ciclo limite. A figura 19c mostra os pontos fixos do sistema com o parâmetro de impacto abaixo do valor cítico de bifurcação. 


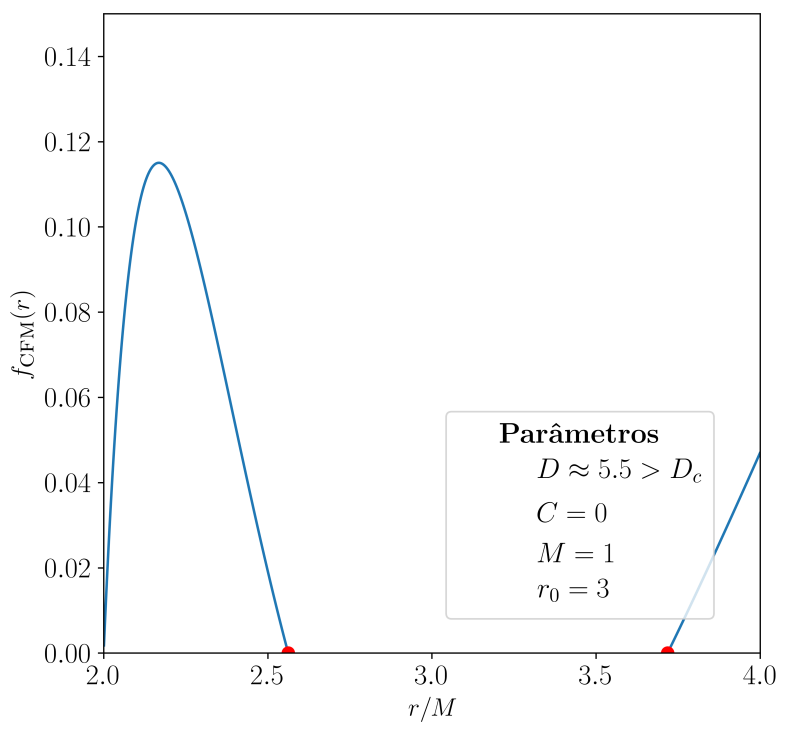

(a)

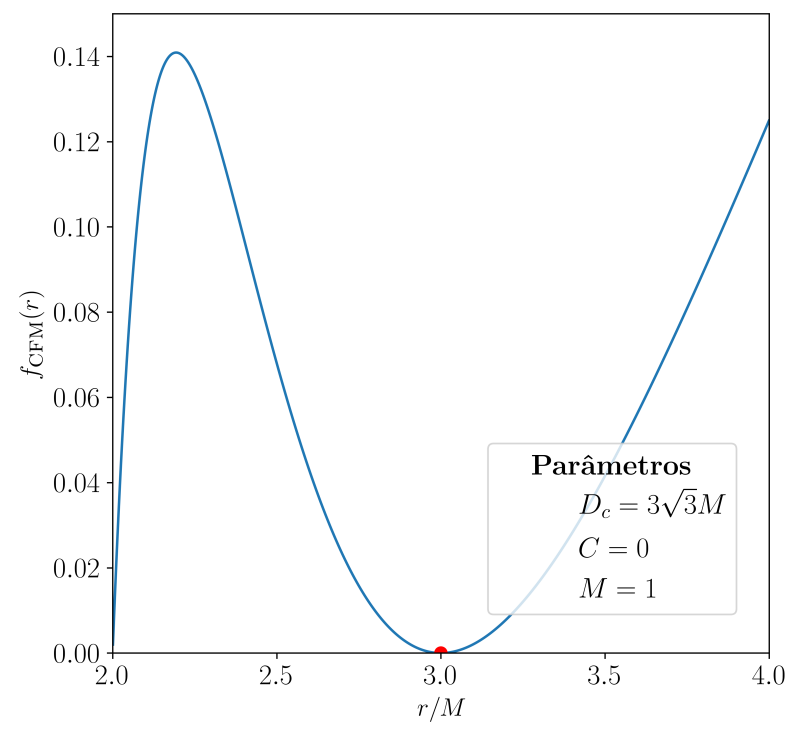

(b)

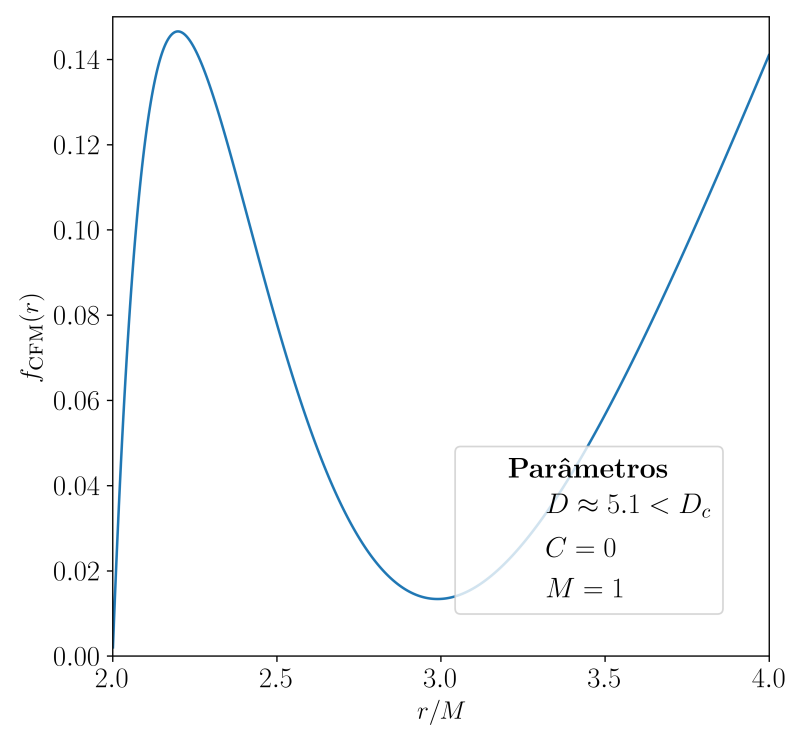

(c)

Figura 19 - Função $f_{\text {CFM }}(r)$ plotada para alguns valores de parâmetro de impacto $D$. Fonte: Elaborado pelo autor.

As trajetórias associadas a cada uma das situações da figura 19 estão na figura 20. 


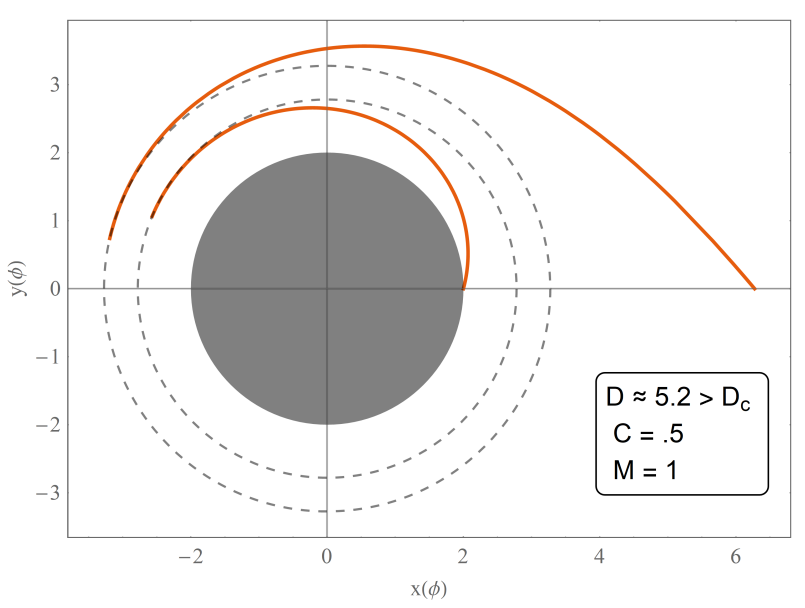

(a)

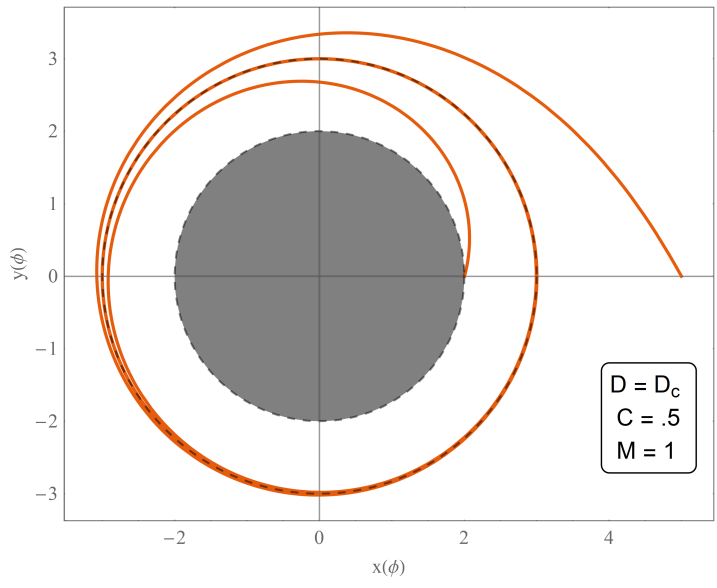

(b)

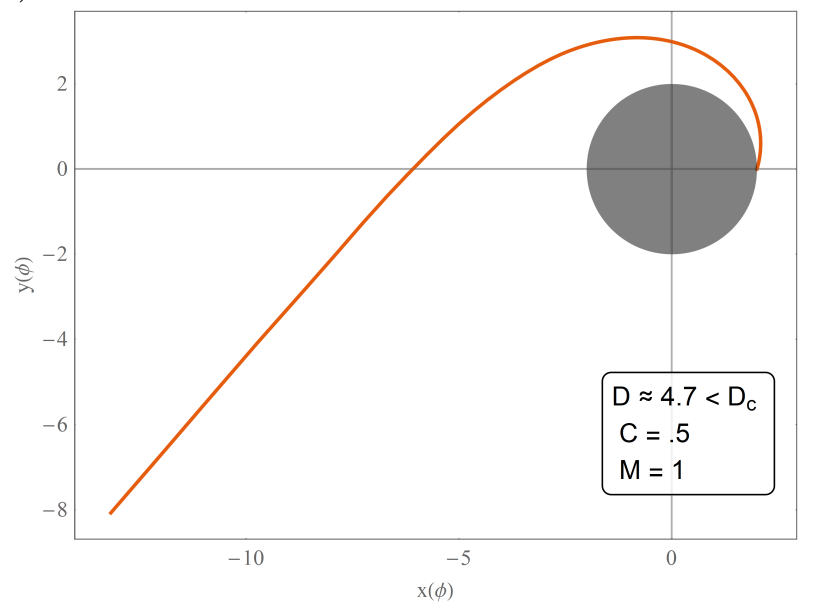

(c)

Figura 20 - Possíveis casos a partir do parâmetro de bifurcação: (a) Parâmetro de impacto acima do valor crítico, (b) Parâmetro de impacto no valor crítico e (c) Parâmetro de impacto abaixo do valor crítico.

Fonte: Elaborado pelo autor.

Pode-se observar do plano orbital que quando o parâmetro de bifurcação está abaixo do valor crítico não há fotosfera e as geodésicas com $\dot{r}<0$ tendem para $r=2 M$ e as com $\dot{r}>0$ se afastam do horizonte de eventos. Esse comportamento pode ser inferido a partir da expressão (240). Observa-se também que quando estamos na bifurcação obtemos um ciclo limite semi-estável no plano orbital 20b, e ao passar deste valor obtemos dois ciclos onde a velocidade é nula, região onde ocorre a inversão da velocidade radial, isto é, a velocidade radial muda de sinal 20a. Este comportamento é característico das bifurcações do tipo fold em ciclos (STROGATZ, 2018; KUZNETSOV, 2013).

Para compreender a dinâmica de uma forma um pouco mais precisa vamos estudar os autovalores no plano orbital. Colocando o sistema em uma base cartesiana temos

$$
\dot{x}=\frac{\mathrm{d} \phi}{\mathrm{d} \lambda}\left[\frac{\mathrm{d} r}{\mathrm{~d} \phi} \frac{x}{r}-y\right],
$$




$$
\dot{y}=\frac{\mathrm{d} \phi}{\mathrm{d} \lambda}\left[\frac{\mathrm{d} r}{\mathrm{~d} \phi} \frac{y}{r}+x\right],
$$

cuja a Jacobiana associada é dada por

$$
\mathbf{J}=\left(\begin{array}{cc}
\frac{1}{r} \partial_{\lambda} r & -\partial_{\lambda} \phi \\
\partial_{\lambda} \phi & \frac{1}{r} \partial_{\lambda} r
\end{array}\right)
$$

A equação característica para os autovalores obedece a seguinte relação,

$$
\nu_{1,2}=\frac{\operatorname{tr}(\mathbf{J}) \pm \sqrt{(\operatorname{tr}(\mathbf{J}))^{2}-4 \operatorname{det}(\mathbf{J})}}{2}=\frac{1}{r} \frac{\mathrm{d} r}{\mathrm{~d} \lambda} \pm i\left|\frac{\mathrm{d} \phi}{\mathrm{d} \lambda}\right|
$$

Quando avaliado em pontos fixos, obtemos,

$$
\nu_{1,2}= \pm i\left|\frac{\mathrm{d} \phi}{\mathrm{d} \lambda}\right|
$$

que é exatamente a velocidade angular parametrizada por $\lambda$. Portanto, é uma órbita circular uma vez que a velocidade radial é nula. Em outras palavras, em qualquer outro ponto do sistema que não seja um ponto de equilíbrio temos que $\operatorname{tr}(\mathbf{J}) \neq 0$, pois a geodésica terá componente radial da velocidade não nula, caracterizando trajetórias espiralantes. A unicidade das soluções garante que não haja intersecção de duas soluções com condições iniciais diferentes no plano uma vez que o sistema de equações diferenciais ordinárias (256)-(257) é autônomo e Lipschitz contínuo em $x$ e $y$ na fotosfera. Assim, as trajetórias vizinhas às fotosferas tendem assintoticamente a elas.

Olhando o espaço de estados no plano orbital, observa-se a formação de trajetórias que tendem assintoticamente ao ciclo por uma região e trajetórias que se afastam do ciclo por outra região. Exemplos destes campos estão plotados na figura 21. Esse comportamento caracteriza um ciclo limite semi estável segundo a definição 2.14.

O fato deste ciclo limite ser semi-estável pode ser inferido do espaço de estados do sistema, uma vez que a fotosfera é um ponto de sela neste espaço e existe uma variedade estável e outra instável associada a este ponto, o que se reflete no espaço de estados do plano orbital. 


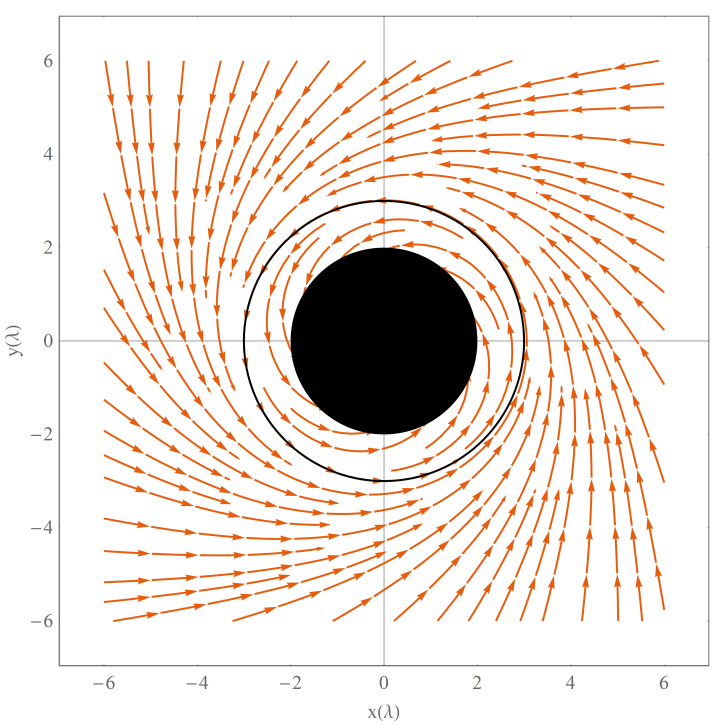

(a) $L>0$ e $\dot{r}<0$.

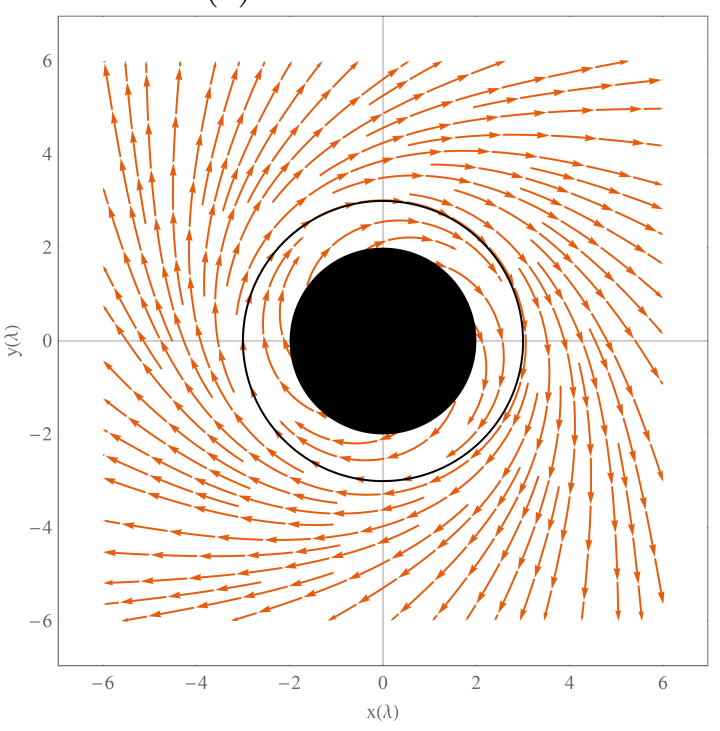

(c) $L<0$ e $\dot{r}>0$.

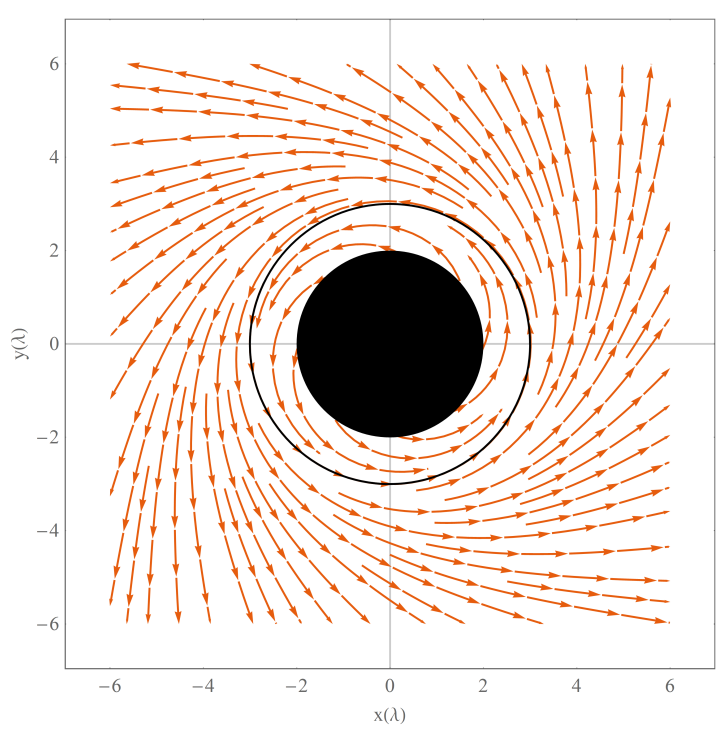

(b) $L>0$ e $\dot{r}>0$.

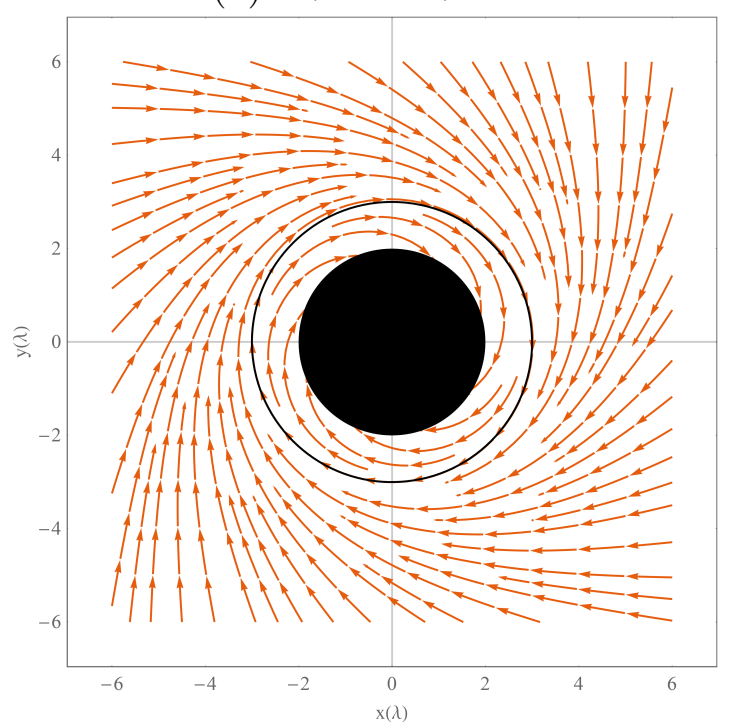

(d) $L<0$ e $\dot{r}<0$.

Figura 21 - Espaços de estados no plano orbital para diversos valores de $L$ e $\dot{r}$. A circunferência de preto representa a fotosfera em $r=3 M$ enquanto o disco negro é o horizonte de eventos em $r=2 M$.

Fonte: Elaborado pelo autor.

As figuras 21a e 21b representam trajetórias que se iniciam no infinito e tendem assintoticamente ao ciclo, bem como as trajetórias que se iniciam na proximidade do ciclo e caem no horizonte de eventos. Por outro lado, as figuras 21c e 21d apresentam trajetórias que se iniciam na periferia do ciclo e tendem ao infinito conforme a evolução do sistema, bem como trajetórias que se iniciam próximas ao horizonte de eventos e tendem ao ciclo limite conforme o sistema evolui $\lambda \rightarrow \infty$.

Conforme colocado nas definições 2.9 e 2.10, a região de atração associada aos espaços 21 a e 21d é toda a região compreendida por $r>3 M$, enquanto a região de repulsão 
é a região compreendida por $2 M<r<3 M$, por outro lado, a região de atração associada às figuras $21 \mathrm{~b}$ e $21 \mathrm{c}$ é a região $2 M<r<3 M$ e a de repulsão $r>3 M$.

Por outro lado, ao variar o parâmetro $C$ no sistema, é possível observar a aniquilação de pontos fixos e troca de estabilidade do mesmos. Tomemos a situação onde $C<0$ e vejamos o comportamento do sistema com a variação do parâmetro. Para esse regime o valor crítico do parâmetro é dado por $C_{c}=-2$, que é exatamente o limiar onde os dois pontos fixos do sistema (de centro e de sela) se fundem em um único ponto fixo do tipo cúspide, invertendo a estabilidade conforme diminui $C$. A dinâmica é explicitada na figura 22 onde são plotados os espaços de estado para diversos valores de $C$.

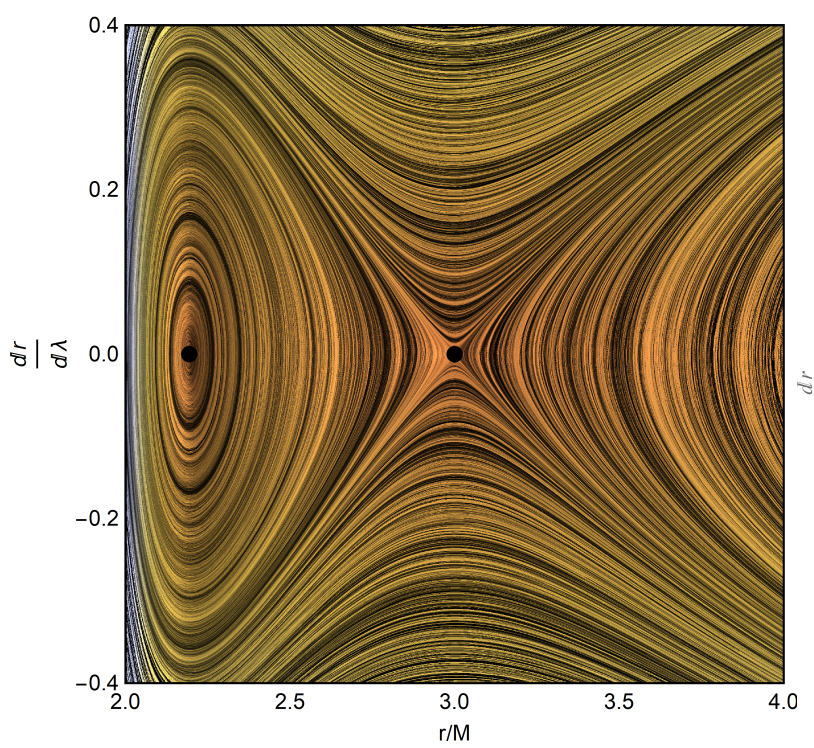

(a)

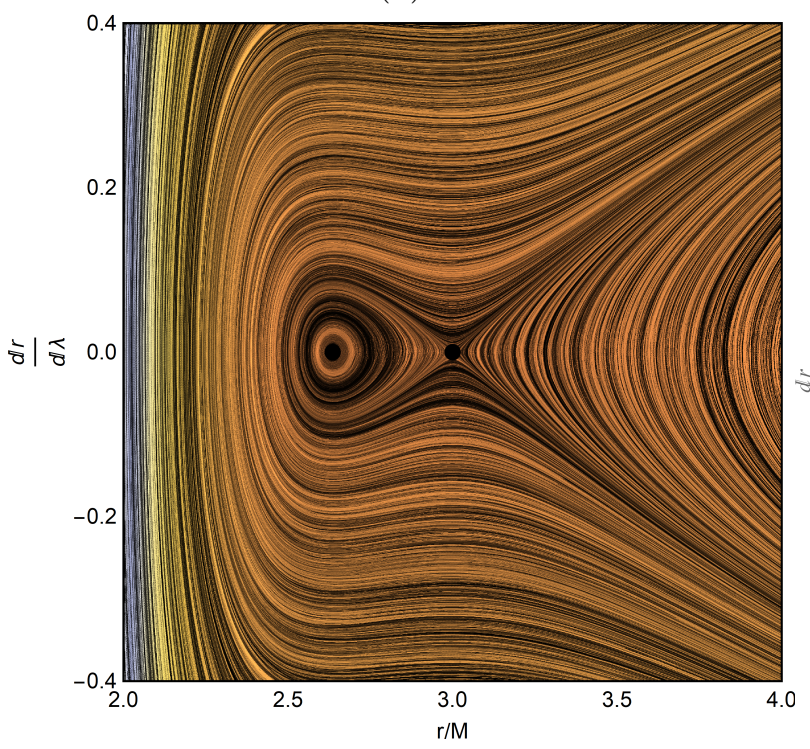

(b)
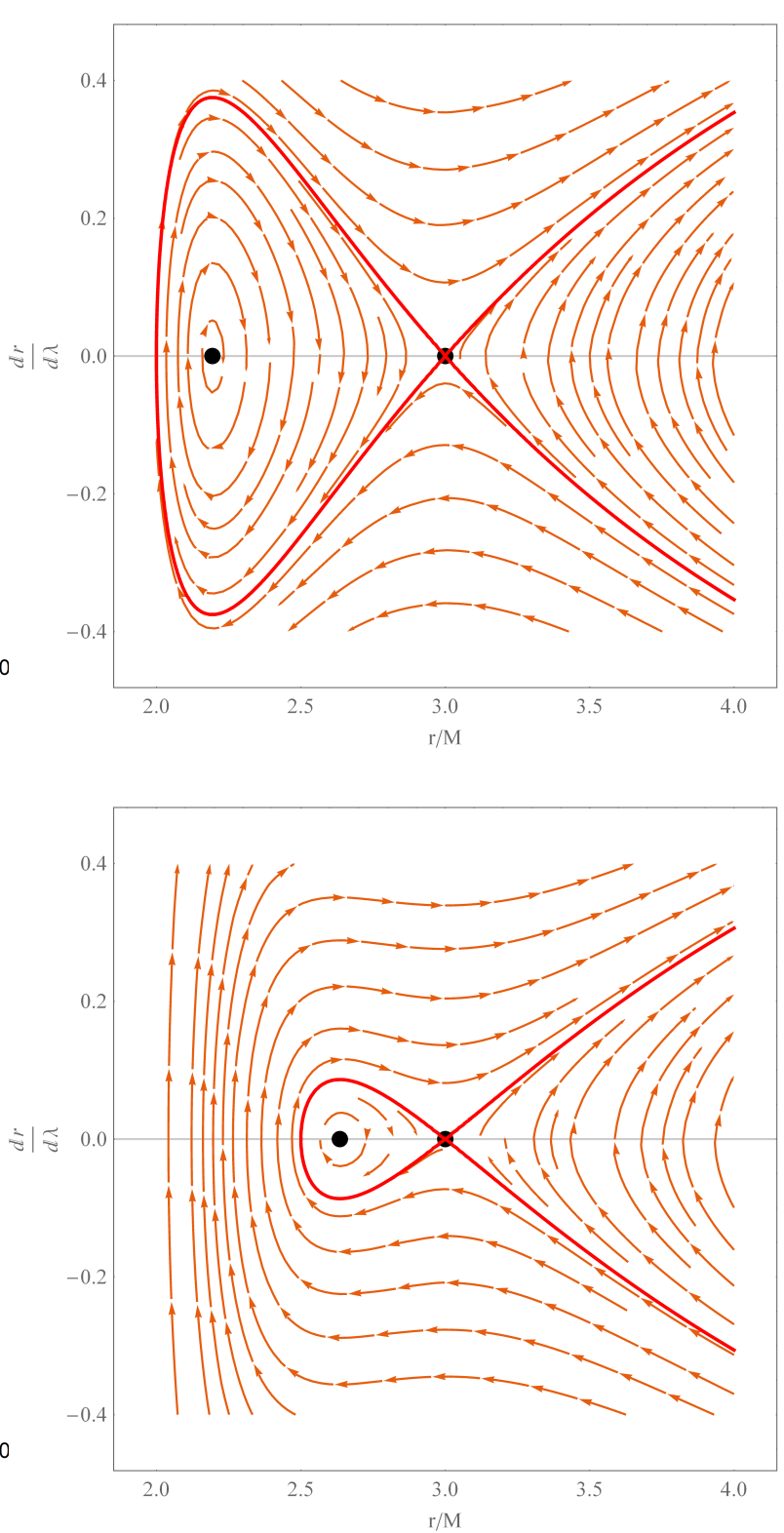


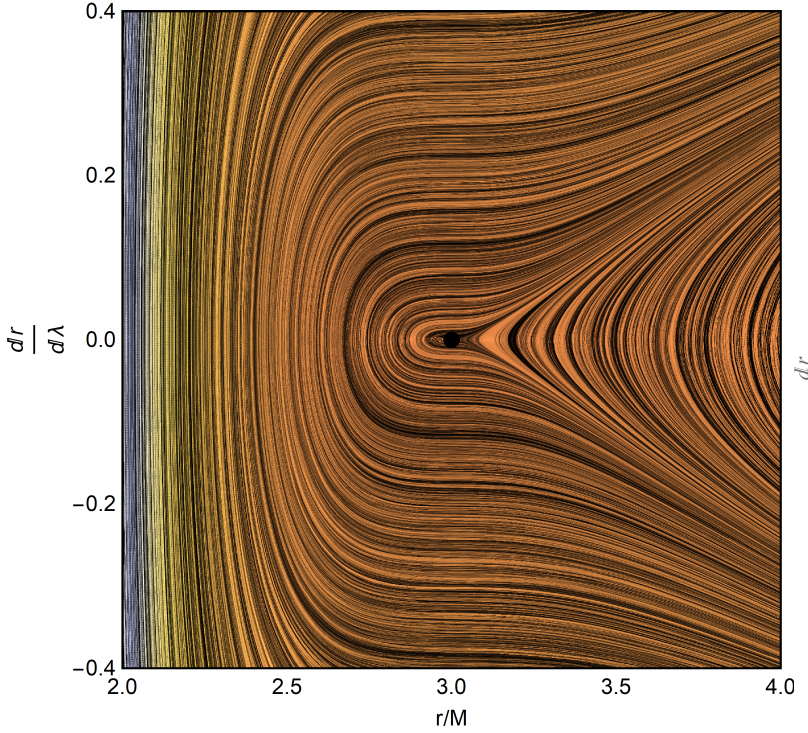

(c)

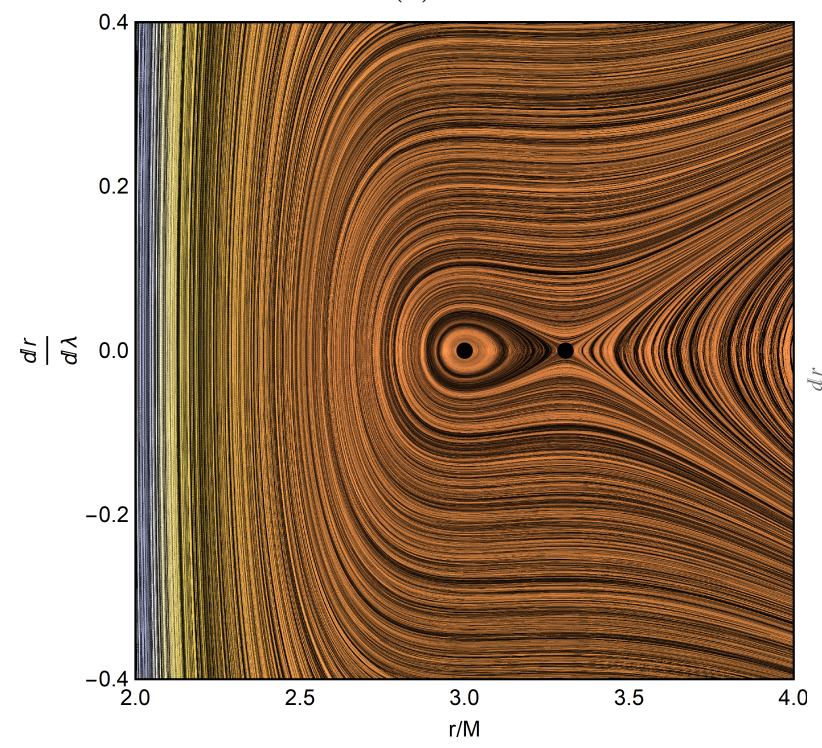

(d)
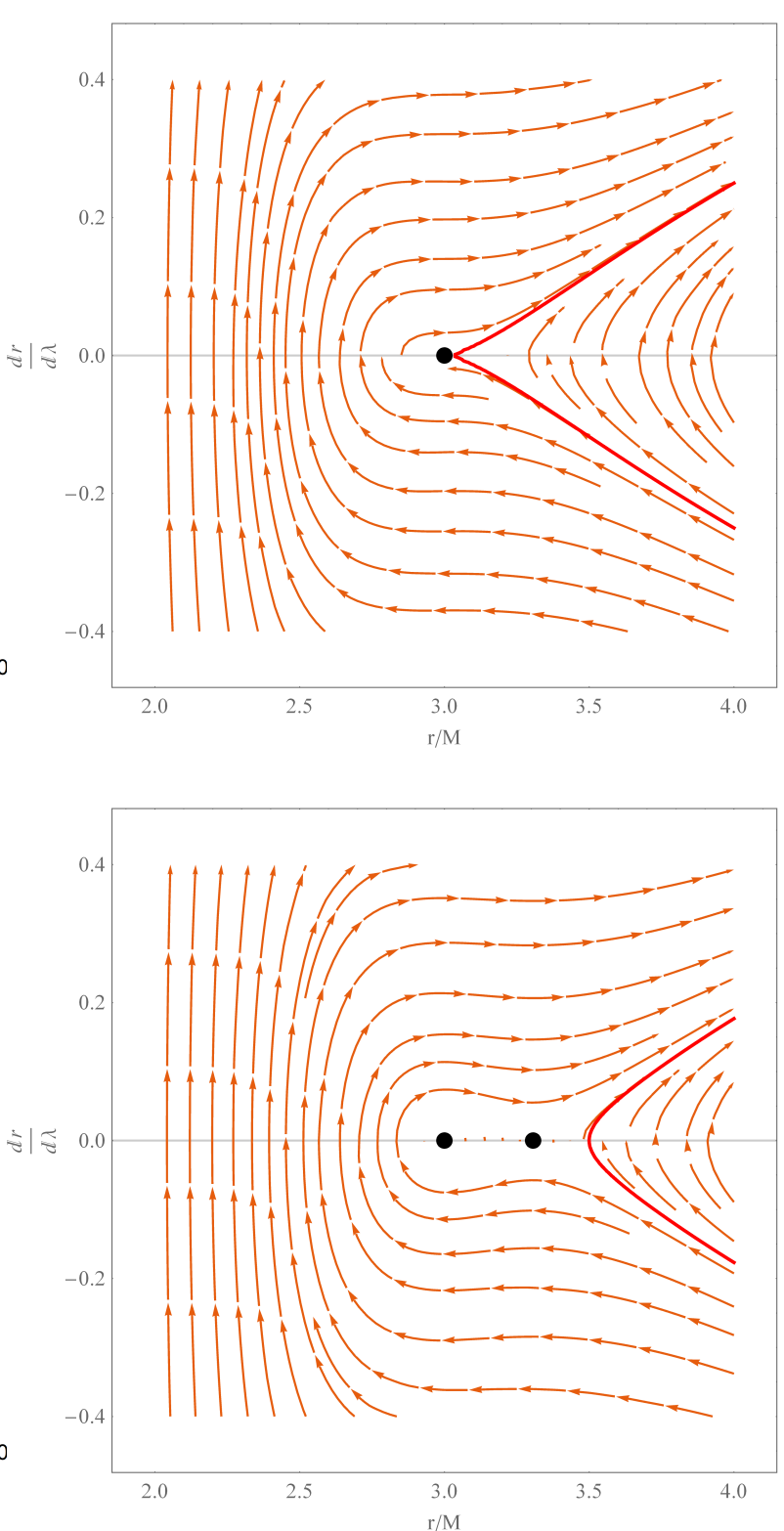

Figura 22 - Possíveis casos a partir do parâmetro de bifurcação $C$ : (a) Caso supercrítico $C=0$, (b) Caso supercrítico $C=-1$ (c) Caso crítico $C_{c}=-2$ e (d) Caso subcrítico $C=-3<C_{c}$.

Fonte: Elaborado pelo autor.

Conforme podemos observar nos retratos de fase do sistema, vemos que a sua topologia se altera conforme ocorre a colisão do centro com a sela, esta bifurcação é muito comum em sistemas conservativos, se chama bifurcação centro-sela (HALE; KOÇAK, 2012). 
Além da bifurcação é possível observar a existência de uma órbita homoclínica nos casos onde $-2<C<0$, representada em vermelho na coluna da direita em 22a e 22b. Esquematicamente, é possível plotar a variedade de equilíbrio do sistema definida por,

$$
f_{\mathrm{CFM}}\left(r_{\star}\right)=0,
$$

como uma função de $D$ ou $C$. Os gráficos resultantes são apresentados nas figuras 23a e $23 \mathrm{~b}$.

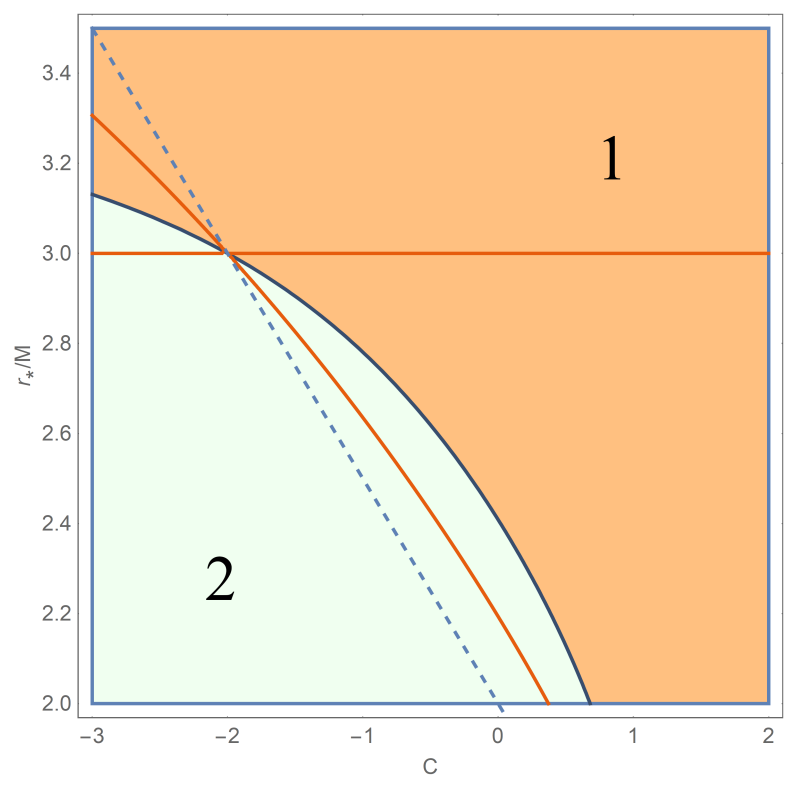

(a)

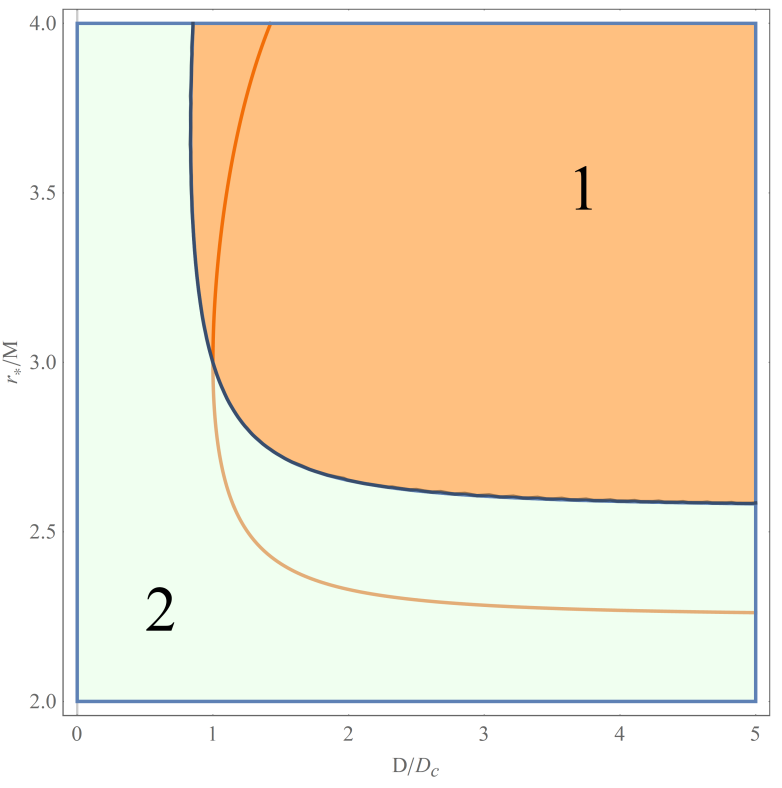

(b)

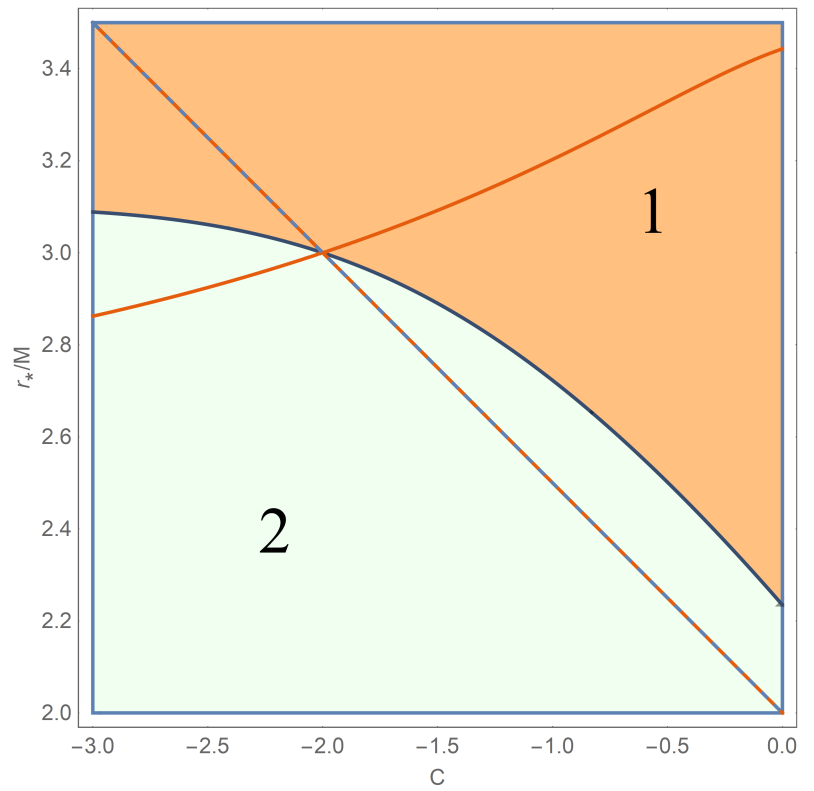

(c)

Figura 23 - (a) Variedade de equilíbrio em função de $C$ com $D=3 \sqrt{3} M$, (b) variedade de equilíbrio do sistema em função de $D$ e (c) Variedade de equilíbrio em função de $C$ com $D=(C-4)^{3 / 2} M / 2 C^{1 / 2}$. 
Na figura 23, as regiões em laranja (região 1) indicam zonas de instabilidade enquanto as regiões em verde (região 2) indicam zonas de estabilidade do sistema, enquanto a curva tracejada é a variedade do raio da garganta. No diagrama acima ficam evidentes as bifurcações com relação à quantidade de pontos fixos, bem como em relação às suas estabilidades. Estas bifurcações menores (de codimensão 1) geram uma bifurcação Bogdanov-Takens. Observa-se que quando o sistema está com o parâmetro crítico de impacto $D_{c}$ e no valor $C_{c}$, ambas as fotosferas coincidem, gerando um ponto fixo degenerado. Tal fato nos indica que uma bifurcação de codimensão maior está ocorrendo, uma bifurcação de codimensão 2 .

A equação para o movimento geodésico na geometria CFM pode ser colocada na seguinte forma,

$$
\frac{\mathrm{d}^{2} r}{\mathrm{~d} \lambda^{2}}=\frac{1}{2} \frac{\mathrm{d} f}{\mathrm{~d} r}
$$

que por sua vez ainda pode ser desacoplada no seguinte sistema de equações diferenciais ordinárias lineares,

$$
\begin{aligned}
& \dot{r}=w, \\
& \dot{w}=\frac{1}{2} f_{\mathrm{CFM}}^{\prime}(r),
\end{aligned}
$$

com

$$
f_{\mathrm{CFM}}(r)=\left[1+\frac{(C-1) M}{2 r+3 M}\right]\left[1+A(r)\left(\mathscr{L}+\frac{D^{2}}{r^{2}}\right)\right],
$$

onde os parâmetros $C$ e $D$ são os parâmetros livres.

Para rastrear tal bifurcação, avaliemos a matriz de estabilidade linear do sistema $(263)$,

$$
\mathbf{J}=\left(\begin{array}{cc}
0 & 1 \\
\frac{1}{2} f_{\mathrm{CFM}}^{\prime \prime}(r) & 0
\end{array}\right)_{\left(r_{*}, C_{*}, D_{*}\right)}
$$

Realizando as seguintes mudanças de coordenadas e parâmetros,

$$
\begin{aligned}
& \tilde{r}=r-3 M, \\
& \tilde{C}=C+2, \\
& \tilde{D}=D-3 \sqrt{3} M,
\end{aligned}
$$

fazemos com que o valores de bifurcação sejam $(\tilde{r}, \tilde{C}, \tilde{D})=(0,0,0)$. Avaliando a matriz (265) nos valores de bifurcação, temos

$$
\mathbf{J}=\left(\begin{array}{ll}
0 & 1 \\
0 & 0
\end{array}\right)
$$


cujos autovalores são $\nu_{1,2}=0$, indicando assim pela definição 2.34 a ocorrência assim de uma BBT no sistema. O próximo passo é o cálculo dos coeficientes da forma normal desta bifurcação, que podem ser encontrados no apêndice A. Os coeficientes assumem os seguintes valores,

$$
\begin{aligned}
& a_{2}=\frac{1}{3}, \\
& a_{3}=-\frac{56}{81}, \\
& b_{2}=b_{3}=a_{4}=b_{4}=0,
\end{aligned}
$$

o que mostra que o nosso sistema de fato passa por uma BBT-degenerada com $b_{2}=0$ e $a_{2} \neq 0$, sendo então localmente topologicamente equivalente ao sistema,

$$
\begin{aligned}
& \dot{\xi}_{0}=\xi_{1} \\
& \dot{\xi}_{1}=\beta_{1}+\beta_{2} \xi_{0}+\frac{1}{3} \xi_{0}^{2},
\end{aligned}
$$

onde a BBT de codimensão dois ocorre.

Comparando a dinâmica do sistema (269) com a dinâmica do sistema (263) observamos que o parâmetro $\beta_{1}$ desempenha o papel do parâmetro de impacto $D$ e o parâmetro $\beta_{2}$ desempenha o papel de $C$. Então, para compreender o comportamento da bifurcação é suficiente analisar o sistema (269). Ainda mais, localmente (entenda-se localmente como no limite onde os parâmetros estão arbitrariamente próximo dos valores de bifurcação) o sistema apresenta a topologia equivalente à,

$$
\begin{aligned}
& \dot{\xi}_{0}=\xi_{1}, \\
& \dot{\xi}_{1}=\frac{1}{3} \xi_{0}^{2},
\end{aligned}
$$

ou seja, um ponto de cúspide instável, figura 24. O sistema (269) apresenta dois pontos fixos,

$$
\left(r_{\star}, w_{\star}\right)_{1,2}=\left(\frac{3}{2} \frac{\sqrt{3 \tilde{C}^{2}-4 \tilde{D}}}{\sqrt{3}} \pm \frac{3}{2} \tilde{C}, 0\right),
$$

que coalescem na origem quando $\tilde{D}=\tilde{C}=0$, formando a cúspide. 

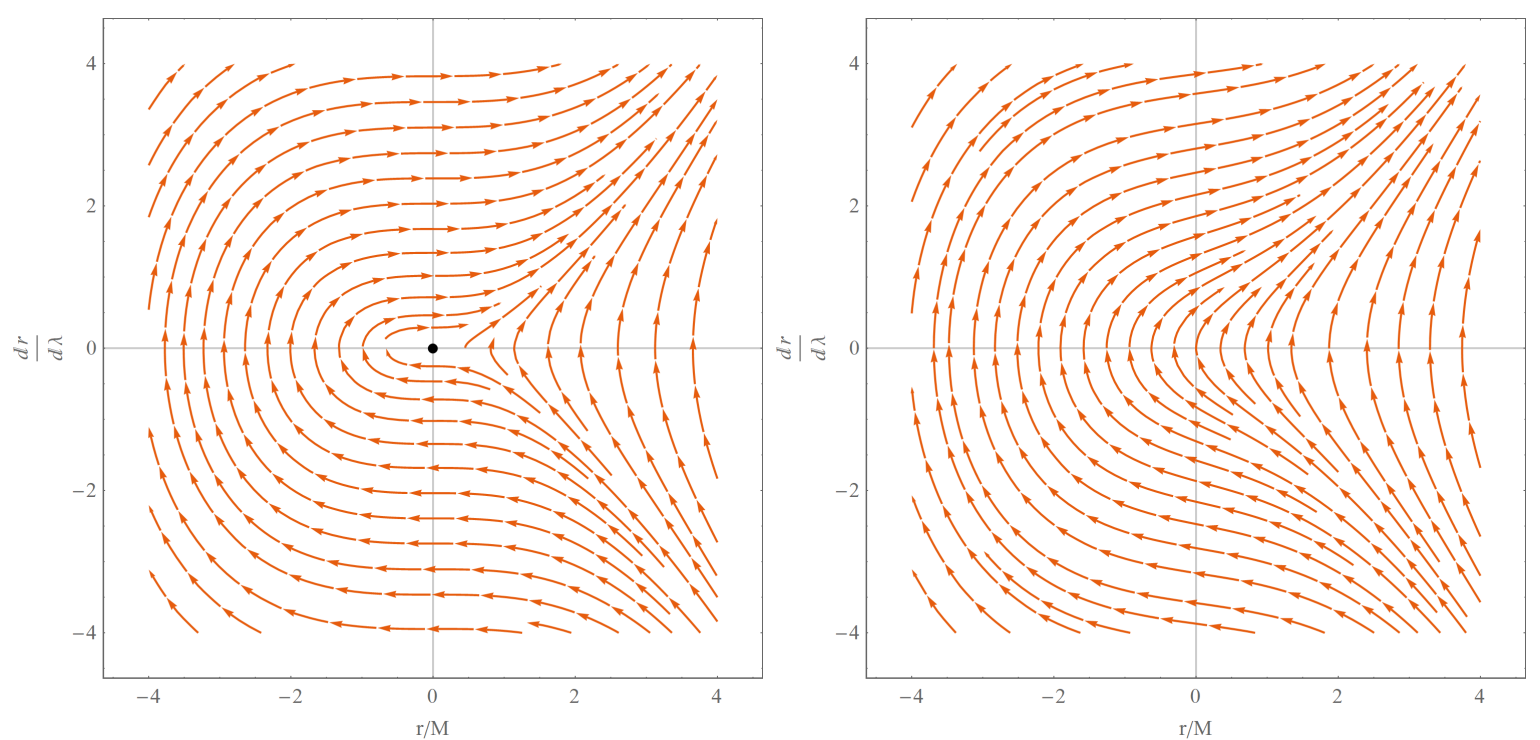

Figura 24 - A esquerda, espaço de estados do sistema (270), a direita, espaço de estado do sistema (269) após a ocorrência da fold.

Fonte: Elaborado pelo autor.

Verifica-se uma fold ocorrendo em $D_{c}$ aniquilando os pontos fixos, gerando o o gráfico à direta de da figura 24. Como este caso de BBT é degenerado, a forma normal da bifurcação é dada por (270). Observa-se a partir dos autovalores de $\left(r_{\star}, w_{\star}\right)_{1}$,

$$
\nu_{1,2}=\sqrt[4]{\tilde{C}^{2}-\frac{4 \tilde{D}}{3}}= \pm \sqrt{\tilde{C}} \sqrt[4]{1-\frac{4 \tilde{D}}{3 \tilde{C}^{2}}}
$$

Como os autovalores são compostos por funções do tipo $x^{1 / n}$ com $n$ par, então para órbitas críticas $D_{c}$, ocorre uma bifurcação de troca de estabilidade em $C_{c}$, onde surge um autovalor complexo puramente imaginário. No plano orbital, esta bifurcação é classificada como e Andronov-Hopf segundo a definição 2.32. Ainda no plano orbital, a órbita homoclínica para geodésicas do tipo luz representa uma trajetória que parte da fotosfera cujo o raio decresce até atingir um valor mínimo, onde volta a crescer tendendo assintoticamente à fotosfera, veja a esquerda da figura 25. No cenário crítico onde $D_{c}$ e $C_{c}$, temos a coalescência de ambas as fotosferas. Assim, toda a região interior a fotosfera $r=3 M$ já não é mais acessível uma vez que ela não está mapeada pelo sistema de coordenadas $\{t, r, \theta, \phi\}$, isso implica a destruição da órbita homoclínica. 

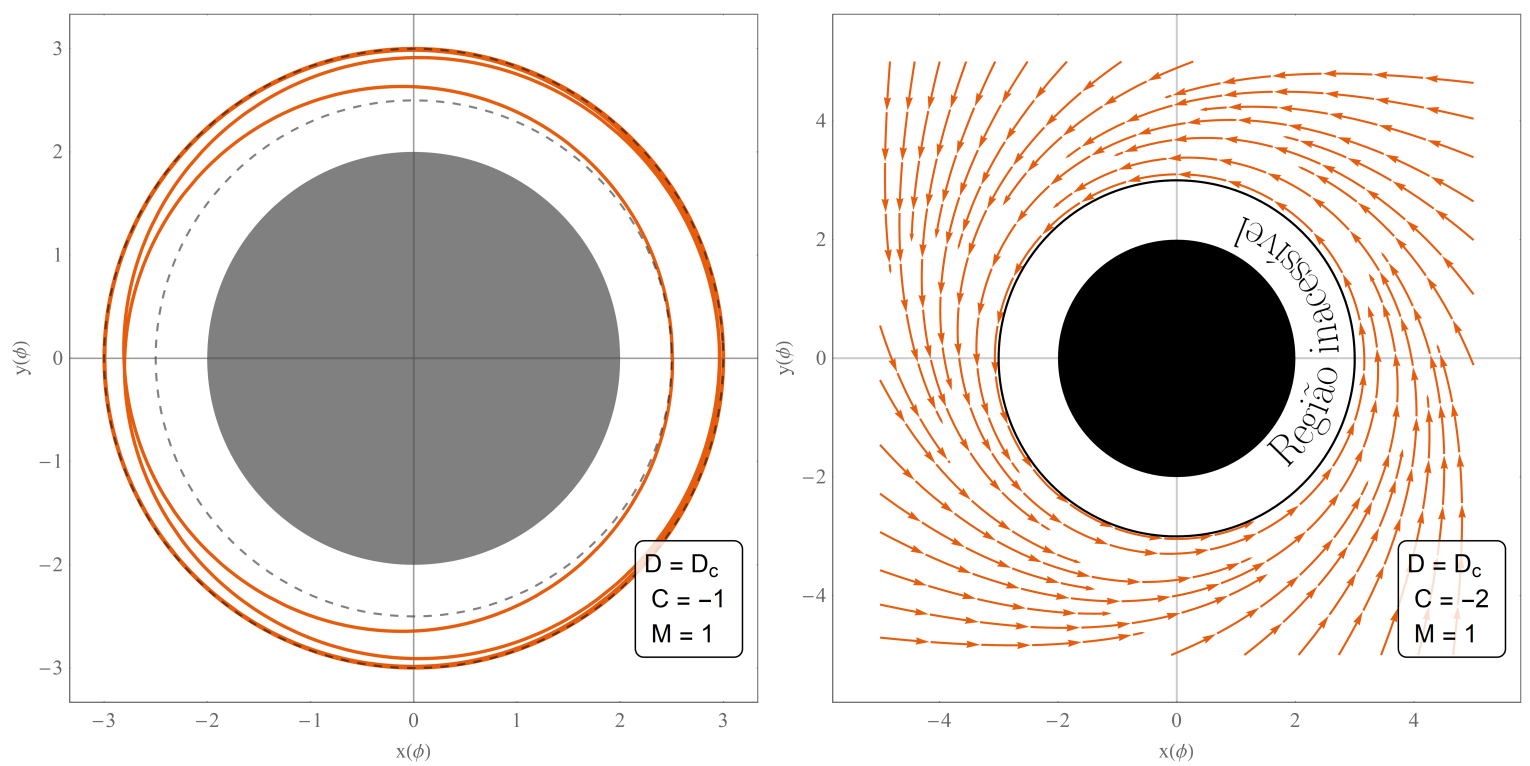

Figura 25 - Órbitas críticas de geodésica do tipo luz para $D_{c}=3 \sqrt{3} M$ : órbita homoclínica (esquerda) e região inacessível (direita).

Fonte: Elaborado pelo autor.

A bifurcação Andronov-Hopf daria origem a uma fotosfera estável em $r=3 M$, mas como já discutido, esta região não está mapeada. Assim, em toda região mapeada a fotosfera é instável. Com as bifurcações apresentadas acima, temos a descrição completa das geodésicas do tipo luz no contexto da geometria CFM.

É possível traçar um paralelo entre a estrutura do espaço-tempo e a dinâmica geodésica a partir das considerações deste capítulo. Como foi observado, a variação de $C$ altera a natureza da geometria, essa mudança pode ser observada a partir das geodésicas.

Nos casos de buracos negros com singularidades internas e horizontes simples $C>1$ ocorre a existência de uma única instável em $r=3 M$, enquanto que para o caso de um buraco negro sem singularidade $0 \leq C<1$, ainda existe apenas uma fotosfera instável em $r=3 M$, no entanto, observa-se uma órbita homoclínica no sistema. Os casos onde há um buraco de minhoca $C<0$, temos a emergência de uma fotosfera estável e acessível na garganta do buraco de minhoca, com a existência da órbita homoclínica até $C=-2$, quando já não há mais órbita estável, restando a única fotosfera instável na garganta do buraco de minhoca.

A título de verificação, é possível plotar a forma explícita do potencial efetivo associado ao buraco de minhoca ao realizar numericamente a mudança da variável radial para a coordenada quase-global $u$ e ainda realizar a extensão maximal desta coordenada, o que nos fornece a seguinte figura 26 . 

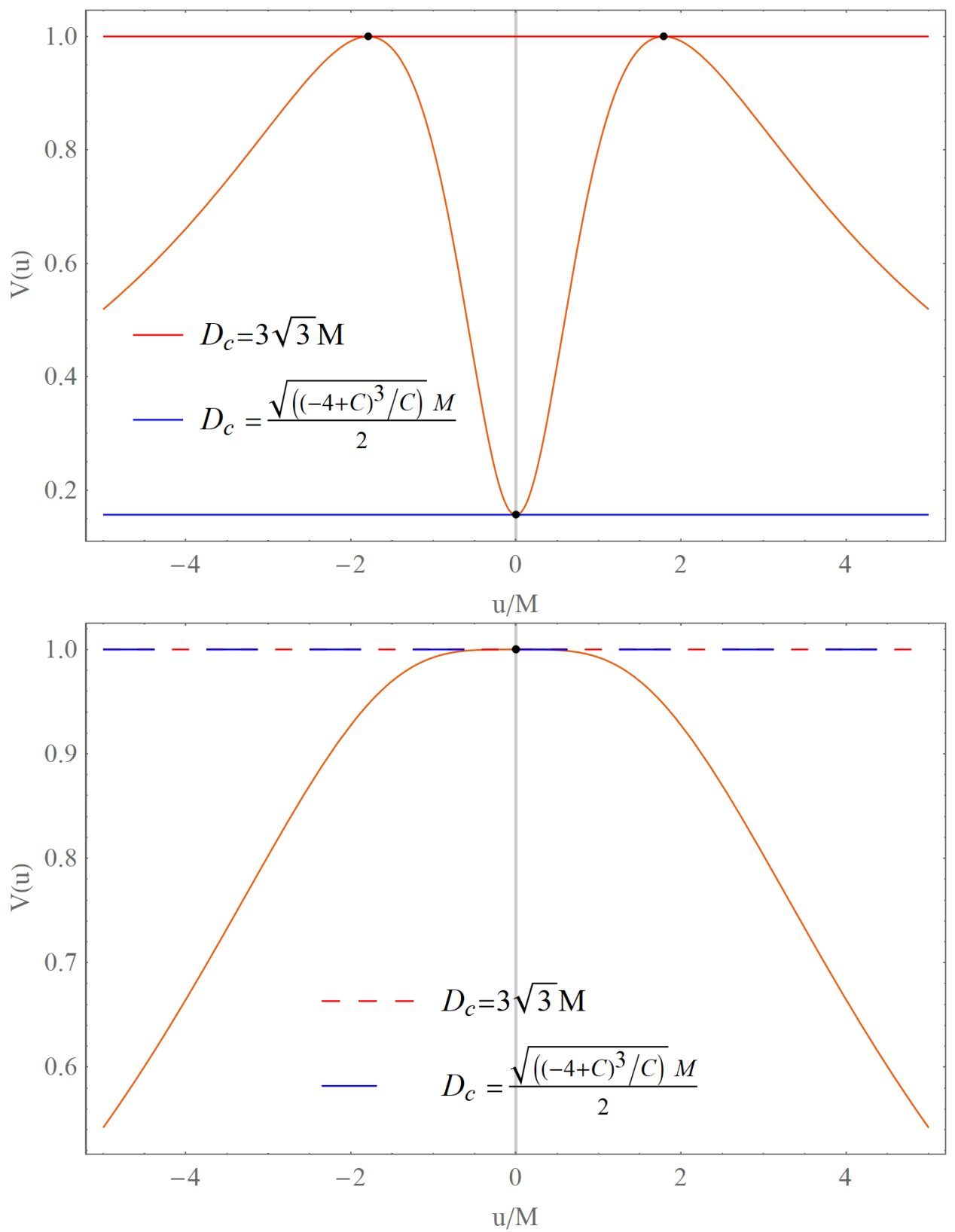

Figura 26 - Gráfico do potencial efetivo do buraco de minhoca CFM com $C=-0.1$ na figura de cima e $C=-2$ na figura de baixo.

Fonte: Elaborado pelo autor.

Pelas curvas do potencial efetivo, é possível ver que de fato a garganta do buraco de minhoca em $u=0$ é uma fotosfera estável pois se encontra em um mínimo local do potencial e a fotosferas em $u= \pm 1.79165 M$ (fotosfera em $r=3 M$ ) são instáveis pois estão em máximos locais do potencial, enquanto no caso crítico há apenas uma fotosfera instável na garganta $u=0$, confirmando a previsão realizada no sistema de coordenadas $r$. Olhando para a figura 26, podemos observar que mesmo no sistema de coordenadas $u$ o sistema é estruturalmente instável uma vez que que existe a possibilidade de formação de trajetórias heteroclínicas entre ambas as fotosferas instáveis. 


\section{COMENTÁRIOS FINAIS}

A análise de estabilidade de soluções de equações diferenciais ordinárias via teoria qualitativa tem se mostrado um poderoso arcabouço teórico para a compreensão de sistemas dinâmicos relativísticos na última década (KOHLI; HASLAM, 2018; BELBRUNO; PRETORIUS, 2011; ABOLGHASEM, 2013; ABOLGHASEM, 2012). A aplicação de diferentes técnicas e métodos se torna relevante a fim de se obter informações a respeito da robustez do sistema.

Através dos métodos de Lyapunov e Jacobi, pudemos observar um rico comportamento qualitativo. Inicialmente, as definições de estabilidade de Jacobi e Lyapunov foram comparadas para o contexto das geodésicas em geometrias com simetria esférica, onde foi verificado que ambas estão de fato em concordância. Em seguida, como uma geometria protótipo, foi analisada a geometria de Schwarzschild, onde a fotosfera foi caracterizada como um ponto de sela no retrato de fases do sistema e como um ciclo limite no plano orbital. Ainda no contexto de geometrias esféricas, foi mostrado que os centros no retrato de fases do sistema são robustos, através da construção de uma função de Lyapunov e indicando que este ponto é o seu mínimo local. A primeira geometria com constante cosmológica que estudamos a estabilidade da fotosfera foi para um buraco de minhoca de Sitter, onde mostramos que a garganta é uma fotosfera e, para o caso quase extremo, a sua estabilidade não muda ao variar os parâmetros $\Lambda$ e $C$. Ainda nesta geometria, a variação do parâmetro de impacto origina uma bifurcação do tipo fold de ciclos limites, variando a quantidade de pontos fixos do sistema, e aniquilando geodésicas que tendem à fotosfera.

Uma segunda geometria de interesse surgiu no contexto de mundos brana. A geometria CFM inicialmente proposta por (CASADIO; FABBRI; MAZZACURATI, 2002) representa uma solução com simetria esférica sem constante cosmológica. No entanto, na notação que adotamos aqui proposta por Molina e Neves (2012b), temos soluções parametrizadas por um parâmetro $C$ que nos fornece buracos negros ou de minhoca assintoticamente de Sitter.

A análise dinâmica deste espaço-tempo para as geodésicas do tipo luz se mostrou rica em termos de bifurcações. Para os casos de buracos negros regulares, a dinâmica é semelhante a de Schwarzschild, no entanto, para o caso do buraco de minhoca uma nova fotosfera estável, agora inerente à geometria e localizada na garganta do buraco, 
aparece na dinâmica e colide com a fotosfera instável, alterando a sua estabilidade após a bifurcação Bogdanov-Takens. Para avaliar a estabilidade da fotosfera na bifurcação, os coeficientes da forma normal da bifurcação foram avaliados, o que mostrou a ocorrência de uma bifurcação Bogndanov-Takens degenerada, cujo ponto de bifurcação é um cúspide instável. Foi observado também que este sistema permite a existência de uma órbita homoclínica que é a separatriz da região do sistema que permite estados ligados, isto é, geodésicas restritas a uma região limitada do plano orbital, com a região de espalhamento, sendo que após a BBT ocorre a destruição das órbitas estáveis.

As diferentes características dinâmicas das geodésicas entre os buracos negros sugerem que é possível inferir a estrutura do espaço-tempo interior ao horizonte através das geodésicas externas ao horizonte. Por exemplo, o buraco negro sem singularidade interna possui órbita do tipo luz homoclínica, enquanto os buracos negros singulares não a possuem. Uma outra característica, agora relacionada ao buraco de minhoca é a existência de uma fotosfera estável, atrapada por uma órbita homoclínica. Tais características podem ser indicadores da natureza do objeto ultracompacto observado observado, ou seja, a observação das propriedades associadas às geodésicas do tipo luz podem revelar a natureza interior aos horizontes de eventos, ou eventualmente distinguir os objetos entre buracos negros e buracos de minhoca.

No contexto da estabilidade de geodésicas, o uso dos coeficientes de Lyapunov deve ser realizado com cautela, pois estes não apresentam uma invariância em relação à mudanças de coordenadas, como mostrado por (CORNISH; LEVIN, 2003). Um estudo aprofundado sobre a covariância dos coeficientes de Lyapunov no contexto da relatividade geral seria uma possível extensão deste trabalho. Embora já existam trabalhos nesta direção (SZYDLOWSKI, 1993), estudos complementares a respeito do assunto se fazem necessários. 


\section{REFERÊNCIAS}

ABBOTT, B. P.; AL. et. Observation of gravitational waves from a binary black hole merger. Phys. Rev. Lett., American Physical Society, v. 116, p. 061102, Feb 2016. Citado na página 18.

ABOLGHASEM, H. Liapunov stability versus Jacobi stability. Journal of Dynamical Systems and Geometric Theories, Taylor \& Francis, v. 10, n. 1, p. 13-32, 2012. Citado 4 vezes nas páginas 34, 40, 61 e 97.

ABOLGHASEM, H. Stability of circular orbits in Schwarzschild spacetime. International Journal of Differential Equations and Applications, v. 12, n. 3, 2013. Citado 5 vezes nas páginas 35, 40, 41, 64 e 97.

ABRAHAM, R.; MARSDEN, J. E.; RATIU, T.; CUSHMAN, R. Foundations of mechanics. [S.l.]: Benjamin/Cummings Pub. Co, 1980. Citado na página 29.

AKIYAMA, K.; ALBERDI, A.; ALEF, W.; ASADA, K.; AZULAY, R.; BACZKO, A.-K.; BALL, D.; BALOKOVIĆ, M.; BARRETT, J.; BINTLEY, D. et al. First M87 event horizon telescope results. ii. array and instrumentation. The Astrophysical Journal Letters, IOP Publishing, v. 875, n. 1, p. L2, 2019. Citado na página 19.

AKIYAMA, K.; ALBERDI, A.; ALEF, W.; ASADA, K.; AZULAY, R.; BACZKO, A.-K.; BALL, D.; BALOKOVIĆ, M.; BARRETT, J.; BINTLEY, D. et al. First M87 event horizon telescope results. iii. data processing and calibration. The Astrophysical Journal Letters, IOP Publishing, v. 875, n. 1, p. L3, 2019. Citado na página 19.

AKIYAMA, K.; ALBERDI, A.; ALEF, W.; ASADA, K.; AZULAY, R.; BACZKO, A.-K.; BALL, D.; BALOKOVIĆ, M.; BARRETT, J.; BINTLEY, D. et al. First M87 event horizon telescope results. iv. imaging the central supermassive black hole. The Astrophysical Journal Letters, IOP Publishing, v. 875, n. 1, p. L4, 2019. Citado na página 19.

AKIYAMA, K.; ALBERDI, A.; ALEF, W.; ASADA, K.; AZULAY, R.; BACZKO, A.-K.; BALL, D.; BALOKOVIĆ, M.; BARRETT, J.; BINTLEY, D. et al. First M87 event horizon telescope results. v. physical origin of the asymmetric ring. The Astrophysical Journal Letters, IOP Publishing, v. 875, n. 1, p. L5, 2019. Citado na página 19.

AKIYAMA, K.; ALBERDI, A.; ALEF, W.; ASADA, K.; AZULAY, R.; BACZKO, A.-K.; BALL, D.; BALOKOVIĆ, M.; BARRETT, J.; BINTLEY, D. et al. First M87 event horizon telescope results. vi. the shadow and mass of the central black hole. The Astrophysical Journal Letters, IOP Publishing, v. 875, n. 1, p. L6, 2019. Citado na página 19.

ARNOL'D, V. I. Mathematical methods of classical mechanics. [S.1.]: Springer Science \& Business Media, 2013. v. 60. Citado 2 vezes nas páginas 29 e 30.

BARREIRA, L.; VALLS, C. Equações diferenciais ordinárias: teoria qualitativa. São Paulo: Livraria da Fúsica, 2012. Citado na página 30.

BELBRUNO, E.; PRETORIUS, F. A dynamical system's approach to Schwarzschild null geodesics. Classical and Quantum Gravity, v. 28, n. 19, p. 195007, 2011. Disponível em: 
〈http://stacks.iop.org/0264-9381/28/i=19/a=195007〉. Citado 2 vezes nas páginas 18 e 97.

BERNARDO, M.; BUDD, C.; CHAMPNEYS, A. R.; KOWALCZYK, P. Piecewise-smooth dynamical systems: theory and applications. [S.1.]: Springer Science \& Business Media, 2008. v. 163. Citado na página 25.

BOCCARA, N. Modeling complex systems. [S.l.]: Springer Science \& Business Media, 2010. Citado na página 19.

BOEHMER, C. G.; HARKO, T.; SABAU, S. et al. Jacobi stability analysis of dynamical systems: applications in gravitation and cosmology. Advances in Theoretical and Mathematical Physics, International Press of Boston, v. 16, n. 4, p. 1145-1196, 2012. Citado 3 vezes nas páginas 36, 40 e 41.

BOGDANOV, R. I. Versal deformations of a singular point of a vector field on the plane in the case of zero eigenvalues. Functional analysis and its applications, Springer, v. 9, n. 2, p. 144-145, 1975. Citado na página 44.

BRONNIKOV, K.; ELIZALDE, E.; ODINTSOV, S.; ZASLAVSKII, O. Horizons versus singularities in spherically symmetric space-times. Physical Review D, APS, v. 78, n. 6, p. 064049, 2008. Citado na página 58.

CARDOSO, V.; MIRANDA, A. S.; BERTI, E.; WITEK, H.; ZANCHIN, V. T. Geodesic stability, Lyapunov exponents, and quasinormal modes. Physical Review D, APS, v. 79, n. 6, p. 064016, 2009. Citado 6 vezes nas páginas 18, 20, 59, 69, 79 e 82.

CARROLL, S. Lectures notes on general relativity. [S.l.]: 〈https://ned.ipac.caltech.edu/ level5/March01/Carroll3/Carroll5.html), 2018. Citado na página 50.

CARROLL, S. M. Spacetime and Geometry: An Introduction to General Relativity. [S.1.]: Addison Wesley, 2004. ISBN 9780805387322. Citado 3 vezes nas páginas 47, 49 e 51.

CASADIO, R.; FABBRI, A.; MAZZACURATI, L. New black holes in the brane world? Physical Review D, APS, v. 65, n. 8, p. 084040, 2002. Citado 2 vezes nas páginas 59 e 97.

CATTANI, M. Dedução das equações da teoria de gravitação de einstein em um curso de graduação. Revista Brasileira de Ensino de Física, v. 20, p. 27-37, 1998. Citado na página 48.

CHANDRASEKHAR, S. The mathematical theory of black holes. [S.l.]: Oxford university press, 1998. v. 69. Citado 6 vezes nas páginas 51, 52, 63, 69, 71 e 74.

COLlABORATION, E. H. T. et al. First M87 event horizon telescope results. i. the shadow of the supermassive black hole. Astrophys. J. Lett, v. 875, p. L1, 2019. Citado na página 19.

CORNISH, N. J.; LEVIN, J. Lyapunov timescales and black hole binaries. Classical and Quantum Gravity, IOP Publishing, v. 20, n. 9, p. 1649, 2003. Citado 6 vezes nas páginas 18, 20, 63, 69, 70 e 98 .

DAVIDSON, M. C. Ix. a determination of the deflection of light by the sun's gravitational field, from observations made at the total eclipse of may 29, 1919. Phil. Trans. R. Soc. Lond. A, The Royal Society, v. 220, n. 571-581, p. 291-333, 1920. Citado na página 18. 
DÉCANINI, Y.; FOLACCI, A.; RAFFAELLI, B. Unstable circular null geodesics of static spherically symmetric black holes, regge poles, and quasinormal frequencies. Physical Review D, APS, v. 81, n. 10, p. 104039, 2010. Citado na página 53.

DEVANEY, M. W. H. R. Differential equations, dynamical systems, and an introduction to chaos. 2nd ed. ed. [S.1.]: Academic Press, 2004. (Pure and applied mathematics a series of monographs and textbooks 60). ISBN 9780080491141,9780123497031,0123497035. Citado na página 23.

DOERING, C. I.; LOPES, A. O. Equações diferenciais ordinárias. [S.l.]: Academic Press, 2008. (Coleção Matemática Universitária - IMPA, 517.2 DOE). Citado na página 75.

EICHHORN, R.; LINZ, S. J.; HÄNGGI, P. Transformation invariance of lyapunov exponents. Chaos, Solitons $\&$ Fractals, Elsevier, v. 12, n. 8, p. 1377-1383, 2001. Citado na página 20.

EINSTEIN, A. Die grundlage der allgemeinen relativitätstheorie. Annalen der Physik, Wiley Online Library, v. 354, n. 7, p. 769-822, 1916. Citado 2 vezes nas páginas 18 e 48.

GIUNTI, M.; MAZZOLA, C. Dynamical systems on monoids: Toward a general theory of deterministic systems and motion. Methods, models, simulations and approaches towards a general theory of change, p. 173-185, 2012. Citado 2 vezes nas páginas 21 e 23.

GREINER, W. Classical Mechanics: Systems of particles and Hamiltonian Dynamics. [S.l.]: Springer Science \& Business Media, 2003. Citado 4 vezes nas páginas 21, 22, 30 e 31.

HALE, J. K.; KOÇAK, H. Dynamics and bifurcations. [S.l.]: Springer Science \& Business Media, 2012. v. 3. Citado 2 vezes nas páginas 43 e 90.

HIRSCH, M. W.; SMALE, S.; DEVANEY, R. L. Differential equations, dynamical systems, and an introduction to chaos. [S.1.]: Academic press, 2012. Citado na página 22.

HOD, S. Fastest way to circle a black hole. Physical Review D, American Physical Society, v. 84, n. 10, p. 104024, 2011. Citado 2 vezes nas páginas 79 e 82.

HOMBURG, A. J.; SANDSTEDE, B. Homoclinic and heteroclinic bifurcations in vector fields. Handbook of dynamical systems, North-Holland Amsterdam, v. 3, p. 379-524, 2010. Citado 2 vezes nas páginas 24 e 25.

KIM, Y.; OH, C. Y.; PARK, N. Classical geometry of de sitter spacetime: An introductory review. arXiv preprint hep-th/0212326, 2002. Citado na página 56.

KOHLI, I. S.; HASLAM, M. C. Einstein's field equations as a fold bifurcation. Journal of Geometry and Physics, Elsevier, v. 123, p. 434-437, 2018. Citado na página 97.

KUZNETSOV, Y. A. Practical computation of normal forms on center manifolds at degenerate bogdanov-takens bifurcations. International Journal of Bifurcation and Chaos, World Scientific, v. 15, n. 11, p. 3535-3546, 2005. Citado 3 vezes nas páginas 43, 44 e 104.

KUZNETSOV, Y. A. Elements of applied bifurcation theory. [S.1.]: Springer Science \& Business Media, 2013. v. 112. Citado 5 vezes nas páginas 41, 42, 43, 44 e 86. 
LEE, J. Introduction to topological manifolds. [S.1.]: Springer Science \& Business Media, 2010. v. 940. Citado na página 23.

LEVIN, J.; O'REILLY, R.; COPELAND, E. J. Gravity waves from homoclinic orbits of compact binaries. Phys. Rev. D, American Physical Society, v. 62, p. 024023, Jun 2000. Citado na página 18.

LORENZ, E. N. Deterministic nonperiodic flow. Journal of the atmospheric sciences, v. 20, n. 2, p. 130-141, 1963. Citado 2 vezes nas páginas 26 e 27.

MISNER, C. W.; THORNE, K. S.; WHEELER, J. A. Gravitation. [S.l.]: Princeton University Press, 2017. Citado 5 vezes nas páginas 45, 47, 49, 53 e 67.

MOLINA, C.; NEVES, J. C. S. Wormholes in de sitter branes. Physical Review D, APS, v. 86, n. 2, p. 024015, 2012. Citado 2 vezes nas páginas 57 e 58.

MOLINA, C.; NEVES, J. C. S. Wormholes in de Sitter branes. Phys. Rev., D86, p. 024015, 2012. Citado na página 97.

MONTEIRO, L. H. A. Sistemas dinâmicos. [S.l.]: Editora Livraria da Física, 2006. Citado 7 vezes nas páginas $22,23,25,26,27,28$ e 29.

MUNKRES, J. R. Topology. [S.1.]: Prentice Hall, 2000. Citado na página 45.

O'NEILL, B. Semi-Riemannian geometry with applications to relativity. [S.l.]: Academic press, 1983. v. 103. Citado na página 45.

SABAU, S. Some remarks on Jacobi stability. Nonlinear Analysis: Theory, Methods 86 Applications, Elsevier, v. 63, n. 5-7, p. e143-e153, 2005. Citado 3 vezes nas páginas 20, 36 e 41.

SAYAMA, H. Introduction to the modeling and analysis of complex systems. [S.l.]: Open SUNY Textbooks, 2015. Citado 3 vezes nas páginas 19, 21 e 22.

SCHUTZ, B. F.; WILL, C. M. Black hole normal modes: a semianalytic approach. Astrophys.J., v. 291, p. L33-L36, 1985. Citado na página 55.

SHOOM, A. A. Metamorphoses of a photon sphere. Physical Review D, APS, v. 96, n. 8, p. 084056, 2017. Citado na página 18.

STROGATZ, S. H. Nonlinear dynamics and chaos: with applications to physics, biology, chemistry, and engineering. [S.l.]: CRC Press, 2018. Citado 4 vezes nas páginas 27, 75, 84 e 86.

SUHENDRO, I. Biography of Karl Schwarzschild (1873-1916). The Abraham Zelmanov Journal, v. 1, p. 14-19, 2008. Citado na página 54.

SZYDLOWSKI, M. Toward an invariant measure of chaotic behaviour in general relativity. Physics Letters A, Elsevier, v. 176, n. 1-2, p. 22-32, 1993. Citado 2 vezes nas páginas 20 e 98.

TAKENS, F. Unfoldings of certain singularities of vectorfields: Generalized hopf bifurcations. Journal of Differential Equations, Academic Press, v. 14, n. 3, p. 476-493, 1973. Citado na página 44. 
TAYLOR, J. R. Classical mechanics. [S.l.]: University Science Books, 2005. Citado na página 31.

VENEGEROLES, R. Integrabilidade em Sistemas Gravitacionais Axissimétricos. Dissertação (Mestrado) — Universidade de São Paulo, São Paulo, 1999. Citado 2 vezes nas páginas 33 e 34 .

VULPIANI, A. Chaos: from simple models to complex systems. [S.l.]: World Scientific, 2010. v. 17. Citado 4 vezes nas páginas 23, 26, 28 e 29.

WEINBERG, S.; DICKE, R. H. Gravitation and cosmology: principles and applications of the general theory of relativity. American Journal of Physics, American Association of Physics Teachers, v. 41, n. 4, p. 598-599, 1973. Citado 4 vezes nas páginas 46, 49, 51 e 55.

WILL, C. M. The confrontation between general relativity and experiment. Living Reviews in Relativity, Springer, v. 17, n. 1, p. 4, 2014. Citado na página 18.

WRESZINSKI, W. F. Mecânica clássica moderna. [S.l.]: Edusp, 1997. Citado na página 34. 


\section{APÊNDICE A - CÁLCULO DOS COEFICIENTES DA FORMA NORMAL DA BIFURCAÇÃO BOGDANOV-TAKENS}

Neste apêndice obteremos os coeficientes da forma normal associada à bifurcação de Bogdanov-Takens, utilizados na seção 4.6. No trabalho de Kuznetsov (2005) foi sugerido um método prático para encontrar os coeficientes da forma normal do sistema baseado na alternativa de Fredholm, fornecendo coeficientes até de quarta ordem da forma normal da Bogdanov-Takens. Considere o seguinte sistema de EDOs

$$
\dot{x}=A x+F(x), x \in \mathbb{R}^{n},
$$

com $F(x)$ suave. A sua expansão em série de Taylor é dada por,

$$
F(x)=\frac{1}{2} B(x, x)+\frac{1}{3 !} C(x, x, x)+\frac{1}{4 !} D(x, x, x, x)+\mathcal{O}\left(\left\|x^{5}\right\|\right),
$$

onde

$$
\begin{gathered}
B_{i}(x, y)=\left.\sum_{j, k=1}^{n} \frac{\partial^{2} F_{i}(\xi)}{\partial \xi_{j} \partial \xi_{k}}\right|_{\xi=0} x_{j} y_{k}, \\
C_{i}(x, y, z)=\left.\sum_{j, k, l=1}^{n} \frac{\partial^{3} F_{i}(\xi)}{\partial \xi_{j} \partial \xi_{k} \partial \xi_{l}}\right|_{\xi=0} x_{j} y_{k} z_{l}, \\
D_{i}(x, y, z, v)=\left.\sum_{j, k, l, m=1}^{n} \frac{\partial^{4} F_{i}(\xi)}{\partial \xi_{j} \partial \xi_{k} \partial \xi_{l} \partial \xi_{m}}\right|_{\xi=0} x_{j} y_{k} z_{l} v_{m},
\end{gathered}
$$

para $i=1,2, \ldots, n$.

Os autovalores $q_{0}, q_{1}$ na bifurcação são os autovalores generalizados da matriz Jacobiana do sistema e $p_{0}, p_{1}$ os autovalores da Jacobiana transposta. Estes vetores devem ser tais que,

$$
\left\langle p_{0}, q_{0}\right\rangle\left\langle p_{1}, q_{1}\right\rangle=1,\left\langle p_{0}, q_{1}\right\rangle=\left\langle p_{1}, q_{0}\right\rangle=0
$$

Com estas quantidades, é possível avaliar os coeficientes quadráticos que são dados pelas seguintes expressões,

$$
\begin{gathered}
a_{2}=\frac{1}{2}\left\langle p_{1}, B\left(q_{0}, q_{0}\right)\right\rangle, \\
b_{2}=\left\langle p_{1}, B\left(q_{0}, q_{1}\right)\right\rangle-\left\langle p_{1}, h_{20}\right\rangle=\left\langle p_{1}, B\left(q_{0}, q_{1}\right)\right\rangle+\left\langle p_{0}, B\left(q_{0}, q_{0}\right)\right\rangle,
\end{gathered}
$$

onde $h_{20}$ é o vetor solução do seguinte sistema,

$$
A h_{20}=2 a_{2} q_{1}-B\left(q_{0}, q_{0}\right)
$$


Os coeficientes cúbicos são dados por,

$$
a_{3}=\frac{1}{6}\left\langle p_{1}, C\left(q_{0}, q_{0}, q_{0}\right)\right\rangle+\frac{1}{2}\left\langle p_{1}, B\left(h_{20}, q_{0}\right)\right\rangle-a_{2}\left\langle p_{1}, h_{11}\right\rangle
$$

onde $h_{11}$ é o vetor solução do seguinte sistema

$$
A h_{11}=b_{2} q_{1}+h_{20}-B\left(q_{0}, q_{1}\right)
$$

e o coeficiente $b_{3}$,

$$
b_{3}=\frac{1}{2}\left\langle p_{1}, C\left(q_{0}, q_{0}, q_{1}\right)+2 B\left(h_{11}, q_{0}\right)+B\left(h_{20}, q_{1}\right)\right\rangle-\frac{1}{2}\left\langle p_{1}, h_{30}+2 a_{2} h_{02}+2 b_{2} h_{11}\right\rangle
$$

com os vetores $h_{02}, h_{30}$ solução dos respectivos sistemas,

$$
\begin{aligned}
& A h_{02}=2 h_{11}-B\left(q_{1}, q_{1}\right) \\
& A h_{30}=6 q_{1} a_{3}+6 h_{11} a_{2}-3 B\left(h_{20}, q_{0}\right)-C\left(q_{0}, q_{0}, q_{0}\right) .
\end{aligned}
$$

Finalmente, os termos de quarta ordem são fornecidos pelas seguintes expressões

$$
\begin{gathered}
a_{4}=\frac{1}{24}\left\langle p_{1}, D\left(q_{0}, q_{0}, q_{0}, q_{0}\right)+6 C\left(h_{20}, q_{0}, q_{0}\right)+\right. \\
\left.4 B\left(h_{30}, q_{0}\right)+3 B\left(h_{20}, h_{20}\right)\right\rangle-\frac{1}{2} a_{2}\left\langle p_{1}, h_{11}\right\rangle, \\
b_{4}=\frac{1}{6}\left\langle p_{1}, D\left(q_{0}, q_{0}, q_{0}, q_{1}\right)+3 C\left(h_{20}, q_{0}, q_{1}\right)+3 C\left(h_{11}, q_{0}, q_{0}\right)\right\rangle+ \\
\frac{1}{6}\left\langle p_{1}, 3 B\left(h_{21}, q_{0}\right)+B\left(h_{11}, h_{20}\right)+B\left(h_{30}, q_{1}\right)\right\rangle-\frac{1}{6}\left\langle p_{1}, h_{40}\right\rangle \\
-\frac{1}{2} b_{2}\left\langle p_{1}, h_{21}\right\rangle-\left\langle p_{1}, a_{2} h_{12}+a_{3} h_{02}+b_{3} h_{11}\right\rangle,
\end{gathered}
$$

com os vetores $h_{30}, h_{21}$ e $h_{40}$ satisfazendo o sistema,

$$
\begin{gathered}
A h_{20}=6 q_{1} a_{3}+6 h_{11} a_{2}-3 B\left(h_{20}, q_{0}\right)-C\left(q_{0}, q_{0}, q_{0}\right) \\
A h_{21}=h_{30}+2 b_{3} q_{1}+2 a_{2} h_{02}+2 b_{2} h_{11}-2 B\left(h_{11}, q_{0}\right)-B\left(h_{20}, q_{1}\right)-C\left(q_{0}, q_{0}, q_{1}\right), \\
A h_{40}=24 a_{4} q_{1}+12 a_{2} h_{21}+24 a_{3} h_{11}-4 B\left(h_{30}, q_{0}\right) \\
+3 B\left(h_{20}, h_{20}\right)-6 C\left(h_{20}, q_{0}, q_{0}\right)-D\left(q_{0}, q_{0}, q_{0}, q_{0}\right) .
\end{gathered}
$$

Uma vez definidas as expressões dos coeficientes, avaliamos a matriz Jacobiana no ponto de bifurcação e obtemos a exatamente a matriz,

$$
A=\left(\begin{array}{ll}
0 & 1 \\
0 & 0
\end{array}\right),
$$


cujos autovetores generalizados são,

$$
q_{0}=(1,0)^{T}, \quad q_{1}=(0,1)^{T}
$$

e os autovetores generalizados da sua transposta,

$$
p_{1}=(0,1)^{T}, \quad p_{0}=(1,0)^{T}
$$

satisfazendo as condições (278). Calcula-se também os coeficientes de $B(x, x)$,

$$
\begin{gathered}
\frac{\partial^{2} F_{1}}{\partial r^{2}}=\frac{\partial^{2} F_{1}}{\partial w \partial r}=\frac{\partial^{2} F_{1}}{\partial r \partial w}=\frac{\partial^{2} F_{1}}{\partial w^{2}}=0, \\
\frac{\partial^{2} F_{2}}{\partial r^{2}}=\frac{1}{2} \frac{\mathrm{d}^{3} f_{\mathrm{CFM}}}{\mathrm{d} r^{3}}, \quad \frac{\partial^{2} F_{2}}{\partial w \partial r}=\frac{\partial^{2} F_{2}}{\partial r \partial w}=\frac{\partial^{2} F_{2}}{\partial w^{2}}=0 .
\end{gathered}
$$

Aplicando os coeficientes não nulos de $B(x, x)$, temos,

$$
B\left(q_{0}, q_{0}\right)=\left(\begin{array}{c}
0 \\
2 / 3
\end{array}\right) \text {. }
$$

Podemos encontrar o termo quadrático $a_{2}$ através da equação (279),

$$
a_{2}=\frac{1}{2}\left(\begin{array}{l}
0 \\
1
\end{array}\right)\left(\begin{array}{c}
0 \\
2 / 3
\end{array}\right)=\frac{1}{3} .
$$

Avaliando o vetor $h_{20}$, temos que

$$
A h_{20}=\frac{2}{3}\left(\begin{array}{l}
0 \\
1
\end{array}\right)-\left(\begin{array}{c}
0 \\
2 / 3
\end{array}\right),
$$

cuja a solução é dada pelo vetor

$$
h_{20}=\left(\begin{array}{l}
\alpha \\
0
\end{array}\right), \quad \alpha \in \mathbb{R} .
$$

o coeficiente $B\left(q_{0}, q_{1}\right)$ fornece

$$
B\left(q_{0}, q_{1}\right)=\left(\begin{array}{l}
0 \\
0
\end{array}\right)
$$

assim, o coeficiente $b_{2}$ é encontrado:

$$
b_{2}=(0,1)(0,0)^{T}+(0,0)(0,2 / 3)^{T}=0 .
$$

De onde se observa que a bifurcação de Bogdanov-Takens que ocorre no sistema é degenerada, pois o coeficiente $b_{2}$ é zero. Neste cenário, a forma normal do sistema será 
dado pelo sistema (71), logo devemos encontrar o coeficiente $b_{4}$. Avaliando o vetor $h_{11}$ temos,

$$
A h_{11}=\left(\begin{array}{l}
\alpha \\
0
\end{array}\right)-\left(\begin{array}{l}
0 \\
0
\end{array}\right)=\left(\begin{array}{l}
\alpha \\
0
\end{array}\right)
$$

cuja solução é

$$
h_{11}=\left(\begin{array}{l}
\delta \\
\alpha
\end{array}\right), \quad \delta \in \mathbb{R}
$$

e com relação ao vetor $h_{02}$, temos

$$
A h_{02}=2\left(\begin{array}{l}
\delta \\
\alpha
\end{array}\right)
$$

de onde podemos concluir que $\alpha=0$ e a solução é dada por

$$
h_{02}=\left(\begin{array}{c}
\xi \\
2 \delta
\end{array}\right), \quad \xi \in \mathbb{R} .
$$

Os coeficientes de $C(u, v, w)$,

$$
\begin{gathered}
C_{1}(u, v, w)=0 \\
C_{2}(u, v, w)=\frac{1}{2} \frac{\mathrm{d}^{4} f_{\mathrm{CFM}}}{\mathrm{d} r^{4}}=-\frac{112}{27} u_{1} v_{1} w_{1},
\end{gathered}
$$

onde $u_{1}, v_{1}$ e $w_{1}$ são as componentes dos vetores $u, v, w$. Desta forma, temos os seguintes coeficientes:

$$
\begin{gathered}
C\left(q_{0}, q_{0}, q_{0}\right)=\left(\begin{array}{c}
0 \\
-112 / 27
\end{array}\right), \\
C\left(q_{0}, q_{0}, q_{1}\right)=B\left(q_{1}, q_{1}\right)=B\left(h_{12}, q_{1}\right)=B\left(h_{20}, q_{0}\right)=\left(\begin{array}{l}
0 \\
0
\end{array}\right), \\
B\left(h_{11}, q_{0}\right)=\left(\begin{array}{c}
0 \\
2 \delta / 3
\end{array}\right) .
\end{gathered}
$$

Com estes valores em mãos, calcula-se o coeficiente $a_{3}$ como segue,

$$
a_{3}=\frac{1}{6}\left(\begin{array}{l}
0 \\
1
\end{array}\right)\left(\begin{array}{c}
0 \\
-112 / 27
\end{array}\right)^{T}+\frac{1}{2}\left(\begin{array}{l}
0 \\
1
\end{array}\right)\left(\begin{array}{l}
0 \\
0
\end{array}\right)^{T}
$$

fornecendo,

$$
a_{3}=-\frac{1}{6} \cdot \frac{112}{27}
$$


Avaliando o vetor $h_{30}$, temos

$$
A h_{30}=-6\left(\begin{array}{l}
0 \\
1
\end{array}\right) \frac{112}{27}+2\left(\begin{array}{l}
\delta \\
0
\end{array}\right)-\left(\begin{array}{c}
0 \\
-112 / 27
\end{array}\right)
$$

cujo vetor solução $h_{30}$ é dado por

$$
h_{30}=\left(\begin{array}{c}
\sigma \\
2 \delta
\end{array}\right), \quad \sigma \in \mathbb{R}
$$

Calculando agora o coeficiente $b_{3}$,

$$
\begin{aligned}
b_{3}=\frac{1}{2}\left\langle p_{1},\right. & \underbrace{C\left(q_{0}, q_{0}, q_{1}\right)}_{=0}+2 B\left(h_{11}, q_{0}\right)+\underbrace{B\left(h_{20}, q_{1}\right)}_{=0}\rangle+ \\
& -\frac{1}{2}\left\langle p_{1}, h_{30}+2 a_{2} h_{02}+2 b_{2} h_{11}\right\rangle,
\end{aligned}
$$

assim resta apenas

$$
\begin{gathered}
b_{3}=\frac{1}{2}\left\langle p_{1}, 2 B\left(h_{11}, q_{0}\right)\right\rangle-\frac{1}{2}\left\langle p_{1}, h_{30}+2 a_{2} h_{02}+2 b_{2} h_{11}\right\rangle, \\
b_{3}=\frac{1}{2}\left(\begin{array}{l}
0 \\
1
\end{array}\right)\left(\begin{array}{c}
0 \\
4 \delta / 3
\end{array}\right)^{T}-\left(\begin{array}{l}
0 \\
1
\end{array}\right)\left(\begin{array}{c}
2 \xi+3 \sigma / 3 \\
10 \delta / 3
\end{array}\right)^{T}=\frac{2}{3} \delta-\frac{5}{3} \delta=-\delta,
\end{gathered}
$$

de onde vemos que $b_{3}$ assume o valor de uma contante livre. Sem perdas de generalidade, podemos escolher $\delta=0$ de forma que $b_{3}=0$. Com $\delta$ igual a zero os nossos vetores assumem o seguintes valores,

$$
h_{20}=h_{11}=\left(\begin{array}{l}
0 \\
0
\end{array}\right), \quad h_{02}=\left(\begin{array}{l}
\xi \\
0
\end{array}\right), \quad h_{30}=\left(\begin{array}{l}
\sigma \\
0
\end{array}\right) .
$$

Avaliando agora o vetor $h_{21}$, encontramos

$$
A h_{21}=\left(\begin{array}{l}
\sigma \\
0
\end{array}\right)+\frac{2}{3}\left(\begin{array}{l}
\xi \\
0
\end{array}\right)=\left(\begin{array}{c}
\sigma+2 \xi / 3 \\
0
\end{array}\right)
$$

assim, temos como solução o vetor $h_{21}$,

$$
h_{21}=\left(\begin{array}{c}
\Sigma \\
\sigma+2 \xi / 3
\end{array}\right), \quad \Sigma \in \mathbb{R} .
$$

Verifica-se que os coeficientes $C\left(q_{0}, q_{1}, q_{1}\right)$ e $B\left(h_{02}, q_{0}\right)$ são dados por

$$
C\left(q_{0}, q_{1}, q_{1}\right)=\left(\begin{array}{l}
0 \\
0
\end{array}\right)
$$


$\mathrm{e}$

$$
B\left(h_{02}, q_{0}\right)=\left(\begin{array}{c}
0 \\
2 \xi / 3
\end{array}\right)
$$

Agora, calculando o vetor $h_{12}$, temos a seguinte expressão,

$$
A h_{21}=2\left(\begin{array}{c}
\Sigma \\
\sigma+2 \xi / 3
\end{array}\right)-\left(\begin{array}{c}
0 \\
2 \xi / 3
\end{array}\right)=\left(\begin{array}{c}
2 \Sigma \\
2 \sigma+2 \xi / 3
\end{array}\right)
$$

de onde é possível concluir que $\sigma=-\xi / 3$ e que nos gera o vetor solução $h_{12}$

$$
h_{12}=\left(\begin{array}{c}
\theta \\
2 \Sigma
\end{array}\right), \quad \theta \in \mathbb{R}
$$

Portanto, temos o seguinte conjunto de vetores,

$$
\begin{gathered}
h_{20}=h_{11}=\left(\begin{array}{l}
0 \\
0
\end{array}\right), \quad h_{02}=\left(\begin{array}{l}
\xi \\
0
\end{array}\right), \quad h_{30}=\left(\begin{array}{c}
-\xi / 3 \\
0
\end{array}\right), \\
h_{21}=\left(\begin{array}{c}
\Sigma \\
\xi / 3
\end{array}\right), \quad h_{12}=\left(\begin{array}{c}
\theta \\
2 \Sigma
\end{array}\right) .
\end{gathered}
$$

Verifica-se também que os coeficientes $B\left(h_{02}, q_{1}\right)$ e $C\left(q_{1}, q_{1}, q_{1}\right)$ são

$$
B\left(h_{02}, q_{1}\right)=\left(\begin{array}{l}
0 \\
0
\end{array}\right), \quad C\left(q_{1}, q_{1}, q_{1}\right)=\left(\begin{array}{l}
0 \\
0
\end{array}\right) .
$$

Calculando o vetor $h_{03}$, obtemos

$$
A h_{03}=\left(\begin{array}{c}
2 \theta \\
6 \Sigma
\end{array}\right)
$$

de onde concluímos que $\Sigma=0$ e o vetor solução toma a forma

$$
h_{03}=\left(\begin{array}{c}
\psi \\
3 \theta
\end{array}\right), \quad \psi \in \mathbb{R}
$$

Com essas informações em mãos podemos perceber que a expressão (287) assume a seguinte forma,

$$
a_{4}=\frac{1}{24}\left\langle p_{1}, D\left(q_{0}, q_{0}, q_{0}, q_{0}\right)+4 B\left(h_{30}, q_{0}\right)\right\rangle-\frac{1}{2} a_{2}\left\langle p_{1}, h_{21}\right\rangle .
$$

Assim, avaliamos os coeficientes $D\left(q_{0}, q_{0}, q_{0}, q_{0}\right)$ e $C\left(h_{20}, q_{0}, q_{0}\right)$ :

$$
D\left(q_{0}, q_{0}, q_{0}, q_{0}\right)=\left(\begin{array}{c}
0 \\
\frac{\mathrm{d}^{5} f_{\mathrm{CFM}}}{\mathrm{d} r^{5}}
\end{array}\right)=\left(\begin{array}{c}
0 \\
1720 / 81
\end{array}\right)
$$




$$
C\left(h_{20}, q_{0}, q_{0}\right)=\left(\begin{array}{l}
0 \\
0
\end{array}\right), \quad B\left(h_{30}, q_{0}\right)=\left(\begin{array}{c}
0 \\
-2 \xi / 9
\end{array}\right) .
$$

Desta forma o coeficiente $a_{4}$ assume a seguinte forma,

$$
a_{4}=\frac{1}{24}\left\langle\left(\begin{array}{l}
0 \\
1
\end{array}\right),\left(\begin{array}{c}
0 \\
1720 / 81
\end{array}\right)+4\left(\begin{array}{c}
0 \\
-2 \xi / 9
\end{array}\right)\right\rangle-\frac{1}{6}\left\langle\left(\begin{array}{l}
0 \\
1
\end{array}\right),\left(\begin{array}{c}
0 \\
\xi / 3
\end{array}\right)\right\rangle
$$

produzindo o seguinte coeficiente $a_{4}$,

$$
a_{4}=-\frac{5}{486}(9 \xi-86)
$$

que novamente, depende de uma constante arbitrária. Escolhemos $\xi$ tal que

$$
9 \xi-86=0 \Leftrightarrow \xi=\frac{86}{9} .
$$

Finalmente o coeficiente $a_{4}$ é determinado, $a_{4}=0$, e os nossos vetores se tornam,

$$
\begin{gathered}
h_{20}=h_{11}=\left(\begin{array}{l}
0 \\
0
\end{array}\right), \quad h_{02}=\left(\begin{array}{c}
86 / 9 \\
0
\end{array}\right), \quad h_{30}=\left(\begin{array}{c}
-86 / 27 \\
0
\end{array}\right), \\
h_{21}=\left(\begin{array}{c}
0 \\
86 / 27
\end{array}\right), \quad h_{12}=\left(\begin{array}{l}
\theta \\
0
\end{array}\right), \quad h_{03}=\left(\begin{array}{c}
\psi \\
3 \theta
\end{array}\right) .
\end{gathered}
$$

A fim de calcular $b_{4}$, avaliamos os seguintes coeficientes,

$$
D\left(q_{0}, q_{0}, q_{0}, q_{1}\right)=B\left(h_{30}, q_{1}\right)=\left(\begin{array}{l}
0 \\
0
\end{array}\right)
$$

e calculando $h_{40}$,

$$
A h_{40}=12 a_{2} h_{21}+24 a_{3} h_{11}-4 B\left(h_{30}, q_{0}\right)-D\left(q_{0}, q_{0}, q_{0}, q_{0}\right)
$$

que nos conduz ao seguinte sistema,

$$
A h_{40}=\left(\begin{array}{l}
0 \\
0
\end{array}\right)
$$

com solução

$$
h_{40}=\left(\begin{array}{l}
k \\
0
\end{array}\right), \quad k \in \mathbb{R}
$$

Finalmente, avaliando o coeficiente de quarta ordem $b_{4}$,

$$
b_{4}=\frac{1}{6}\left(\begin{array}{l}
0 \\
1
\end{array}\right)\left(\begin{array}{l}
k \\
0
\end{array}\right)^{T}-\left(\begin{array}{l}
0 \\
1
\end{array}\right)\left(\begin{array}{c}
\frac{\theta}{2}-\frac{4816}{729} \\
0
\end{array}\right)^{T}=0
$$


Assim os coeficientes da forma normal do sistema são todos determinados. Os resultados obtidos são apresentados na tabela 1 a seguir.

\begin{tabular}{|c|c|}
\hline Coeficiente & Valor \\
\hline \hline$a_{2}$ & $1 / 3$ \\
$b_{2}$ & 0 \\
$a_{3}$ & $-56 / 81$ \\
$b_{3}$ & 0 \\
$a_{4}$ & 0 \\
$b_{4}$ & 0 \\
\hline
\end{tabular}

Tabela 1 - Coeficientes da forma normal da bifurcação Bogdanov-Takens.

Fonte: Elaborado pelo autor. 


\section{ÍNDICE REMISSIVO}

Gravitação, 45

símbolos de Christoffel, 47

campos de Killing, 50, 55, 56

delta de Kronecker, 46

derivada covariante, 47

equação

de campo de Einstein, 48

de Killing, 50

escalar de Ricci, 48

espaço-tempo, 45

geodésicas

tipo luz, 51

tipo tempo, 51

horizonte cosmológico, 56

Karl Schwarzschild, 54

métrica, 45

CFM, 59

de Minkowski, 56

de Schwarzschild, 55

de Sitter, 56

Lorentziana, 45

wh_dS, 57

parâmetro

afim, 51

de impacto, 67

tensor

de Ricci, 47

de Riemann-Christoffel, 47

energia-momento, 49

teorema de Birkhoff, 55 vetores

contravariantes, 46

covariantes, 46

Sistemas Dinâmicos, 21

órbita

heteroclínica, 24

homoclínica, 24

atrator, 25

bacia

de atração, 26

de repulsão, 26

bifurcação, 42

Andronov-Hopf, 42

fold, 42

Bogdanov-Takens, 43

centro-sela, 42

centros, 26

ciclo limite, 27

assintoticamente estável, 27

assintoticamente instável, 27

semi-estável, 27

conexão

de Berwald, 39

não-linear, 39

contínuos no tempo, 22

critério

de Bendixson, 27

do ponto fixo, 27

curvas isóclinas, 23

definição, 21 
equações de Hamilton, 30

equivalência topológica, 41

espaço de estados, 22

Floris Takens, 44

Hamiltonianos, 29

Jacobi

estabilidade de, 36, 40

$\mathrm{KCC}$

diferencial, 37

KCC-invariante

primeiro, 37

segundo, 39

Lyapunov

assintoticamente estável, 35

estabilidade de, 34

matriz Jacobiana, 33

pontos fixos, 23

hiperbólicos, 29

repulsor, 26

Rifkat Bognadov, 44

teorema de

estabilidade de Lyapunov, 35

Liouville, 31 